\title{
Catalog of the types of Curculionoidea (Insecta, Coleoptera) deposited at the Museo Argentino de Ciencias Naturales "Bernardino Rivadavia", Buenos Aires
}

\author{
Axel O. BACHMANN ${ }^{1} \&$ Analía A. LANTERI ${ }^{2}$ \\ ${ }^{1}$ Museo Argentino de Ciencias Naturales, División Entomología, Buenos Aires C1405DJR. Universidad de \\ Buenos Aires, Facultad de Ciencias Exactas y Naturales, Buenos Aires C1428EHA, e-mail: bachmann@bg.fcen. \\ uba.ar. ${ }^{2}$ Museo de La Plata, División Entomología, Paseo del Bosque s/n, La Plata, B1900FWA, Argentina, \\ e-mail: alanteri@fcnym.unlp.edu.ar
}

\begin{abstract}
The type specimens of Curculionoidea (Apionidae, Brentidae, Anhribidae, Curculionidae, Platypodidae, and Scolytidae) from the Museo Argentino de Ciencias Naturales (MACN), corresponding to all current categories, are herein catalogued. A total of 344 specific and subspecific names are alphabetically recorded, for their original binomina or trinomina, and spellings. Later combinations and synonyms are mentioned, as well as the information of all the labels associated to the specimens. In order to assist future research, three further lists are added: 1. specimens deemed to be deposited at MACN but not found in the collection; 2. specimens labeled as types of species which descriptions have probably never been published (non available names); and 3. specimens of dubious type status, because the information on the labels does not agree with that of the original publication.
\end{abstract}

Key words: Type specimens, Curculionoidea, Coleoptera, Insecta.

Resumen: Catálogo de los tipos de Curculionoidea (Insecta, Coleoptera) depositados en el Museo Argentino de Ciencias Naturales "Bernardino Rivadavia", Buenos Aires. Se catalogan los ejemplares tipo de Curculionoidea (Apionidae, Brentidae, Anhribidae, Curculionidae, Platypodidae y Scolytidae) depositados en el Museo Argentino de Ciencias Naturales (MACN), correspondientes a todas las categorías de tipos aceptadas. Se registran 344 nombres específicos y subespecíficos, que se ordenan alfabéticamente según sus binomios, o trinomios, y grafías originales. Se mencionan las combinaciones posteriores y los sinónimos conocidos, y se brinda una descripción detallada de todos los rótulos del material mencionado. Para facilitar futuras investigaciones se agregan otras tres listas: 1 . ejemplares tipo supuestamente depositados en el MACN pero no hallados en la colección; 2. ejemplares rotulados como tipos, pero cuyos nombres específicos o subespecíficos son no disponibles, pues sus descripciones probablemente no han sido publicadas; y 3. ejemplares rotulados como tipos pero cuyo status como tales es dudoso pues no hay concordancia entre los datos de los rótulos y los de la publicación.

Palabras clave: Ejemplares tipo, Curculionoidea, Coleoptera, Insecta.

\section{INTRODUCTION}

A catalog of the type specimens of Curculionoidea deposited at the Entomology Division of the Museo Argentino de Ciencias Naturales (MACN) is herein presented. It includes 344 specific and subspecific names in alphabetical order, according to their original spellings. A brief bibliographic quoting, the information content of all labels associated to each type, as well as later nomenclatural or taxonomical changes have been added.

The classification of Curculionoidea follows Lawrence \& Newton (1995) and AlonsoZarazaga \& Lyal (1999). Herein we record type specimens of the families Apionidae, Brentidae, Anthribidae, Curculionidae, Platypodidae and Scolytidae. The latter two families are probably part of Curculionidae (Marvaldi et al., 2002), however, we have considered them separately for practical purposes.

For checking available and valid names, synonymies and new combinations of the American Curculionidae and Apionidae we used the checklist of Wibmer \& O'Brien (1986, 1989b); for Scolytidae and Platypodidae, the catalogs of Wood \& Bright (1992), and Bright \& Skidmore (1997, 2002); for Anthribidae, the catalog of Rheinheimer (2004), and for Brentidae, Sforzi \& Bartolozzi (2004). 
Most species herein recorded belong to Curculionidae and were described by Alphonse Hustache (more than $150 \mathrm{spp}$ ) based on specimens sent to him by Carlos Bruch. Other authors that contributed with numerous types are G. Kuschel (36 spp), J. Brèthes (31 spp), K. Heller (22 spp), L. Béguin-Billecoq (17 spp) and M. J. Viana (16 spp).

For the specimens not formally designated in the original publication, evidences from the labels or from the specimens have been taken into account for considering them as types (art. 72.4 of the International Code of Zoological Nomenclature, 1999). When holotypes were not designated, or when the number of specimens of the type series was not stated, the specimens were considered syntypes (recommendation $73 \mathrm{~F}$ of the Code).

The specimens labeled as types but not found in the literature are not available in the sense of the Code, however, in order to assist future research we mention them in a separate list. Other two lists have been compiled for missing types (deemed to be housed at MACN but not found) and for dubious types (specimens labeled as types but not regarded as such, because the information on the labels does not agree with that of the original publication).

Hermann Burmeister did not label the type specimens of his collection, instead, he used to attach to the bottom of the drawer where the specimens were stored a label with the name of the species, including a vague geographical indication, such as "Rep. Argent." for Argentina, or "Patag." for Patagonia. This is why the type status of these specimens is so hard to be certified. Carlos Berg, the follower of Burmeister as the director of the Museum, used to attach a tiny label, with "Typus" printed with red ink. On the other hand, Carlos Bruch used to copy the names of the species described by other authors, to his own labels (several of them with a red frame, some with a green frame or without frame). He also used to attach a small piece of paper with the name of the species, cut from a handwritten letter from the author. The specimens of the Bruch collection labeled as 'Cotypus' have been considered as syntypes or as paratypes, depending on the context.

All the specimens labeled as types, or deemed to be types, bear a small reversed label "MACN-En 0000", that refer to a code used for the data base of the Museum collection, managed after "Aurora" (Rodríguez 2007/2012). We thank Mariel Guala for kindly help in managing "Aurora".
The collections at the MACN kept separately from the main collection are identified as follows: CBC, Carlos Bruch coll., and HBC, Hermann Burmeister coll. The specimens of the last collection, mostly labeled "Colección antigua" (Ancient Collection), conform the basis of the insect collection of the Museum.

Other institutional repositories are identified as follows: AMNH, American Museum of Natural History, New York, USA; BMNH, The Natural History Museum, London, UK; CMNC, Canadian Museum of Nature Collection; FMNH, Field Museum of Natural History, Chicago, USA; FUEDEI, Fundación para el Estudio de Especies Invasivas, (Hurlingham, Buenos Aires) (former SABCL) South American Biological Control Laboratory; IBSP, Instituto Biológico, São Paulo, Brasil; IFML, Instituto Fundación Miguel Lillo, Tucumán; INTA, Instituto Nacional de Tecnología Agropecuaria, Castelar, Argentina; IRSNB, Institut Royal des Sciences Naturelles de Belgique, Brussels, Belgium; MANC, Ministerio de Agricultura de la Nación, Departamento de Zoología Agrícola, at INTA; MCZC, Museum of Comparative Zoology of Harvard University, USA; MLP, Museo de La Plata, La Plata; MNNC, Museo Nacional de Ciencias Naturales, Santiago, Chile; MNHN, Muséum National d'Histoire Naturelle, Paris, France; MNRJ, Museu Nacional, Rio de Janeiro, Brazil; MZSP, Museu de Zoologia da Universidade de São Paulo, Brasil; NZAC, New Zealand Arthropod Collection, Auckland, New Zealand; SMTD, Staatliches Museum für Tierkunde, Dresden, Germany; USNM, United States National Museum, Washington D.C., USA; ZMHB, Zoologisches Museum für Naturkunde der Humboldt Universität zu Berlin, Germany.

We have also mentioned some private collections, most of them currently kept at different institutions: ABC, Adolfo Breyer coll., at MACN; AMC, Antonio Martínez coll., chiefly at CMNC; ARR, Ennio Arrigutti coll., at USNM; BON, Gregorio Bondar coll., at AMNH; CPC, Carlos Porter coll., at MNCN; CSC, Campos Seabra coll., at MNRJ; CWOB, Charles O'Brien coll. Arizona, USA; GKC, Guillermo Kuschel coll., at NZAC; HAHC, Anne and Henry Howden coll., Ottawa, Canada; JB, Jean Brèthes coll.; JHRC, José Haedo Rossi coll., at IFML; HUS, Alphonse Hustache coll., at MNHN; JBC, Juan Bosq coll. at MLP; LMC, Eugène Le Moult coll., at ZMHB; MHC, M. Hagedorn coll., at BMNH; MOS, Julius Moser coll., at ZMHB; MVC, Manuel Viana coll., in part at MLP, other part in J.E. Barriga coll. Chile 
Abbreviations used: allot., allotypus, allotype; coll., collection; cot., cotypus-pi, cotype-s; f., figure-s; handwr., handwritten; holot., holotypus, holotype; lectot., lectotypus, lectotype; leg., legit, legint; p., page-s; paralect., paralectotypus-pi, paralectotype-s; parat., paratypus-pi, paratype-s; pl., plate-s; print., printed; spec., specimen-s; synt., syntypus-pi, syntype-s, tab., tabula-ae, table-s.

\section{CATALOG OF THE TYPES}

\section{APIONIDAE}

argentinum [Apion] Béguin-Billecocq 1909: 464. Buenos Aires Prov., one spec.

Holot. on a card, "Rep. Argentina / Prov. Buenos Aires / 9.VII.1905 / C. Bruch" print., date handwr., black frame; "Typus" print. on pale green paper; "Apion / argentinum / L.B.B. tipo" handwr. by Bruch, red frame; "Col. / C. Bruch" print, black frame; "argentinu LBB" handwr., cut. "MACNEn / 10578” print., reverse. Ex CBC.

bolivianum [Apion] Brèthes 1910: 216. Bolivia. Three (all three?) synt. on cards, on separate pins, two of them "Bolivia" handwr. on green paper, reverse white; "Repreparo / M. Viana / I-1960" handwr.; a rectangular piece of red cardboard; "Apion / bolivianum / Brèthes" handwr. by Brèthes on whitish paper; we add "Syntypus" handwr. on red paper. The 3rd one idem but without locality label, we add "Syntypus?" handwr. on red paper. "MACN-En / 10504 [10505, 10506]” print., reverse. Ex JB.

breyeri (sub Breyeri) [Apion] Brèthes 1910: 215-216. Bolivia.

Three (all three?) synt. on cards on separate pins, two of them "Bolivia" handwr. on green paper, reverse white [on white paper]; "Repreparo / M. Viana / I-1960" handwr.; a rectangle of pink cardboard; "Apion / Breyeri / Brèthes" handwr. by Brèthes on whitish paper; "10352" [-]. We add "Syntypus" handwr. on red paper. The 3rd one without locality label, a small disk of pink paper; we add "Syntypus?" handwr. on red paper. "MACN-En / 10607 [10608, 10609]" print., reverse. Ex JB.

contrarium [Apion] Béguin-Billecocq 1909: 451. Buenos Aires Prov.

pauperculum [Apion] Béguin-Billecocq 1909: 462. Wibmer \& O'Brien 1986: $40=A$. contrarium fide Béguin-Billecocq 1911: 133.
One synt. on a card, "Rep. Argentina / Prov. Buenos Aires / 27.XI.1904 / C. Bruch" print., date handwr., black frame; "Typus" print. on pale green paper; "Apion / contrarium / L.B.B. tipo" handwr. by Bruch, red frame; "A. contrarium n. sp. LBB" handwr., cut. "MACN-En / 10586" print., reverse. Ex CBC.

desbordesi (sub Desbordesi) [Apion] BéguinBillecocq 1911: 131-132. Córdoba Prov.; two 우 from Bruch.

One synt. on a card, "Rep. Argentina / Prov. Cordoba / 190_ / C. Bruch" print., black frame; "Typus" print. on pale green paper; "Apion / Desbordesi / L.B.B." handwr. by Bruch, red frame; "A. Desbordesi" handwr. by BéguinBillecocq?, cut. "MACN-En / 10585" print., reverse. Ex CBC.

gandolfoi [Coelocephalapion] Kissinger 2005: 493. Argentina, La Rioja, near dique Los Sauces, holot. ${ }^{\top}$; 173 parat. Argentina, Formosa, Chaco, Santiago. del Estero, Catamarca, La Rioja, San Luis, La Pampa and Neuquen Provinces. Holot. in USNM, parat. one at FUEDEI.

Holot., two parat. on pointed cards, on separate pins, "Argentina / La Rioja / near dique / Los Sauces" print.; "GPS130 / 23.XI.2002 / Neser, Gandolfo / MacKay" print.; "emerged pods / Prosopis / flexuosa" print. One parat. on a pointed card, "Argentina / La Rioja / Rt *38 / nr. Chamical" print.; "GPS120 / Cordo, Gandolfo / Mac Kay" print.; "emerged pods / Prosopis / flexuosa" print. One parat. on a pointed card, "Argentina / Catamarca / Rt * $3820 \mathrm{~km} / \mathrm{N}$ Catamarca" print.; "GPS132 / 23.XI.2002 / Neser, Gandolfo / \& Mac Kay" print.; "emerged pods / Prosopis / nigra" print.; "Argentina, Catamarca $\mathrm{RN}^{*}$ / 38, $20 \mathrm{~km} \mathrm{~N}$. Catamarca / 23 nov. 2002 / Neser, Gandolfo \& McKay col. / Emerged from pods of / Prosopis nigra? / 132" print., double black frame, folded in four. All: "Holotype [Paratype] / Coelocephalapion / gandolfoi / Kissinger [print.] $\widehat{\delta}$ [ㅇ] [handwr.] on red paper. "MACN-En / 10610 (holot.) [10611 through 10614 (parat.)]" print., reverse.

Notes: In the original publication it is said that the holotype is at USNM and paratypes in FUEDEI. The holotype was deposited at MACN, not at USNM, according to current legislation in Argentina.

hilare [Apion] Béguin-Billecocq 1909: 450-451. Argentina.

One synt. on a card, "Rep. Argentina / Prov. 
Tucumán / IV.1897 / C. Bruch” print., date handwr., black frame; "Typus" print. on pale green paper; "Apion / hilare / L.B.B. tipo" handwr. by Bruch, red frame; "hilare n.s. L.B.B." handwr. by Béguin-Billecocq, cut. "MACN-En / 10583" print., reverse. $E x \mathbf{C B C}$.

importunum [Apion] Béguin-Billecocq 1909: 454-455. Buenos Aires Prov., two spec.

planiceps [Apion] Béguin-Billecocq 1911: 132. Nomen novum pro A. importunum BéguinBillecocq 1909 non Fall 1908.

One synt. on a card, "Rep. Argentina / Prov. Buenos Aires / 1.1899 / C. Bruch" print., date handwr., black frame; "Typus" print. on pale green paper; "Apion / planiceps / B. Bill. [handwr.] / C. Bruch determ. [print]" on white paper; "Apion / importunum / L.B.B. tipo" handwr. by Bruch, red frame; "importunum / n. sp. L.B.B." handwr. by Béguin-Billecocq?, cut. "MACN-En / 10580" print., reverse. Ex CBC.

incisicolle [Apion] Béguin-Billecocq 1909: 455456. Buenos Aires Prov., one spec.

Holot. on a card, "Rep. Argentina / Prov. Buenos Aires / III.1897 / C. Bruch" print., date handwr., black frame; "Typus" print. on pale green paper; "Col. / C. Bruch" print., black frame; "Apion / incisicolle / L.B.B. tipo" handwr. by Bruch, red frame; "incisicolle / n. sp. L.B.B." handwr. by Béguin-Billecocq?, cut. "MACN-En / 10572" print., reverse. Ex CBC.

ingratum [Apion] Béguin-Billecocq 1909: 461. Buenos Aires Prov., two spec.

One synt. on a card, "Rep. Argentina / Prov. Buenos Aires / 190_ / C. Bruch" print., black frame; "Typus" print. on pale green paper; "Col. / C. Bruch" print., black frame; "Apion / ingratum / L.B.B. tipo" handwr. by Bruch, red frame; "ingratum / n. sp. L.B.B." handwr. by BéguinBillecocq?, cut. "MACN-En / 10577" print., reverse. $E x$ CBC.

lativentre [Apion] Béguin-Billecocq 1909: 451452. Buenos Aires Prov.

One synt. on a card, "Rep. Argentina / Prov. Buenos Aires / III.1897 / C. Bruch" print., date handwr., black frame; "Typus" print. on pale green paper; "Apion / lativentre / L.B.B. tipo" handwr. by Bruch, red frame; "A. lativentre / n. sp. L.B.B." handwr. by Béguin-Billecocq?, cut. “MACN-En / 10575" print., reverse. Ex CBC.

mediocre [Apion] Béguin-Billecocq 1909: 463464. Buenos Aires Prov. salpingoides [Apion] Béguin-Billecocq 1911: 132. Nomen novum pro Apion mediocre BéguinBillecocq 1909 non Sharp 1889.

One synt. on a card, "Rep. Argentina / Prov. Buenos Aires / 2.II.1905 / C. Bruch" print., date handwr., black frame; "Typus" print. on pale green paper; "Apion / salpingoides / = mediocre L.B.B." handwr. by Bruch, red frame; "salpingoides B.B. = mediocre B.B." handwr. by BéguinBillecocq?, cut. "MACN-En / 10571" print., reverse. Ex CBC.

nigrocyaneum (sub nigro-cyaneum) [Apion] Béguin-Billecocq 1909: 456-457. Tucumán Prov. One synt. on a card, "Rep. Argentina / Prov. Tucuman / 20.I.1904 / C. Bruch" print., date handwr., black frame; "Typus" print. on pale green paper; "Apion / nigrocyaneum / L.B.B. tipo" handwr. by Bruch, red frame; "nigrocyaneum n. sp. L.B.B" handwr. by Béguin-Billecocq?, cut. "MACN-En / 10570" print., reverse. Ex CBC.

pauperculum [Apion] Béguin-Billecocq 1909: 462. Buenos Aires Prov., two spec.

contrarium [Apion] Béguin-Billecocq 1909: 451. Wibmer \& O'Brien 1986: $40=A$. pauperculum, fide Béguin-Billecocq 1911: 133.

One synt. on a card, "Rep. Argentina / Prov. Buenos Aires / 190_ / C. Bruch" print., black frame; "Typus" print. on pale green paper; "Apion / pauperculum / L.B.B." handwr. by Bruch, red frame; "pauperculum L.B.B" handwr. by Béguin-Billecocq?, cut. "MACN-En / 10587" print., reverse. $E x \mathbf{C B C}$.

rufonigrum (sub rufo-nigrum) [Apion] Béguin-Billecocq 1909: 460-461. Buenos Aires Prov.

Two synt. on cards, on separate pins; “ $O$ " [“ $\bigcirc$ ”] print.; "Rep. Argentina / Prov. Buenos Aires / 5.IV.1904 [190] / C. Bruch" print., date handwr., black frame; "Typus" print. on pale green paper; "Apion / rufonigrum / L.B.B. tipo" handwr. by Bruch, red frame; "rufonigrum / n. sp. L.B.B. +" handwr. by Béguin-Billecocq?, cut. "MACN-En / 10573 [10574]" print., reverse. Ex CBC.

simplex [Apion] Béguin-Billecocq 1909: 459. Buenos Aires Prov.

One synt. on a card, "Rep. Argentina / Prov. Buenos Aires / IV.1896 / C. Bruch" print., date handwr., black frame; "Typus" print. on pale green paper; "Apion / simplex / L.B.B. tipo" handwr. by Bruch, red frame; "simplex / n. sp. L.B.B." handwr. by Béguin-Billecocq?, cut. "MACN-En / 10576" print., reverse. Ex CBC. 
submaculatum [Apion] Béguin-Billecocq 1909: 456. Tucumán Prov.

Two synt. on cards, on one pin, "Rep. Argentina / Prov. Tucuman / 15.VII.1900 / C. Bruch" print., date handwr., black frame; "Typus" print. on pale green paper; "Apion/submaculatum/L.B.B. tipo" handwr. by Bruch, red frame; "A. submaculatum / n. sp. L.B.B" handwr. by Béguin-Billecocq?, cut. "MACN-En / 10581 [10582]" print., reverse. Ex CBC.

tucumanense [Apion] Béguin-Billecocq 1909: 458. Tucumán Prov., two spec.

One synt. on a card, "Rep. Argentina / Prov. Tucuman / 20.I.1904 / C. Bruch” print., date handwr., black frame; "Typus" print. on pale green paper; "Apion / tucumanense / L.B.B. tipo" handwr. by Bruch, red frame; "tucuman / n. sp. L.B.B" handwr. by Béguin-Billecocq?, cut. "MACN-En / 10579" print., reverse. Ex CBC.

ustum [Apion] Béguin-Billecocq 1909: 457-458. Tucumán Prov.

anisorhynchum [Apion] Gerstaecker 1854: Wagner 1912: $31=$ A. ustum.

One synt. on a card, "Rep. Argentina / Prov. Tucuman / XII.1909 / C. Bruch" print., date handwr., black frame; "Typus" print. on pale green paper; "Apion / ustum / L.B.B. tipo" handwr. by Bruch, red frame; "ustum / n. L.B.B" handwr. by Béguin-Billecocq?, cut. "MACN-En / 10584" print., reverse. Ex CBC.

\section{BRENTIDAE}

franciscoi [Monrosiaia] Haedo Rossi 1959: 101110, pl. I, II. Bolivia: Santa Cruz: Gutiérrez Prov: Nueva Moka, leg. Martínez III.1955, holot. ô, allot. + in HR, seven parat. in AMC.

Two parat. $\hat{\sigma}$, + on cards, on separate pins, "Bolivia / Dto. Santa Cruz / Pcia. Gutiérrez / Nueva Moka III.1955 / Leg. A. Martínez" handwr., "Paratypus" handwr. on red paper; "Monrosiaia / franciscoi / Haedo Rossi / Det. J.A. Haedo Rossi" handwr. on white paper. "MACNEn 10619 [10620]" print., reverse.

Note: According to Sforzi \& Bartolozzi (2004), holot. and allot. are in JHRC [now in IFML?], parat. are in AMC, and there is a 'cot.' at the AMNH.

similis [Stereodermus] Mantilleri \& Sforzi 2006: 19-21. Brasil: Nova Friburgo, holot. in MNHN, Argentina: Misiones Prov., seven parat. in several repositories, including MNHN.
Two parat. on pointed cards, on separate pins, abdomen and dissected parts ( $\hat{O}$ genitalia) glued to the same cards. One of them "Museum Paris / Misiones / Env. de San Ignacio / Villa Lutecia / E.R. Wagner 1910" print. on pale green paper, remainings of a black frame; "Avril" print. The other one "Museum Paris [print.] / Front. de l'Argentine / et du Bresil / Bords du Rio Iguazu / E.R. Wagner 1909 [handwr.]" on pale gray paper, remainings of a black frame; "Juillet" print. Both: "Stereodermus / similis n. sp. [handwr.] / A. Mantilleri det. 2005 [print,]" on white paper, remainings of a black frame; "PARATYPE" print. on red paper. "MACN-En / 10615 [10616]" print., reverse.

\section{ANTHRIBIDAE}

lateralis [Piezonomus] Jordan 1904: 276. Brasil: Goyaz: Jatahy, a series.

One synt. on a card, "Jatahy, / Prov. Goyas" print.; "Cotypus" handwr. by Bruch on pale green paper; "42890" handwr. (in register book same data); "Piezonomus / lateralis / Jord. 1904 [handwr. by Bruch] / C. Bruch determ. [print.]" on white paper; "Piezonomus / lateralis / Jord. 1904. Paratype" handwr. by Jordan? "MACN-En / 10665" print., reverse. Ex CBC.

magnus [Ptychoderes] Mermudes \& Napp 2004: 27. Brasil: Rondonia, Amapá, Amazonas, Manaus, Pará, Mato Grosso. Holot., 26 parat. at MNRJ.

One parat. pinned, "Paratipo" print. on red paper; "Bolivia / Rio Colorado / Hito Surutú [handwr. by Martínez] [obverse, black frame] / II-1964 / A. Martínez [handwr. by Viana] [reverse]"; "Ptychoderes magnus / Mermudes \& Napp" print. on white paper, black frame. "MACN-En / 10603" print., reverse.

nanus [Gymnognathus] Jordan 1904: 248-249. Brasil: Goyaz: Jatahy, a series.

One synt. on a card, "Jatahy / Prov., Goyas. Brésil" print. on green paper; "Cotypus" handwr. by Bruch on green paper; "Gymnognathus / nanus / Jord. 1904 [handwr. by Bruch] / C. Bruch determ. [print.]" on white paper; "Gymnognathus / nanus Jord. 1904. Paratype" handwr. by Jordan?; "42879" handwr. (in register book same data). "MACN-En / 10669" print., reverse. Ex CBC.

Note: The spec. is labeled "paratype" but it is here considered as a synt. because no type category was designated in the publication. 
obtusus [Nematrichus] Jordan 1904: 269-270, 271. Brasil: Goyaz: Jatahy, "a long series"; key. One parat. on a card, "Jatahy / Prov. Goyas" print.; "Cotypus" handwr. by Bruch on pale green paper; " 42893 " handwr. (in register book same data); "Nematrichus / obtusus / Jord. [handwr. by Bruch] / C. Bruch determ. [print.]" on white paper; "Nematrichus / obtusus Jord. 1904. Paratype" handwr. by Jordan? "MACN-En / 10666" print., reverse. Ex CBC.

peruanus [Cratoparis] Brèthes 1920: 44-45. Perú: Chanchamayo.

peruanus [Euparius]: Blackwelder 1947: 770.

Two synt. on cards, on separate pins, remounted, they have pinholes, "C.R. Vigil / Pérou" handwr. on whitish paper; "Repreparo / M. Viana / I.1960" handwr.; "Cratoparis / peruanus / Brèthes" handwr. by Brèthes on whitish paper. We add "Syntypus" print. on red paper. "MACNEn / 10617 [10618]” print., reverse. Ex JB.

scutellaris [Eusphyrus] Jordan 1904: 290-291. Brasil: Goyaz: Jatahy, a series.

[scutellaris] [Ormiscus]: Valentine 1960: 6263. = Eusphyrus.

One synt. on a card, "Jatahy / Prov. Goyas. Brésil / Déc. 97 - Janv. 98" print. on green paper; "Cotypus" handwr. by Bruch on pale green paper; " 42896 " handwr. (in register book same data); "Ormiscus / scutellaris / Jord. 1904 [handwr. by Bruch] / C. Bruch determ. [print.]" on white paper; "Ormiscus / scutellaris Jord. 1904. Paratype" handwr. by Jordan? "MACN-En / 10670" print., reverse. Ex CBC.

secundus [Brachytarsus] Wolfrum 1931: 75. Buenos Aires Prov., from Bruch $\partial^{\lambda},+$.

Two synt. on cards, on one pin, "Rep. Argentina / Prov. Buenos Aires / 190_/ C. Bruch" print., black frame; "Typus" handwr. by Bruch on pale green paper; "Brachytarsus / secundus / Wolfr." handwr. by Bruch, green frame; "Brachytarsus / secundus / Wolfr. n. spec." handwr. by Wolfrum?, folded. "MACN-En / 10594 [10595]" print., reverse. Ex CBC.

vestitus [Homalorhamphus] Haedo Rossi \& Viana 1956: 22-26, f. 1, 2. Bolivia: Santa Cruz Departm,: Sara Prov., leg. Steinbach 1918, holot. $\partial^{\lambda}$, one parat. $\delta$ in MACN n ${ }^{\circ} 53791$. platyrhinus [Systaltocerus] Labram \& Imhoff 1840, fide Mermudes 2002: 585-590, f. 1-60. = Homalorhamphus vestitus.

Holot., one parat. pinned, "Bolivia. Sta. Cruz
/ Prov. Sara XI:1918 / J. Steinbach leg." print., black frame; "53791" handwr.; "Holotypus ["Paratypus"] print. on red [yellowish orange] paper; the holot. also "Homalorhamphus / vestitus / Rossi-Viana [handwr.] / Museo Argentino de Ciencias Naturales [print.]" on red paper. To the parat. we add "Homalorhamphus / vestitus / Rossi-Viana [handwr.] / Museo Argentino de Ciencias Naturales [print.]" on yellowish orange paper. "MACN-En / 10621 (holot.) [10622 (parat.)]" print., reverse.

vulgaris [Ormiscus] Jordan 1904: 288. Brasil: Goyaz: Jatahy. "A long series".

One parat. on a card, "Jatahy / Prov. Goyas. Brésil / Sept. à Nov. 97" print. on green paper; "Cotypus" handwr. by Bruch on pale green paper; "42895" handwr. (in register book same data); "Ormiscus / vulgaris / Jord. 1904 [handwr. by Bruch] / C. Bruch determ. [print.]" on white paper; "Ormiscus / vulgaris Jord. Paratype. 1904" handwr. by Jordan? "MACN-En / 10667" print., reverse. Ex CBC.

zeae [Brachytarsus] Wolfrum 1931: 74. Buenos Aires Prov. from Bruch, on dry corn ears.

zeae [Trigonorhinus]: Rheinheimer 2004: 103.

Seven synt. on cards, on three pins $(2+2+3)$, "Rep. Argentina / Prov. Buenos Aires / 28.VIII:1910 [190_, 28.VIII.1910] / C. Bruch" print., dates handwr., black frames;

"Typus" handwr. by Bruch on pale green paper; the 1st pin also "Brachytarsus / zeae / Wolfr." handwr. by Bruch, green frame; "Brachytarsus zeae / Wolfr. n. sp." handrwr. by Wolfrum?, cut. To the 2nd \& 3rd pins we add "Brachytarsus / zeae / Wolfrum 1931 / Syntypi" handwr. on red paper. "MACN-En / 10596 [10597 through 10602]" print., reverse. Ex CBC.

\section{PLATYPODIDAE}

mattai (sub Mattai) [Platypus] Brèthes 1919a: 26-27. Brasil: Manaos, leg. A.A. da Matta.

parallelus [Platypus] (Fabricius 1801): Wood \& Bright 1992: 1164-1168. = P. mattai and $=$ further 22 specific names.

parallelus [Euplatypus]: Wood 1993: 275.

One synt. on a card, remounted, it has a pinhole, "Da Matta / Manaus / II.1919" handwr. on whitish paper; "Repreparo / M. Viana / I-1960" handwr.; "Platypus Mattai Brèthes" handwr. by Brèthes on whitish paper. We add "Syntypus" print. on red paper. "MACN-En / 10623" print., reverse. Ex JB. 
plicatus [Platypus] Brèthes 1909: 225-226, f. 1, 2. Buenos Aires, several ${ }^{\prime}$, $\circ$; Corrientes Prov., leg. J.M. Huergo; Misiones Prov.

sulcatus [Platypus] Chapuis 1865: Blackwelder 1947: $791=$ P. plicatus.

mutatus [Platypus]: Wood \& Bright 1992: 11581159. = P. plicatus.

mutatus [Megaplatypus]: Wood 1993: 275.

Three synt.? on cards, remounted, "831 [826, _]"; "Repreparo / M. Viana / I-1960" handwr.; "Platypus / plicatus Brèthes" handwr. by Brèthes on whitish paper. We add "Syntypus?" handwr. on red paper. "MACN-En / 10624 [10625, 10626]" print., reverse. Ex JB. Two slides "Platypus / plicatus $\delta^{\lambda}[0]$ / Brèthes" handwr. by Brèthes, "420 [421]" handwr. with pencil. In slide coll. under P42 and P43.

Note: The spec. lack locality and type labels, setting doubt on their type condition.

triquetrus [Platypus] Brèthes 1909: 226-227, f. 3. Chaco Prov., one $\hat{\delta}$, one + .

parallelus [Platypus] (Fabricius 1801): Wood \& Bright 1992: $1164-1168 .=$ P. triquetrus and $=$ other 22 specific names.

parallelus [Euplatypus]: Wood 1993: 275.

Two slides " $\mathrm{N}^{\circ} 7652$ / Platypus / triquetrus

/ Brèthes / 우 [0]]" handwr. by Brèthes; "424"

["426"] handwr. with pencil. In slide coll. P44, P45. Ex JB.

Note: Spec. not found.

\section{SCOLYTIDAE}

acuteclavatus [Chramesus] Hagedorn 1909: 742-743. Argentina, from Bruch, one spec.

acuteclavatus [Pseudochramesus]: Blackwelder 1947: 785.

Holot.? on a card, "Rep. Argentina / Prov. Tucuman / 6.III.1906 / C. Bruch" print., date handwr., black frame; "Typus" print. on pale green paper; "Chramesus / acuteclavatus / Hgd. tipo" handwr. by Bruch, red frame; "Chramesus / acuticlavatus Hag." handwr. by Hagedorn?, cut. "MACN-En / 10633" print., reverse. Ex CBC.

Note : According to Bright (1992: 262) the holot. comes from La Plata; it was deposited at the Museum of Hamburg, and is lost, so setting doubt on the type condition of the specimen.

araujiae [Xyleborus] Brèthes 1921: 5-7, f. 1. 2 . [Buenos Aires Prov.]: La Plata 6.VIII.1921, on dry capsules of "tasi", Araujia sericifera (Asclepiadaceae).

araujiae [Neodryocoetes]: Schedl 1939: 412, f.
1, photo of a spec. of the type series housed at MACN, = longicollis Schedl fide Bruch.

araujiae [Araptus]: Wood \& Bright 1992: 953.

Ten synt. on two cards $(5+5)$, on separate pins, "La Plata / 7.IX.1921 / J.B. [J. Brèthes]" handwr. by Brèthes; "Xyleborus / araujiae Brèthes", "Type!" ["Tipo"] handwr. on whitish paper. One of these pins also "Ejemplar = Foto! C. Bruch 3.VI.1939" handwr. on greenish gray paper; a rectangle of red paper; "Neodryocoetes / araujiae (Brèth.) [handwr.] / Bruch determ. [print.]" on white paper. We add to both pins "Syntypi" handwr. on red paper. "MACN-En / 10638 [10639 through 10647]" print., reverse. Ex JB.

asperatus [Acorthylus] Brèthes 1922: 305. Buenos Aires 11.V.1909.

One synt. on a pointed card, "Bs. Aires / 11.V.09 / J.B." handwr., black frame; "Acorthylus / asperatus Brèthes", "Type!" handwr. by Brèthes; a rectangle of red paper. We add "Syntypus" print. on red paper. "MACN-En / 10648" print., reverse. Ex JB.

bruchi (sub Bruchi) [Hexacolus] Hagedorn 1909: 743. Argentina, many spec. from Bruch.

bruchi [Scolytodes]: Wood \& Bright 1992: 389.

One synt. on a card, "Rep. Argentina / Prov. Buenos Aires / 6.I.1905 / C. Bruch" print., date handwr., black frame; "M. Hagedorn" print. or typed with violet ink; "Typus" print. on pale green paper; "Hexacolus / Bruchi / Hged. tipo" handwr. by Bruch, red frame; "Hexacolus / Bruchi Hag." handwr. by Hagedorn?, cut. "MACN-En / 10631" print., reverse. Ex CBC.

Note: Six synt. from Buenos Aires Prov. are at MLP (del Río et al. 2005). After Wood \& Bright (1992: 389) the types were at the Museum of Hamburg and are lost.

espinosai (sub Espinosai) [Pityophthorus] Brèthes 1925: 202. Chile: Santiago, leg. M.R. Espinosa B., on a species of Synantheraceae.

Two synt. on cards, one on the venter, the other on the dorsum, on one pin, "M.R. Espinosa / En Sinantèrea / Santiago (Chile)" handwr.; "Pityophthorus / Espinosai Brèthes" handwr.; "type!" handwr., a rectangle of red paper. We add "Syntypi" handwr. on red paper. "MACNEn / 10661 [10662]" print., reverse. Ex JB.

Note: Specimens not found, seemingly lost after registration.

globosus [Chramesus] Hagedorn 1909: 742. Argentina, from Bruch, one spec. 
Holot. on a card, "Rep. Argentina / Prov. Santa $\mathrm{Fe} / 190 \_$/ C. Bruch" print., black frame; "Chaco" handwr. by Bruch; "Typus" print. on pale green paper; "Chramesus / globosus / Hged. tipo" handwr. by Bruch, red frame; "Chramesu / globosus Ha. " handwr by Hagedorn?, cut. "MACN-En / 10634" print., reverse. Ex CBC.

Notes: Although only one specimen was mentioned, five 'syntypes' from Misiones Province are housed at MLP (del Río et al., 2005). According to Wood \& Bright (1992: 265) the holotype comes from La Plata, it was at the Museum of Hamburg, and is lost.

nanus [Hypothenemus] Hagedorn 1909: 744. Argentina, from Bruch, two spec.

crudiae [Hypothenemus] (Panzer 1791): Wood \& Bright 1992: 914. = nanus.

One synt. on a card, "Rep. Argentina / Prov. Buenos Aires / 17[?].XI.1904 / C. Bruch" print., date handwr., black frame; "Typus" print. on pale green paper; "Hypothenemus / nanus / Hged. tipo" handwr. by Bruch, red frame; "Hypothen / nanus H." handwr. by Hagedorn?, cut. "MACNEn / 10632" print., reverse. Ex CBC.

Notes: According to Wood \& Bright (1992: 914) a syntype female from Argentina: La Plata, was at MLP, but it was not found (del Río et al. 2005).

nigrosetosus [Xylechinus] Hagedorn 1909: 737, f. 31. Neuquen Prov., at the mountain chain foot, from Bruch, three spec. ex MHC.

Three synt.? on cards, on two pins (1+2), "Rep. Argentina / Gob. Neuquen / III.1898 / C. Bruch" print., date handwr., black frame; "Typus" print. on pale green paper. The 1st pin also "M. Haged." print.? typed? with violet ink; "Xylesinus / nigrosetosus / Hgdrn. tipo" handwr. by Bruch, red frame. To both pins we add "Xylechinus / nigrosetosus / Hagedorn 1909 / Syntypus? [Syntypi?]" handwr. on red paper. "MACN-En / 10635 [10636, 10637]" print., reverse. Ex CBC. Notes: The description sets some doubt about the type condition. Wood \& Bright (1992) say the types are lost.

porteri (sub Porteri) [Phloeotribus] Bruch 1914: 25-27, pl. II: f. A-E. Chile, near Concepción, on plum-tree; Santiago Prov.: El Monte, on peachtree; in CBC, CPC.

Two synt. on cards, on separate pins, "Rep. Chile / 190_ / C. Bruch" print. black frame; "Typus" ["Cotypus"] print. on pale green paper. One of them also "Phloeotribus / Porteri / Bruch" handwr. by Bruch, red frame. To the other one we add "Phloeotribus / Porteri / Bruch 1914 / Syntypus" handwr. on red paper. "MACN-En / 10629 [10630]” print., reverse. Ex CBC.

porteri (sub Porteri) [Sinophloeus] Brèthes 1923: 434-435, f. 37. Chile: Cautín, on Nothofagus obliqua (Fagaceae).

destructor [Sinophloeus] Eggers 1942: Wood \& Bright 1992: 105. = S. porteri.

One slide "Sinophloeus / Porteri / Brèthes. type! antenne. Cautín, Chile. Porter leg. XI-1921" handwr. by Brèthes; " $\mathrm{N}^{\circ} 24$ ", handwr., in slide coll. under S20. Ex JB.

Note: Spec. not found.

porteri (sub Porteri) [Xylechinus] Brèthes 1925: 202-203. Chile: Loncoche, leg. Porter II.1917.

Two synt. on cards, on one pin, "Loncoche / Chili II.1917 / C.E. Porter" handwr.; "Xylechinus / Porteri Brèthes", "type!", handwr. on whitish paper; a rectangle of red paper. We add "Syntypi" handwr. on red paper. "MACN-En / 10663 [10664]" print., reverse. Ex JB.

Note: Spec. not found after it was registered; seemingly lost.

scaliger [Loganius] Hagedorn 1910: 5-6, f. 39. Argentina, from Bruch.

flavicornis [Loganius] Chapuis 1869: Blackwelder 1947: 788. = scaliger Hagedorn.

flavicornis [Cnemonyx]: Wood \& Bright 1992: 315. = L. scaliger Hagedorn, = Cnemonyx vianai Schedl 1951.

One parat.? on a card, "Rep. Argentina / Gob. Misiones / 2.VIII.1904 / C. Bruch” print., date handwr., black frame; "Typus" print. on pale green paper; "Loganius / scaliger / Hged.tipo" handwr. by Bruch, red frame; "Loganius / scaliger" handwr. by Hagedorn?, cut. "MACN-En / 10628" print., reverse. Ex CBC.

Note: According to Wood \& Bright (1992: 315) the holot. male of $L$. scaliger, from Argentina, was at the Hamburg Museum and is lost.

spinosus [Chramesus] Brèthes 1921: 167. f. 3, 4. On Acacia cavenia (Leguminosae), abundant in dry twigs, where they bore galleries.

cristatus [Chramesus] Schedl 1963: Wood \& Bright 1992: 269. $=$ C. spinosus Brèthes.

Three synt. (one of them damaged) on one card, there are three further adhesive drops without spec.; "Chramesus / spinosus Br. / Bs. As. / 16-II1921[hardly legible] (Type!!)" handwr. on a large card with rounded corners; "Col. J. / Brèthes" print., black frame. We add "Syntypi" handwr. 
on red paper. Six synt. on one card, "Buenos Aires / 15.II.1921 / J.B." handwr.; "Chramesus / spinosus Brèthes" handwr. by Brèthes; "types!" handwr. by Brèthes; a square piece of red paper; "ejemplares Fotos! C. Bruch / 3.VI.1939" handwr. by Bruch on pale green paper. We add "Syntypi" handwr. on red paper. Two synt. on one card, there are two further adhesive drops without spec., "Bs. As / IX.1921 / J.B.", "Chramesus / spinosus Brèthes", "dans Acacia cavenia", "Type !" handwr. by Brèthes; a square of red paper. We add "Syntypi" handwr. on red paper. One synt. on a card, there is a further adhesive drop without spec.; "Buenos Aires / 15 [?, hardly legible]. II.1921 / J.B.", "Chramesus / spinosus Brèthes" handwr. by Brèthes; "Tipos" handwr. on pale green paper. We add "Syntypi" handwr. on red paper. "MACN-En / 10649 [10650 through 10660]" print., reverse. Two slides "Chramesus / spinosus Brèthes Type! ex [vit dans] Acacia cavenia . Bs. As. / XII.1920 [IX.1921] / J.B. [J.B. leg.] handwr. by Brèthes. In slide coll. under $\mathrm{CH} 3, \mathrm{CH} 4$. Ex JB.

Note: The types, from La Plata, are mentioned as "not located" by Wood \& Bright (1992: 269). Not recorded by Blackwelder (1947).

\section{CURCULIONIDAE}

acuminatus [Adioristus] Hustache 1926: 191192. Chubut Prov., two spec. from Bruch. acuminatus [Cylydrorhinus]: Kuschel 1955: 289.

conspersus [Cylydrorhinus] (Waterhouse 1841) fide Kuschel 1958a: 240 = acuminatus Hustache, = araucanus Germain 1911 nom. nud., = humilis Germain 1911 nom. nud.

conspersus [Cylydrorhinus]: Wibmer \& O'Brien 1986: 104. = humilis, = acuminatus, = araucanus.

One synt. pinned, "Rep. Argentina / Gob. Chubut / 190_ / C. Bruch" print., black frame; "Typus" print. on pale green paper; " 218 " handwr. on pink paper; "Adioristus / acuminatus / Hust." handwr. by Bruch, red frame; "Adioristus / acuminatus m. O" handwr. by Hustache?, cut. "MACN-En / 9565" print, reverse. Ex CBC.

adspersus [Discophorus] Brèthes 1910: 224 225. Misiones Prov.

adspersus [Discophorellus]: Hustache 1936a: 213.

adspersoides [Macromerus]: Wibmer \& O'Brien (1986: 230). Nom. nov. pro Discophorus adspersus Brèthes 1910 non Fabricius 1787.
One synt. on a card, remounted (it has a pinhole), "Misiones" handwr. on green paper, reverse white; "Repreparo / M. Viana / I.1960" handwr.; "10250" handwr., red frame; "Discophorus / adspersus / Brèthes" handwr. by Brèthes on whitish paper. We add "Syntypus" print on red paper. “MACN-En / 10375” print., reverse. Ex JB.

aeneotinctus [Linomadarus] Hustache 1940b: 281-282. Bolivia: Yungas: Nor-Yungas, Caranavi V.1931, leg. Denier. Tucumán, from Bruch.

discalis [Linomadarus] Casey 1922, fide Kuschel 1983: 39 = L. aeneotinctus.

One synt. on a card, "Yungas / Bolivia" print.; "Typus" handwr. by Bruch on pale green paper; "Linomadarus / aeneotinctus / Hust." handwr. by Bruch, without frame; "Linomadarus / aeneotinctus m." handwr. by Hustache?, cut. "MACN-En / 9600" print., reverse. Ex CBC.

Notes: There is another spec. on a card, labeled "Typus" from Misiones, not mentioned in the description; see at the third list below, sub MACNEn / 9599. Five synt. are at MLP (Morrone \& Loiacono, 1994: 23).

affine [Tyloderma] Wibmer 1989: 50-52, f. 55, 97, 126, 136. Panamá: Tocumen (type local.), holot. in CWOB; parat. in several repositories, including MACN, from Argentina: Buenos Aires, Chaco, Corrientes, Formosa Prov.; Bolivia; Brasil; Colombia; Mexico; Panamá; Paraguay; Uruguay; Venezuela.

Six parat. on cards, on two pins $(3+3)$; " 27008 ", handwr. (in register book: San Isidro, prov. Buenos Aires, leg. Gemignani y Daguerre); "Paratypes / Tyloderma / affine / Wibmer 1989" print. on yellow paper. We add "Argentina [print.] / S. Isidro [handwr.] / Bs. Aires [print.] [obverse] / leg. Gemignani y Daguerre [reverse]". " MACN-En / 10227 [10228 through 10232]" print., reverse.

Note: One parat.is at MLP (Lanteri, Suárez \& del Río 2003).

affinis [Hyperodes] Hustache 1926: 203, 211212, pl. III: 3. Buenos Aires, from Bosq, Lizer \& Bruch.

affinis [Listronotus]: O'Brien 1979: 267.

lineolaticollis [Listronotus] (Blanchard 1851), fide Wibmer \& O’Brien 1986: 119 = L. affinis.

Two synt. on cards, on separate pins, "Rep. Argentina / Prov. Buenos Aires / 190_/ C. Bruch" print., black frame; "Typus" print. on pale green paper; one of them also "Hyperodes / affinis / Hust." handwr. by Bruch, red frame; "Hyperodes / affinis Hust." handwr. by Hustache?, cut. The 
other one also "Foto" print on pale green paper. We add "Hyperodes / affinis / Hustache 1926 / Syntypus" handwr. on red paper. "MACN-En / 9445 [9446]” print., reverse. Ex CBC.

affinis [Listroderes] Hustache 1926: 197, pl. II: f. 4 . In a key.

One synt. pinned., "Rep. Argentina / Gob. Rio Negro / 190_ / C. Bruch" print., black frame; "Foto", "Typus" print on pale green paper. We add "Listroderes / affinis / Hustache 1928 / Syntypus" handwr. on red paper. One synt. pinned, "Rep. Argentina / Prov. [print.] Rio Negro [handwr.] / H. Richter [print.]" black frame; "3657" handwr.; "Cotypus" print. on pale green paper; "19284" handwr. (in register book same data); "Listroderes / affinis / Hust." handwr. by Bruch, green frame; "146" handwr. on pink paper; "Listroderes / affinis / Hust. [handwr.] / Kuschel det. [print.] 1954 [handwr.]. One synt. pinned, a card with the abdomen; a plastic microvial with dissected parts; "Rep. Argentina / Prov. [print.] Rio Negro [handwr.] / H. Richter [print.]" black frame; "Typus" print. on pale green paper; "Bariloche" handwr.; "Listroderes /affinis / Hust." handwr. by Bruch, red frame; "213" handwr. on pink paper; "stroderes /affinis / m." handwr. by Hustache?, cut; "Listroderes / affinis Hustache / Morrone det. 1993" print. on white paper. "MACN-En / 9704 [9705, 9706]" print, reverse. Ex CBC.

affinis [Neohydronomus pulchellus var.] Hustache 1926: 230. "Cette forme, un peu plus large, plus convexe que le type, d'un revêtement différente, est peut-être une espèce distincte". Buenos Aires Prov., from Bruch.

affinis [Neohydronomus]: Wibmer \& O'Brien 1986: 189. Specific rank, as already suggested by Kuschel.

affinis [Neohydronomus]: Wibmer \& O'Brien 1989a: 298. Revision; specific rank

One synt., on a card "Rep. Argentina / Prov. Buenos Aires / 190_ / C. Bruch” print., black frame; "Typus" print. on pale green paper; "Neohydronomus / pulchellus / v. affinis / Hust." handwr. by Bruch, red frame; "Neohydrono / affinis" handwr. by Hustache, cut; "309" handwr. on pink peper. "MACN-En / 12438" print., reverse. Ex CBC.

albocaudatus [Phymatophosus] Hustache 1926: 256, pl. IV: f. 4. Tucumán Prov. XII.1903; Buenos Aires Prov., from Bruch.

Three synt. (all three?) on cards, on three pins. The 1st one: "Rep. Argentina / Prov. Tucuman / 190 / C. Bruch" print., black frame; "Typus", "Foto" print. on pale green paper; "Phymatophosus / albocaudatus / Hust." handwr. by Bruch, red frame; "Phymatophosus / albus m. *" handwr. by Hustache?, cut. The 2nd one: "Rep. Argentina / Prov. Tucuman / 190_/ C. Bruch" print., black frame; "Typus" handw̄r. by Bruch on pale green paper; "19266" handwr. [in register book same data, and "dádiva del Dr. Bruch" [a gift from Dr. Bruch]; "Phymatophosus / albus / Hust." handwr. by Bruch, green frame. We add "Phymatophosus / albocaudatus / Hustache 1926 / Syntypus?" handwr. on red paper. The 3rd one: "Rep. Argentina / Prov. Buenos Aires /190_ / C. Bruch" print., black frame; "Typus" print. on pale green paper. We add "Phymatophosus / albocaudatus / Hustache 1926 / Syntypus" handwr. on red paper. "MACN-En / 10102 [10103, 10104]" print., reverse. Ex CBC.

albus [Opseobaris] Bondar 1942b: 271-272.

alba [Opseobaris]: Hustache 1951b: 9. Corrientes Prov.: San Roque, from Bosq; [Santiago del Estero Prov.]: Rio Salado.

albus [Opseobaris] Bondar: Kuschel 1955: 274.= O. albus Hustache in litteris.

One synt. on a card, "Républ. Argentine / Chaco de Santiago / del Estero. Rio Salado" print.; "Typus" handwr. by Bruch on pale green paper; "Opseobaris / alba / Hust." handwr. by Bruch, without frame; "Opseobaris / alba m." handwr. by Hustache?, cut. Two synt.? on cards, on one pin, "Rep. Argentina / Prov. Corrientes / 190_/ C. Bruch" print., black frame; "Cotypus" print. on pale green paper; "Strongylotes / albus / Hustch." handwr. by Bruch, red frame. We add "Opseobaris / alba / Bondar1942 / Syntypus?" handwr. on red paper. "MACN-En / 10942 (typus) [10038 through 10041]" print. reverse. Ex CBC.

Note: There are two further spec. from Córdoba Prov., labeled "Cotypus", see the third list below.

americanus [Spartecerus] Hustache 1926: 171-172, pl. IV: 1. Santiago del Estero Prov.: Rio Salado, Wagner leg., type in HUS; Salta Prov. from Bruch.

americanus [Hustachius]: Marshall 1933: 63. Type species of the new genus.

Three synt.? parat.? on cards, on separate pins, "Rep. Argentina / Pr. Santiago d. Estero / 190 / C. Bruch" print., black frame; "Typus" ["Typus", "Cotypus"] print. on pale green pa- 
per. The 1st one also "Foto" print. on pale green paper. The 2nd one also "Spartecerus / americanus / Hust." handwr. by Bruch, red frame; "Spartecerus / americanus m." handwr. by Hustache?, cut; "100" handwr. on pink paper. The 3rd one "Rep. Argentina / Pr. Santiago d. Estero / 190_ / C. Bruch" print., black frame; "Cotypus" händwr. by Bruch on pale green paper; "19286" handwr.(in register book same data); "Spartecerus / americanus / Hust." handwr. by Bruch, green frame. To the 1st one we add "Spartecerus / americanus / Hustache 1926 / Syntypus" handwr. on red paper. "MACN-En / 10169 [10170, 10171]" print., reverse. Ex CBC. Note: The type condition is dubious, as no parat. were expressively mentioned in the original publication, and the holotype is said to be in HUS.

apicirostris [Conotrachelus] Brèthes 1910: 220-221. Bolivia.

One synt. on a card, remounted, "Bolivia" handwr. on green paper, reverse white; "Repreparo / M. Viana / I-1960" handwr.; "10243" handwr.; "Conotrachelus / apicirostris / Brèthes" handwr. by Brèthes on whitish paper. We add "Syntypus" print. on red paper. "MACN-En / 10354" print., reverse. $E x$ JB.

appendiculatus [Amathynetes] Kuschel 1949: 47-49, f. 6a-e, pl. III: f. 6. Chile: Arica: Parinacota, 4400 m; Cotacotani Lake 4550 m; Caquena 4400 m; Tacora: Aguas Calientes $400 \mathrm{~m}$. Bolivia:

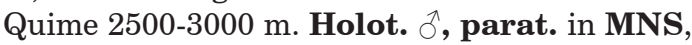
parat. in several repositories, including MACN. [appendiculatus] [Puranius]: Kuschel 1955: 288.

appendiculatus [Macrostyphlus]: Wibmer \& O’Brien 1986: 116.

appendiculatus [Amathynetoides]: Morrone 1994a: 30.

Two parat. on cards, on one pin, "Paratype" handwr. on yellow paper; "Arica / Parinacota 2900 m 29-2-48" handwr.; "52479" handwr.; "Amathynetes / appendiculatus / Kschl / Kuschel 1949" handwr. on white paper, black frame. "MACN-En / 10260 [10261]" print., reverse.

argentinensis [Baptobaris carnifex var.] Hustache 1939: 110. Widespread in Argentina. carnifex [Baptobaris] (Boheman 1836): Wibmer \& O'Brien 1986: 302.

One synt. on a card, "Rep. Argentina / Rio Salado / LeMoult. Paris XIII” print.; "Typus" handwr. by Bruch on pale green paper; "Baptobaris / carnifex / Boh. / var. argentinensis / Hust." handwr. by
Bruch, without frame; "Baptobaris carnifex Boh. v. / argentinensis m." handwr. by Hustache?, cut. "MACN-En / 9647" print., reverse. Ex CBC.

argentinensis [Ceutorrhynchus] Hustache 1926: 256-257. Buenos Aires Prov., from Bruch 10.X.1906.

argentinensis [Ceutorhynchus]: Wibmer \& O'Brien 1986: 276.

Three synt.? on cards, on separate pins, "Rep. Argentina / Prov. Buenos Aires / 190_ [2.1897, XI.1899] / C. Bruch" print., dates handwr., black frame; "Typus" print. on pale green paper, one of them also "Ceutorrhynchus / argentinensis / Hust." handwr. by Bruch, red frame; "284" handwr. on pink paper. To the other two we add "Ceutorrhynchus / argentinensis / Hustache 1926 / Syntypus" handwr. on red paper. "MACNEn / 9571 [9572, 9573]" print., reverse. Ex CBC. Note: The collectig dates differ, but otherwise the labels seem authentic.

argentinensis [Ericydeus] Lanteri 1995: 406407, f. 11, 38-44. Santiago del Estero Prov., holot. 7 , in USNM. Córdoba Prov., allot. $\odot$ in MLP. La Rioja Prov., parat. in MACN, MLP, BMNH.

One parat. pinned, "Dep. La Paz Rioja / M. Gómez" handwr. by Bruch; "Ericydeus / 16punctatus / (L.) [handwr.] / Kuschel det. 1957 [print.]". One parat. "Rep. Argentina / Prov. La Rioja / 190_/ C. Bruch" print., black frame. Both: "Paratype / Ericydeus / argentinensis / Lanteri 1995" print. on bright red paper. "MACN-En / 10084 [10085]" print., reverse. Ex CBC.

argentinensis [Eurhinus] Hustache 1926: 258. Misiones Prov., from Bruch.

Two synt. pinned, "Rep. Argentina / Gob. Misiones / 190_ / C. Bruch" print., black frame; "Typus" ["Cotypus"] print. on pale green paper. The "typus" also "Eurhinus / argentinensis / Hust." handwr. by Bruch, red frame; "289" handwr. on pink paper. To the "cotypus" we add "Eurhinus / argentinensis / Hustache 1926 / Syntypus" handwr. on red paper. "MACN-En / 9591 [9592]" print., reverse. Ex CBC.

argentinensis [Hadromorus (sic, pro Hadromerus] Hustache 1926: 157, pl. V: f. 2. [Córdoba Prov.]: Sierras de Córdoba: Alta Gracia, La Granja, on Acacia cavena (sic, pro caven) (Leguminosae); Santiago del Estero Prov., ô, $q$ from Bruch. One spec. from Misiones Prov.: Alto Paraná, ex Wagner, differs in having the dorsal vestiture golden yellow. 
argentinensis [Hadromeropsis]: Günther \& Zumpt 1933: 102.

argentinensis [Hadromeropsis (Hadromerop sis)]: Howden 1982: 60-62, f. 7-10, 161, 162, 181, 182, map 5. Designates lectot. $q$ \& one $\widehat{c}$, two paralectot. (both on one pin) from Córdoba Prov. in HUS (MNHN); one $q$ from Santiago del Estero in MNHN, one $\delta^{\lambda}$, one ${ }^{+}, " 30 "$, in MACN.

Lectot. on a card, "Alta Gracia. La Granja / Sierras de Córdoba / C. Bruch leg." print.; "Foto", "Typus" print. on pale green paper; "Hadromerus / argentinensis / Hust." handwr. by Bruch, red frame; "Lectotype. Hadromerus / argentinensis / Hustache / des. A. Howden" print. and handwr. on red paper. Two paralect. pinned, "Rep. Argentina / Pr. Santiago d. Estero / 190 / C. Bruch" print., black frame; "Paralectotype / Hadromerus argentinensis / Hustache / des. A. Howden" handwr. on yellow paper. Two paralect. on cards, on one pin, "Alta Gracia. La Granja / Sierras de Córdoba / II.922. C. Bruch leg." print, date handwr.; "Typus" print. on pale green paper. We add "Hadromerus / argentinensis / Hustache 1926 / Syntypi ?" handwr. on red paper. "MACN-En / 10181 (lectot.) [10182 through 10185 (paralect.)]" print., reverse. Ex CBC.

argentinensis [Hyperodes] Hustache 1926: 203, 208-209. Buenos Aires Prov.: San Isidro, from Bosq.

argentinensis [Listronotus]: O'Brien 1979: 267. Five synt. on cards, on two pins $(3+2)$, "Rep. Argentina / Prov. Buenos Aires / 190_ / C. Bruch" print., black frame; "Cotypus" handwr. by Bruch on pale green paper; "Hyperodes / argentinensis / Hust." handwr. by Bruch, without frame [with a green frame], the pin with three also "19250" (in register book same data). "MACN-En / 9488 [9489 through 9492]” print., reverse. Ex CBC.

argentinensis [Hyphantus] Hustache 1926: 156, pl. I: f. 1. Misiones Prov.: Alto Paraná: San Ignacio, leg. Wagner; Misiones Prov., from Bruch; Buenos Aires, from Bruch.

Two synt. on cards, on separate pins, "Rep. Argentina / Prov. Buenos Aires / 190_ [13. IV.1905] / C. Bruch" print., date handwr., black frame; "Cotypus" print. on pale green paper; "Hyphantus / argentinensis / Hustch." handwr. by Bruch, red [green] frame. The 1st one also "Hyphantus / argentinensis $\mathrm{m}^{*}$ " handwr. by Hustache? cut. The 2nd one also "19243" handwr. (in register book same data). "MACN-En /
19913 [10014]" print., reverse. Ex CBC.

Note: There is another spec. labeled "Foto" and "Typus", from Santiago del Estero, not mentioned in the description, see at the third list below.

argentinensis [Listroderes] Hustache 1926: 198, 201. Buenos Aires, from Bruch, Lizer, and Bosq, "nombreux spécimens".

apicalis [Listroderes] Waterhouse 1841, fide Kuschel 1950a: $13=$ L. argentinensis.

One synt. on a card, "Rep. Argentina / Prov. Buenos Aires / 189_ / C. Bruch” print., black frame; "Typus" print. on pale green paper; "Listroderes / argentinensis / Hust. / handwr. by Bruch, red frame; "Listroderes apicalis / Waterhouse / Det. J.J. Morrone 1988" print. on white paper. "MACN-En / 9694" print., reverse. Ex CBC.

Note: Identified in 1988 as L. apicalis Waterhouse by Morrone.

argentinensis [Sibinia] Hustache 1926: 234 235. Buenos Aires, from Bruch.

argentinensis [Paragoges]: Kuschel 1955: 311. argentinensis [Sibinia]: Clark 1978: 313.

Two synt. on cards, on separate pins, "Rep. Argentina / Prov. Buenos Aires / 15.VII.1917 [10.1894] / C. Bruch" print., dates handwr., black frame; "Typus" ["Cotypus"] handwr. by Bruch on pale green paper; "Sibinia / argentinensis / Hust." handwr. by Bruch, red [green] frame, the "typus" also "Sibinia / argentinensis" handwr. by Hustache?, cut; "148" handwr. on pink paper. The "cotypus" also "19259" handwr. (in register book the same data). "MACN-En / 9511 [9512]" print. reverse. Ex CBC.

argentinica [Baris] Hustache 1951a: 70. Buenos Aires, from Bruch; Martín García Island., from Bosq.

Three synt. on cards, on two pins, "Rep. Argentina / Prov. Buenos Aires / 190_/ C. Bruch" print., black frame; "Typus" handwr. by Bruch on pale green paper; "Baris / argentinensis Hust." handwr. by Bruch, green frame.The pin with one also " 243 " handwr. on pink paper. "MACN-En / 10035 [10036, 10037]" print. reverse. $E x$ CBC.

Note: The specific names differ, argentinica against argentinensis, but otherwise the labels seem authentic.

argentinica [Tonesia] Hustache 1951b: 18. Misiones Prov. VIII.1904, from Bruch $\mathrm{n}^{\circ}$ 125; 
Santa Fe Prov.: Fives Lille, leg. Weiser, from Bruch $\mathrm{n}^{\circ} 225$. "= Lyterius argentinensis $m$. in litt."

Four synt. on cards, on three pins $(2+1+1)$. The 1st pin: "Rep. Argentina/ Gob. Misiones / 3.VIII.1904 / C. Bruch" print., date handwr., black frame; "Typus" handwr. by Bruch on pale green paper; "Lyterius / argentinensis / Hust." handwr. by Bruch, without frame; "Tonesia / argentinensis / Hust." handwr. by Bruch, green frame; "125" handwr. on pink paper. The 2nd pin: "Misiones / (Iguazú) / C. Bruch VIII.1904" handwr. by Bruch; "Fototypus" handwr. by Bruch on pale green paper. The 3rd pin: "Fives Lille / S. Fé. Weiser" print.;"Typus" handwr. by Bruch on pale green paper: "Lyterius / argentinensis / Hust. [handwr. by Bruch] / C. Bruch determ. [print.]", without frame;"Tonesia / argentinensis / Hust." handwr. by Bruch, green frame; " 225 " handwr. on pink paper, we add "Tonesia / argentinica / Hustache 1951 / Syntypus [Syntypi]" handwr. on red paper. "MACN-En / 9595 [9596, 9597, 9598]" print., reverse. $E x \mathbf{C B C}$.

Notes: The names differ, argentinica against argentinensis but the labels seem authentic. There are two further spec. on one pin, from Chaco Prov., labeled "Typus", not mentioned in the description (see the third list below).

argentinicus [Cholus] Heller 1906: 16-17. Chaco Prov., XII.1897 from Bruch, in SMTD sub 17993; = Richteri Faust in schedulam.

One synt. pinned, "Rep. Argentina / Gob. Chaco / 190_ / C. Bruch" print., black frame; "Typus" print. on pale green paper. We add "Cholus / argentinicus / Heller 1906 / Syntypus" handwr. on red paper. "MACN-En / 10161" print., reverse. Ex CBC.

Notes: The spec. bears also a label reading "Ptinus / hirtithorax Pic / var. / desiré" handwr., cut, obviously put by error. There is another spec. labeled "Typus" from Misiones Prov., not mentioned in the publication; see the third list, below, sub MACN-En / 10160. The type is said to be in SMTD.

argentinicus [Compsus] Heller 1921: 28-29, f. 8. Santiago del Estero Prov., leg. Bruch ô, + . argentinicus [Plococompsus]: Hustache 1938a: 110 .

[argentinicus] [Oxyderces]: O'Brien \& Wibmer 1982: 7.

argentinicus [Oxyderces]: Wibmer \& O'Brien 1986a: 80 .
One synt. pinned, "Rep. Argentina / Pr. Santiago d. Estero / 190 / C. Bruch" print., black frame; “○” print.; "Typus" print. on pale green paper; "Compsus / argentinicus / Heller" handwr. by Bruch, red frame; "Compsus / argentin" handwr. by Heller?; cut. "MACN-En / 10053" print., reverse. $E x \mathbf{C B C}$.

argentinicus [Hilipus, sic, pro Heilipus] Heller 1921: 33-34, f. 11. Córdoba Prov. 14.II.1901; Catamarca and Tucumán Prov., from Bruch. argentinicus [Heilipodus]: Kuschel 1955: 295. One synt. pinned, "Rep. Argentina / Prov. Catamarca / 7.IV.1907 / C. Bruch" print., date handwr., black frame; "Typus" print. on pale green paper. We add "Hilipus / argentinicus / Heller 1921 / Syntypus" handwr. on red paper. "MACN-En / 9691" print., reverse. Ex CBC.

Note: There are two further spec. labeled "Typus" from Mendoza Prov., not mentioned in the description; see the third list below.

argentinus [Tachygonus] Viana 1949: 72-75, 82, f. 2. Misiones Prov.: Concepción: Santa María leg. Viana 1945, holot. $\delta^{\gamma}$, allot. $\rho$ in MACN $n^{\circ}$ 49303, slides 3001-3006; one parat. in MVC (in MLP).

Holot. $\delta$, allot. $\odot$ on cards, on separate pins, “”" [“”] print.; "Misiones Argentina / Dep. Concep. Sta. Maria / 1945. M.J.Viana” print., date handwr., black frame; "49303" handwr.; "Holotypus ["Allotypus"] print. on red paper; "Tachygonus / argentinus / Viana / Holotipo 万. [Alotipo +] [handwr.] / Museo Argentino de Ciencias Naturales [print.]" on red paper. The holot. also "Dibujado" [depicted] handwr. with red ink. "MACN-En / 10404 (holot.) [10405 (allot.)]" print., reverse. Six slides "Tachygonus / argentinus / Viana / Det. M. Viana" handwr. Two of them also "Paratipo", other two "Paratipo ㅇ [handwr.] / Museo Argentino de Ciencias Naturales / Sección Entomología [print.]"; "Argentina . Misiones . Dep Concepción . Santa María . 1945. Leg. M. Viana . $\mathrm{N}^{\circ} 3001$ [through 3006]", "Antena $\odot$ Paratipo", "Alas . Paratipo Q", "Patas posteriores", "Cabeza . Paratipo + ", "Abdomen . Paratipo ${ }^{\circ}$ " "Varias partes ejem. $\mathrm{N}^{\circ}$ 52378". In slide coll. under T72 through T77.

Note: One parat. $\delta$ is at MLP (Morrone \& Loiacono 1994: 20).

aspericollis [Menetypus] Hustache 1926: 150. Misiones: Bompland, from Bruch.

aspericollis [Pandeleteius]: Emden \& Emden 1939: 257. 
porosus [Pandeleteius]: Howden 1976: = aspericollis. Designates lectot. and paralectot. of M. aspericollis Hustache.

One paralect. on a card, "Misiones / Bompland" print.; "Typus" print. on pale green paper; "Menetypus / aspericollis / Hust." handwr. by Bruch, red frame. "MACN-En / 10012" print, reverse. $E x \mathbf{C B C}$.

aureolus [Adioristus] Hustache 1926: 186, 190-191, pl. II: f. 8. Mendoza Prov., type from Bruch; Tucumán Prov. XII.1899, one spec. from Bruch.

aureolus [Cylydrorhinus]: Wibmer \& O'Brien 1986: 103.

One synt. on a card, "Rep. Argentina / Prov. Mendoza / 190 / C. Bruch" print., black frame; "Foto", "Typus" print. on pale green paper; "Col./ C. Bruch" print., black frame; we add "Adioristus / aureolus / Hustache 1926 / Syntypus" handwr. on red paper. One synt. on a card, "Rep. Argentina / Prov. Tucuman / 190_ / C. Bruch" print., black frame; "Typus" print. on pale green paper; "310" handwr. on pink paper; "Adioristus / aureolus / Hust." handwr. by Bruch, red frame; "Adioristus / aureolus" handwr. by Hustache?, cut; "Antenistes / denticollis / Faust $\odot$ ?" handwr. by Bruch, red frame. "MACN-En / 9447 [9448]" print., reverse. Ex CBC.

Note: The label reading 'Atenistes denticollis' was probably attached by mistake.

auripes [Pantomorus] Hustache 1947b: 114, 125-126. Chaco de Santiago del Estero: Rio Salado, leg. Wagner (types); [Buenos Aires Prov.]: Tandil, leg. Viana; [Córdoba Prov.]: Alta Gracia: La Granja, from Bruch $\mathrm{n}^{\circ} 24$.

auripes [Pantomorus]: Wibmer \& O'Brien 1986: 64. $=$ prasinus Hustache 1947 = var. auratus Hustache 1947.

auripes [Naupactus]: Morrone 1995: 95. Nov. combin.

Two synt. on cards, on separate pins, "Alta Gracia. La Granja / Sierras de Córdoba / C. Bruch leg." print., "Typus" print. on pale green paper, one of them also "Pantoplanes / aureipes / Hust." handwr. by Bruch, red frame; "Votre $\mathrm{N}^{\circ} 24$ = Pantoplanes / aureipes" handwr. by Hustache, cut. To both we add "Pantomorus / auripes / Hustache 1947 / Syntypus" handwr. on red paper. "MACN-En / 10068 [10069]" print., reverse. Ex CBC.

Note: The specific name differs slightly from the publication, auripes against aureipes (wrong spelling), but otherwise the labels look authentic. aymaranus [Scotoeborus] Kuschel 1949: 37-38, pl. II: f. 9. Chile: Arica: Putre 3550 m, holot., parat. in MNNC, parat. in several repositories, including MACN.

aymaranus [Cylydrorhinus]: Wibmer \& O'Brien 1986: 103.

Three parat. pinned, "Parátipo" handwr. on or-

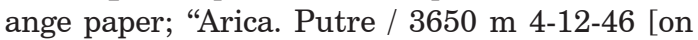
two, the other one without altitude]", handwr.; "42484" handwr., red frame; "Scotoeborus / aymaranus / Kschl" [on two, the other one also "Kuschel 1942", black frame] handwr. on white paper. "MACN-En / 10257 [10258, 10259]" print. reverse.

aysenensis [Cylydrorhinus melanoleucus subsp.] Kuschel 1958a: 248, f. 18. Chile: Aysén: Balmaceda. Argentina: Chubut Prov.; Neuquen Prov. Holot. ${ }^{1}$, allot. + , parat. in GK, parat. in MACN and MNNC.

melanoleucus [Cylydrorhinus]: Morrone 1995: 95. = subsp. aysenensis.

One parat. pinned, "10264" handwr.; "Neuquen" handwr. on green paper, reverse white, "64" handwr.; "Parátipo" print. on pale yellow paper; "Cylydrorhinus / melanoleucus / aysenensia n. ssp. / Kuschel det. 1957" handwr. and print. on white paper. One parat. pinned, " 4545 ", "65" handwr.; "Parátipo" print. on pale yellow paper; "Cylydrorhinus / melanoleucus / aysenensis n. ssp. [handwr.] / Kuschel det. 1957 [print.]" on white paper. "MACN-En / 10286 [10287]" print., reverse.

azurescens [Strangaliodes (Chersostrangalio des)] Kuschel 1949: 24-25, pl. II: f. 1. Chile: Arica: Tacora: Aguas Calientes $4400 \mathrm{~m}$; Humapalca $4300 \mathrm{~m}$; Alcérreca $4000 \mathrm{~m}$; Tahapaca 4900 $\mathrm{m}$; Caquena $4350 \mathrm{~m}$ (holot. $\hat{\delta}$, one parat. in MNNC); Parinacota $4400 \mathrm{~m}$; Cotacotani $4500 \mathrm{~m}$; Puquíos 3700 m. Perú: Huailillas 4300 m. Parat. in several repositories, including MACN, MLP. azurescens [Strangaliodes]: Wibmer \& O'Brien 1986: 87. = tarapacanus synon. nov.

One parat. on a pointed card, "Parátipo" handwr. on red paper: "Arica / Caquena / 4400 m 1-348" and "CHILE" handwr.; "49942" handwr.; "Strangaliodes / (Chersostr.) / azurescens / Kschl K48" handwr. on white paper, black frame. "MACN-En / 10253" print., reverse.

Note: Two parat. are at MLP (Morrone \& Loiacono 1994: 9).

baccharis [Pandeleteius] Kuschel 1949: 16-17, pl. I: f. 6. Chile: Tarapacá: Arica: Lluta Valley; 
Arica, Azapa Valley, Chaca Valley, Camarones Valley until Huancarane: Pisaguá: Tana Valley. Holot., parat. in MNNC, parat. in several repositories, including MACN, MLP.

Two parat. on pointed cards, on one pin, "Parátipos" handwr. on red paper; "Arica / Huancarane / 30-11-46" and "CHILE" handwr.; "49941" handwr.; "Pandeleteius / baccharis / Kschl., K48" handwr. on white paper, black frame. "MACN-En / 10251 [10252]" print. reverse.

Note: Two parat. are at MLP (Morrone \& Loiacono 1994: 4).

basalis [Brachygeraeus] Hustache 1950b: 5. Misiones Prov. from Bruch $\mathrm{n}^{\circ}$ 251. Paraguay: Hohenau.

basalis [Melampius]: Kuschel 1983: 40.

One synt. on a card, "Rep. Argentina / Gob. Misiones / 190 / C. Bruch" print., black frame; "Typus" print. on pale green paper; "251" handwr. on pink paper; "Brachygeraeus / basalis / Hust." handwr. by Bruch, without frame; "Brachygeraeus / basalis m." handwr. by Hustache? cut. "MACN-En / 9669" print., reverse. $E x$ CBC.

bellus [Conotrachelus] Hustache 1926: 251, pl. IV: f. 2. Buenos Aires Prov., from Lizer and Bruch.

brethesi [Conotrachelus] Hustache 1926: 254: Kuschel 1950b: 113. Nom. nov. pro lateralis Brèthes 1910 non Champion 1904; = C. bellus.

brethesi [Conotrachelus]: Wibmer \& O'Brien 1986: 156.

Two synt. on cards, on separate pins, "Rep. Argentina / Prov. Buenos Aires / 190_ / C. Bruch" print., black frame; "Typus" print. on pale green paper. One of them also "Foto" print. on pale green paper; "Conotrachelus / bellus / Hustch." handwr. by Bruch, red frame. To the other we add "Conotrachelus / bellus / Hustache 1926 / Syntypus" handwr. on red paper. "MACN-En / 10100 [10101]" print., reverse. Ex CBC.

bicoloripes [Tachygonus] Viana 1949: 75-77, 83, pl. I: f. 3. Corrientes Prov.: Santo Tomé leg. Pellerano, ex coll. Bruch, holot. $\hat{\delta}$. Misiones Prov.: Loreto, leg. Ogloblin, in MACN n ${ }^{\circ} 49304$ one parat. $\delta$. One parat. $\delta$ in MANC.

Holot., one parat. on cards, on separate pins, "Argentina / Prov. Corrientes / 190_ / C. Bruch" print., black frame; "S. Tomé / Coll. Bosq" handwr. by Bruch; "Col. / C. Bruch" print. with red ink, red frame; "49304” handwr.; "Prep. N³240" handwr.; "Holotypus" ["Paratypus"] print. on red [yellow] paper; "Tachygonus / bicoloripes / Viana / Holotipo ô [Paratipo ô] [handwr.] / Museo Argentino de Ciencias Naturales [print]" on red [yellow] paper, black frame. The holot. also "Dibujado" [depicted] handwr. with red ink. "MACN-En / 10407 (holot.) [10408 (parat.)]" print. reverse. One slide "Antena. Holotipo $\sigma^{\lambda}$ ejem. $n^{\circ}$ 49304. Argentina . Corrientes. Santo Tomé. Leg. G. Pellerano. N 3240”, “Tachygonus bicoloripes Viana. Det. M. Viana [handwr.] / Museo Arg. C. Naturales Sección Entomología [print.]". In slide coll. sub T 78.

binotatus [Derelomus] Bondar 1941b: 462-463, f. 20-22. 390 spec. Brasil: Espirito Santo: São Mateus: fazenda Babassú 12.VI.1941, on flowers of Attalea compta (Palmae), 'cotypi' in BON, MZSP, FMNH.

binotatus [Phytotribus]: Bondar 1949: 181.

binotatus [Derelomus]: Vaurie 1953: 8. Designates lectot. out from 140 'cot.'

binotatus [Tenires]: Wibmer \& O'Brien 1986: 198.

One paralect. on a pointed card, "mun. S. Mateus / faz. Babassu", "Estado do Espirito Santo / VI:12:41. Brazil / G. Bondar leg.", and "on flowers / Attalea compta" print.; "53521" handwr.; "Cotype / Derelomus / binotatus / Bond." print. on red paper. "MACN-En / 10250" print., reverse.

birabeni [Cylydrorhinus] Kuschel 1958a: 250251, f. 22. Rio Negro Prov.: Bariloche, Nahuel Huapi Lake; "Patagonia", holot. $\delta$, allot. + , one parat. in GK, two parat. in MACN, one parat. in MLP.

Two parat. pinned, "7926" handwr.; "Rio Negro" handwr. on green paper, reverse white; "Parátipo" print. on pale yellow paper; "Cylydrorhinus / birabeni / n. sp. / Kuschel det. 1957" handwr. and print. on white paper; " 5 " ["21"] handwr. "MACN-En / 10312 [10313]" print., reverse.

Note: One parat. is at MLP (Morrone \& Loiacono 1994: 9).

birabeni [Eurymetopus] Kuschel 1945a: 127128. Argentina: [Buenos Aires Prov.]: Punta Lara, holot. in MLP. La Plata one parat. in MLP. Buenos Aires Prov., two parat. in MACN.

One parat. on a card, "Olivos (B.A.) / C. Bruch leg." photographed from typed; "Eurymetopus / birabéni / Kschl. / Paratipo" handwr. on red paper. One parat. pinned, "Rep. Argentina / Prov. 
Buenos Aires / 190_ / C. Bruch" print., black frame; "Paratipo" handwr. on red paper. We add "Eurymetopus / birabeni / Kuschel 1945" handwr. on yellowish orange paper. "MACN-En / 10247 [10248]" print., reverse. Ex CBC.

Note: The holot. is at MLP (Morrone \& Loiacono 1994: 4).

boliviana [Achia] Clark, Burke \& McKay 2007:

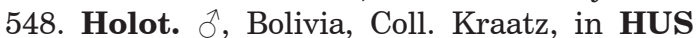
at MNHN; fourteen parat. from Bolivia and Argentina, Salta and Santiago del Estero Prov.

Eight parat. on pointed cards, on separate pins, "Argentina / Sgo. del Estero prov. / 30 Apr. 2006 [on five; 9 Jun. 2006 on three] / Cabrera col." print.; "Emerged from / seeds of / Urvillea chacoensis" print.; "Paratype / Achia / boliviana / Clark and Burke" print. on sky- blue paper, remainings of a black frame. One of them (of 9 June) also "Photo" handwr. "MACN-En / 6845 [6846 through 6852]" print., reverse.

bonariensis [Anchonoides] Brèthes 1910: 213214. Buenos Aires, several spec. in MACN.

ater [Hypselus] Boheman 1843: Hustache 1932: 137 = Anchonoides bonariensis.

Five synt. on cards, on two pins (2+3), "Buen. / Ayres" print. on green paper; "Anchonoides / bonariensis / Brèthes" handwr. by Brèthes on whitish paper. Pin with 2 also "Repreparo / M. Viana / XII-1959" handwr. We add "Syntypi?" handwr. on red paper. "MACN-En / 10295 [10296 through 10299]" print., reverse. Ex JB.

bosqi (sub Bosqi) [Baris] Hustache 1951a: 7475. [Buenos Aires Prov.]: Martín García Island., leg. Bosq, from Bruch n 356. Brasil: São Paulo.

Two synt. on cards, on separate pins, "I. Martín García / 1.X.1921. Bosq" ["Is. M. García / 1.X.921. Bosq"] handwr. by Bruch; "Typus" handwr. by Bruch on pale green paper. The 1st one also "Baris / Bosqi / Hust." handwr. by Bruch, without frame; "Baris Bosqi m." handwr. by Hustache?, cut. To the 2nd one we add "Baris / bosqi / Hustache 1951 / Syntypus" handwr. on red paper. "MACN-En / 9645 [9646]" print., reverse. $E x$ CBC.

bosqi [Cratosomus (Eucratosomus)] Kuschel 1945b: 361, 364-365, photo 1. Santiago del Estero Prov.: Forres III.1935 leg. Bosq, holot. ㅇ in MACN.

Holot. pinned, "S. del Estero / Forres. III-1935 [obverse] / Bosq [reverse]" handwr.; "Cratosomus / (Eucr.) bosqi / Kuschel / Holotipo" handwr. on red paper; "Cratosomus / (Eucratosomus) / bosqi / Kschl K 45 Tipo" handwr. on white paper with a graecan fret. "MACN-En / 10293" print., reverse.

bosqi (sub Bosqi) [Eugymnobaris] Hustache 1951a: 40-41. [Buenos Aires Prov.]: Martín García Island, from Bosq; Buenos Aires from Bruch 351.

One synt. on a pointed card, "Isla Martín García / 1.X.1921" handwr. by Bosq; "Typus" handwr. by Bruch on pale green paper; "Gymnobaris / Bosqi / Hust." handwr. by Bruch, without frame; "Gymnobaris / Bosqi m." handwr. by Hustache?, cut. One synt. on a card, "Rep. Argentina / Prov. Buenos Aires/190_/C. Bruch" print., black frame; "Isla Martín García / 1.X.921" handwr. by Bruch; "Typus" handwr. by Bruch on pale green paper; "Gymnobaris / Bosqi / Hust." handwr. by Bruch, green frame. To both we add "Eugymnobaris / Bosqi / Hustache 1951 / Syntypus" handwr. on red paper. "MACN-En / 10028 [10029]" print., reverse. Ex $\mathbf{C B C}$.

bosqi [Lissorhoptrus] Kuschel 1943: 307-315, f. 1:1, pl.: 4 f. Entre Ríos Prov.: Gualeguay, estancia El Rincón, on rice, leg. Bosq XII.1940, I.1941; Santiago del Estero Prov.: Campo del Cielo leg. Bosq I.1934; Jujuy Prov. 1 f from Bruch; Santa Fe Prov.: "Piquete, 2 parcitos" [two couples] leg. Bridarolli I.1928, 1931. Holot. $\delta$, allot. $\circ$ in MACN, parat. in MLP, GKC, JBC.

Holot., allot. on cards, on separate pins, "Gualeguay / E.R. XII-940 / J.M. Bosq" handwr.; "Col. / C. Bruch" print., black frame; "Lissorhoptrus / bosqi / Kuschel" handwr. on white paper; "Holotipo" ["Alotipo"] handwr. on orange paper. One parat. on a card, "Rep. Argentina / Prov. Jujuy / 190 / C. Bruch" print., black frame; "Col. / C. Bruch" print., black frame; "Paratipo" print. on orange paper; "bosqi n. sp. [handwr.] / Kuschel det. 1943 [print.]" on white paper; we add "Lissorhoptrus / bosqi / Kuschel 1943" handwr. on yellow paper. One parat. on a card, "Rep. Argentina / Pr. Santiago d. Estero / 190_ / C. Bruch" print., black frame; "Col. / C. Bruch" print., black frame; "Paratipo" print. on orange paper; "bosqi n. sp. [handwr.] / Kuschel det. 1943 [print.]" on white paper; we add "Lissorhoptrus / bosqi / Kuschel 1943" handwr. on yellow paper. "MACN-En / 10301 (holot.) [10302 (allot.), 10303, 10304 (both parat.)]" print., reverse.

Note: Thirty-one parat. are at MLP (Morrone \& Loiacono 1994: 17). 
bosqi (sub Bosqi) [Relistrodes] Hustache 1926: 216, pl. III: f. 5. In a key, Buenos Aires Prov. leg. Bosq; Santa Fe Prov. from Bruch.

[bosqi] [Anchodemus]: Kuschel 1950a: 14.

[bosqi] [Lixellus]: Burke 1963: 169.

bosqi [Lixellus]: Wibmer \& O’Brien 1986: 119. bosqi [Listronotus]: Morrone et al. 1995: 111.

One synt. pinned, "Rep. Argentina / Prov. Santa $\mathrm{Fe} / 190$ / C. Bruch" print., black frame; "Chaco" handwr. by Bruch; "Typus" print. on pale green paper; "Relistrodes / Bosqi / Hust." handwr. by Bruch, red frame; "Relistrod / Bosqi" handwr. by Hustache?, cut; "137" handwr. on pink paper. One synt. pinned, "Rep. Argentina / Prov. Buenos Aires / 190_/ C. Bruch" print., black frame; "Foto", "Typus" print. on pale green paper; we add "Relistrodes / Bosqi / Hustache 1926 / Syntypus" handwr. on red paper. "MACN-En / 9452 [9453]” print., reverse. Ex CBC.

bosqui (sub Bosqui, sic, pro bosqi) [Conotrachelus] Hustache 1924: 186. Buenos Aires Prov., several spec., type from Bosq in HUS. Bolivia: Trinidad X.1917, one spec.

bosqi (sub Bosqi) [Conotrachelus]: Hustache 1926: 186. In a key; Buenos Aires from Bosq \& Lizer, type in HUS; Buenos Aires Prov. from Bruch; Corrientes Prov.: San Roque from Bosq; Jujuy Prov. from Bruch; Bolivia: Trinidad.

bosqi [Pheloconus]: Wibmer \& O'Brien 1986: 171. C. Bosqui Hustache 1924 is a lapsus, corrected to Bosqi by Hustache (1926).

Six synt. on cards, on 3 pins $(1+2+3)$, "Rep. Argentina / Prov. Buenos Aires / 190_ / C. Bruch" print., black frame.The 1st \& 2nd pins: "Conotrachelus / Bosqi / Hustch." handwr. by Bruch. The 1st pin also "12" handwr. on pink paper; the 2nd pin also "Bosq" handwr.; "Cotypus" print. on pale green paper, to the 3rd pin we add "Conotrachelus / Bosqi / Hustache 1924 / Syntypus" handwr. on red paper. "MACNEn / 10113 through 10118 (all syntypes) ; 10111, 10112 (both syntypes?)" print., reverse. Ex CBC.

Notes: There are two further spec. labeled 'cotyp.', from Jujuy Prov., not mentioned in the descriptions (see the third list below). Five synt. are at MLP (Morrone \& Loiacono 1994: 15). The name honors Mr. Juan Bosq, consequently the epithet should read bosqi.

brachyderoides [Adioristus] Hustache 1926: 186, 188. Tucumán Prov.: Famaillá, leg. Weiser one spec.

brachyderoides [Scotoeborus]: Kuschel 1949: 31. brachyderoides [Cylydrorhinus]: Wibmer \& O’Brien 1986: 103.

Holot. pinned, "Famaillá / Tucumán / Weiser" handwr. by Bruch; "Typus" print. on pale green paper. We add "Adioristus / brachyderoides / Hustache 1926 / Holotypus" handwr. on red paper. "MACN-En / 9564" print., reverse. Ex CBC.

Note: There is another spec. labeled "Typus" from Tucumán, not mentioned in the publication; see the third list below.

brevipennis [Ovanius] Hustache 1950b: 97. Buenos Aires Prov., from Bruch n ${ }^{\circ} 248$.

Two synt. on a card [on a pointed card], on separate pins, "Rep. Argentina / Prov. Buenos Aires / VI.1897 / C. Bruch" print., date handwr., black frame; "Typus" handwr. by Bruch on pale green paper; "248" handwr. on pink paper; "Ovanius / brevipennis / Hust." handwr. by Bruch without frame [green frame].The 1st one also "Ovanius / brevipennis m." handwr. by Hustache?, cut. "MACN-En / 9534 [9535]” print., inverted. Ex CBC.

brevisquameum [Tyloderma] Wibmer 1989: 20, 73-74, f. 11, 66, 108, 133. Brasil: Mato Grosso, entrance to Transpantanal highway, $15 \mathrm{~km} \mathrm{~S}$ of Poconé (type locality) holot. in MZSP. Brasil; Venezuela, parat. in several repositories, including MACN.

Two parat. on pointed cards on separate pins, "Brazil / Mato Grosso. Entr. Transpantanal Hwy, 17 km S Poconé 24-4-1981 D.P. Wojeik" print.; "Paratype / Tyloderma / brevisquameum / Wibmer 1989" print. on yellow paper; "ex CWOB" handwr. "MACN-En / 10225 [10226]" print., reverse.

Note: Two paratypes are at MLP (Lanteri, Suárez \& del Río 2003).

breyeri (sub Breyeri) [Argentinorhynchus] Brèthes 1910: 211-212. Misiones Prov., ex ABC (in MACN).

nitens [Alhypera] Hustache 1926: 173.

breyeri [Cleonus (Argentinorhynchus)]: Csiki 1934: 67.

breyeri [Argentinorhynchus]: Kuschel 1950b: 112-113. = Alhypera nitens.

One synt. on a card, remounted, it has a pinhole, "Misiones" handwr. on green paper, reverse white; "Repreparo / M. Viana / I-1960" handwr., "10261" handwr.; "Syntypus" print. on red paper; "Argentinorhynchus / Breyeri Brèthes" handwr. by Brèthes on whitish paper blotted with ink. "MACN-En / 10377" print., reverse. Ex JB. 
breyeri (sub Breyeri) [Conotrachelus] Brèthes 1910: 218-219. Bolivia.

One synt. on a card, remounted, "Bolivia" handwr. on green paper, reverse white; "Repreparo / M.Viana / I-1960" handwr.; "10249" handwr.; "Conotrachelus / Breyeri Brèthes" handwr. by Brèthes. on whitish paper. One synt. on a card, remounted, "Bolivia. Travi R. 25.III.908" handwr. on whitish paper; "Repreparo / M.Viana / I-1960" handwr.; "Conotrachelus / Breyeri Brèthes" handwr. by Brèthes on whitish paper. To both we add "Syntypus" handwr. on red paper. "MACN-En / 10365 [10366]" print., reverse. Ex JB.

breyeri (sub Breyeri) [Cryptorhynchus] Brèthes 1910: 223. Bolivia.

breyeri [Zascelis]: Kuschel 1950b: 113

One synt. on a card, remounted, it has a pinhole, "Bolivia" handwr. on green paper, reverse white; "Repreparo / M.Viana / I-1960" handwr.; "10247" handwr.; "Cryptorhynchus / Breyeri Brèthes" handwr. by Brèthes on whitish paper. We add "Syntypus" print. on red paper. "MACNEn 10294" print., reverse. Ex JB.

\section{breyeri (sub Breyeri) [Heterobothroides]} Brèthes 1910: 225-226. Bolivia.

One synt. on a card, remounted, "Bolivia" handwr. on green paper, reverse white; "Repreparo / M.Viana / I-1960" handwr.; "10244" handwr.; "Heterobothroides / Breyeri Brèthes" handwr. by Brèthes on whitish paper. We add "Syntypus" print. on red paper. "MACN-En / 10376" print., reverse. Ex JB.

breyeri (sub Breyeri) [Mesocordylus] Brèthes 1910: 227. Misiones Prov.

cylindraceus [Mesocordylus] (Boheman 1845): Vaurie 1970: 29. $=$ M. breyeri.

coelomerus [Mesocordylus] (Chevrolat 1880): Vaurie 1970: 29. = M. breyeri.

One synt. on a card, remounted, it has a pinhole, "Misiones" handwr. on green paper, reverse white; "Repreparo / M.Viana / I-1960" handwr.; "10239" handwr.; "Typus" print. on red paper; "Mesocordylus / Breyeri Brèthes" handwr. by Brèthes on whitish paper, glued to a red label with "Museo Argentino de Ciencias Naturales" print.; a large piece of white cardboard "= type, $\lambda$, of Mesocordylus coelomerus Chevr. which may be synonym of $M$. cylindraceus Boh. P. Vaurie det. 1968". "MACN-En /10300" print., reverse. Ex JB. breyeri (sub Breyeri) [Rhyssomatus] Brèthes 1910: 221-222. Bolivia.

One synt. on a card, remounted, "Bolivia" handwr. on green paper, reverse white; "Repreparo / M.Viana / I-1960" handwr.; "10249" handwr.; "Rhyssomatus / Breyeri Brèthes" handwr. by Brèthes on whitish paper. We add "Syntypus" print. on red paper. "MACN-En / 10292" print., reverse. Ex JB.

breyeri (sub Breyeri) [Sternechus] Brèthes 1910: 212. Bolivia.

One synt. on a card, remounted, it has a pinhole; a glass microvial with dissected parts; "Bolivia" handwr. on green paper, reverse white; "Repreparo / M.Viana / I-1960" handwr.; "10252" handwr.; "Sternechus / Breyeri Brèthes" handwr. by Brèthes on whitish paper; "Syntypus" print. on pink paper. "MACN-En / 10371" print., reverse. $E x$ JB.

bruchi (sub Bruchi) [Adioristus] Hustache 1926: 186-187, pl. II: 7. Rio Negro Prov., leg. Richter, four spec., in CBC \& HUS.

bruchi [Acrostomus]: Kuschel 1955: 289.

One synt. pinned, "Rep. Argentina / Gob. Rio Negro / 190_ / C. Bruch" print., black frame; "Foto", "Typus" print. on pale green paper; "Adioristus / Bruchi / Hust." handwr. by Bruch, red frame. Two synt. pinned, "Rep. Argentina / Prov. Rio Negro / H. Richter" print. and handwr; one of them: "Bariloche" handwr.; "Cotypus" print. on pale green paper; we add "Adioristus / bruchi / Hustache 1926 / Syntypus" handwr. on red paper; the other one: "Typus" print. on pale green paper; "Adioristus / Bruchi / Hust." handwr. by Bruch, red frame; "dioristus / Bruchi m." handwr. by Hustache?, cut. "MACN-En / 9566 [9567, 9568]" print., reverse. Ex CBC.

bruchi (sub Bruchi) [Alhypera] Hustache 1926: 173, 175, pl. I: f. 7. Chaco de Santiago del Estero: Rio Dulce, January, types in HUS and LMC; Buenos Aires Prov. from Bruch; Santa Fe Prov: Fives Lille, leg. Weiser.

bruchi [Argentinorhynchus]: Kuschel 1950b: 112.

One synt. on a card, "Rep. Argentina / Prov. Buenos Aires / 190_ / C. Bruch" print., black frame; "Typus", "Foto" print. on pale green paper; "217" handwr. on pink paper; "Alhypera / Bruchi / Hust." handwr. by Bruch, red frame; "Alhypera / Bruch" handwr. by Hustache?, cut;. One synt. on a card, "Fives Lille / S. Fé. Weiser" print.; "Typus" print. on pale green pa- 
per. We add "Alhypera / bruchi / Hustache 1926 / Syntypus" handwr. on red paper. "MACN-En / 9513 [9514]” print., reverse. Ex CBC.

bruchi [Archopactus] Heller 1921: 23-24, f. 2. Tucumán Prov. III.1897; Santiago del Estero Prov. from Bruch.

bruchi [Naupactus]: Dalla Torre, Emden \& Emden 1936: 18.

Two synt. pinned, "Rep. Argentina / Prov. Tucuman / III.1897 [190_] / C. Bruch" print., date handwr., black frames; "Typus" print. on pale green paper; "Archopactus [Naupactus] / Bruchi / Heller [Hell. i.l. tip.]" handwr. by Bruch, red frame. One synt. pinned, "Trancas F.C.C.N. Tucum." handwr. by Bruch; "Cotypus" handwr. by Bruch on pale green paper; "Archopactus / Bruchi / Heller" handwr. by Bruch, green frame. We add "Syntypus?" handwr. on red paper. "MACN-En / 10061 (typus, 1897) [1062 (typus 190_), 10060 (cotypus)] print., reverse. Ex CBC. Note: The spec. labeled "Cotypus", from Tucumán: Trancas, was not explicitly mentioned in the description.

bruchi (sub Bruchi) [Brachygeraeus] Hustache 1950b: 4. Tucumán Prov. from Bruch n 261.

bruchi [Melampius]: Kuschel 1983: 40, 44.

Two synt. on cards, on separate pins, "Rep. Argentina / Prov. Tucuman / IV.1897 [III.1897] / C. Bruch" print., dates handwr., black frames; "Typus" handwr. by Bruch on pale green paper; "Brachygeraeus / Bruchi / Hust." handwr. by Bruch, without frame [with a green frame]; "256" handwr. on pink paper. One of them also "Brachygeraeus / Bruchi m." handwr. by Hustache?, cut. "MACN-En / 9685 (IV.1897) [9686 (III.1897)]" print., reverse. Ex CBC.

bruchi (sub Bruchi) [Coeliodes, sub Coeliodes] Hustache 1926: 257, pl. IV: f. 3. Tucumán Prov. 10.VII.1900 from Bruch.

bruchi (sub Bruchi) [Hypocoeliodes]: Hustache 1947a: 444.

Two synt.? on cards, on separate pins, head missing in one, "Rep. Argentina / Prov. Tucuman / V.1897 [190_] / C. Bruch" print., date handwr. black frames; "Typus" print. on pale green paper; "Coeliodes / Bruchi / Hust." handwr. by Bruch, red frame. One of them (without head) also "283" handwr. on pink paper, the other one also "Foto" print. on pale green paper. "MACN-En / 9569 [9570]" print., reverse. Ex $\mathbf{C B C}$.

Notes: The collectig date differs from that of the original description, setting doubt about the type condition of these spec. There is another spec. labeled "Typus" from Córdoba Prov.: Alta Gracia, not mentioned in the original description, see the third list below.

bruchi (sub Bruchi) [Coelonertus] Hustache 1950a: 47-48. Buenos Aires Prov.: Tandil, from Bruch $n^{\circ} 269$; Uruguay: Punta del Este, leg. Denier.

One synt. on a pointed card, "Tandil / Bs. As. Bruch" handwr. by Bruch; "Typus" handwr. by Bruch on pale green paper; "269" handwr. on pink paper; "Coelonertus / Bruchi / Hust." handwr. by Bruch, without frame; "Coelonertes / Bruchi m." handwr. by Hustache?, cut. "MACNEn / 9536" print., reverse. Ex CBC.

bruchi (sub Bruchi) [Eubulus] Hustache 1926: 236-237, pl. IV: f. 8. Buenos Aires Prov., from Bruch; San Isidro; Isla Santiago from Lizer y Trelles.

miniatus [Eubulus] (Boheman 1837): Kuschel 1950a: 18. = E. bruchi.

Eightsynt.oncards, onsixpins $(1+1+1+1+3+1)$, "Rep. Argentina / Prov. Buenos Aires / 25.V.1903 [190_, 20.II.1890, 10.XII.1900, 27.VI.1904, 25.V.1893] / C. Bruch" print., dates handwr., black frames; "Typus" ["Typus", "Cotypus", "Cotypus", "Typus", Cotypus"] handwr. on pale green paper. The 1st pin also "Eubulus / Bruchi / Hustch." handwr. by Bruch, green frame; "bulus / Bruchi" handwr. by Hustache?, cut, remainings of "m.". The 2nd pin also "Foto" print. on pale green paper. The 4th pin also "19280" handwr. (in register book same data); "Eubulus / Bruchi / Hust." handwr. by Bruch, green frame. The 5th pin also "19280" handwr. (in register book same data). To the 2nd, 3rd, 5th \& 6 th pins we add "Eubulus / Bruchi / Hustache 1926 / Syntypus [Syntypi]" handwr. on red paper. "MACN- En / 10143 [10144 through 10150]" print., reverse. Ex CBC.

bruchi (sub Bruchi) [Eugymnobaris] Hustache 1951a: 41. Santa Fe Prov.: Fives Lille, Bruch ${ }^{\circ}$ 224; [Buenos Aires Prov.?]: Salto.

One synt. on a card, "Fives Lille / S. Fé. Weiser" print.; "Typus" handwr. by Bruch on pale green paper; "Gymnobaris / Bruchi / Hust." handwr. by Bruch, green frame; "224" handwr. on pink paper. We add "Eugymnobaris / Bruchi / Hustache 1951 / Syntypus" handwr. on red paper. "MACNEn / 10026” print. reverse. Ex CBC. 
bruchi (sub Bruchi) [Listroderes] Hustache 1929: 197, 200, pl. II: f. 5. Catamarca Prov.: Hualfin, from Bruch \& Weiser, type deposited by Bruch in MOS.

One synt.? pinned, abdomen on a card, a plastic microvial with dissected parts, "Rep. Argentina / Prov. Catamarca / 5.IV.1907 / C. Bruch" print., date handwr., black frame; "Typus" print. on pale green paper; "112" handwr. on pink paper; "Listroderes / Bruchi / Hust." handwr. by Bruch, red frame; "istroder / Bruchi" handwr. by Hustache?, cut. "MACN-En / 9693" print., reverse. Ex CBC.

Note: The type is said to have been deposited in MOS, rending the type condition of the spec. doubtful.

bruchi (sub Bruchi) [Neochetina] Hustache 1926: 222-223, pl. III: f. 8. Buenos Aires Prov. from Bruch.

Two synt. on cards, "Rep. Argentina / Prov. Buenos Aires / 190_ / C. Bruch" print., black frame; "Typus" print. on pale green paper. One of them also "Foto" print. on pale green paper; "Neochetina / Bruchi" handwr. by Bruch, red frame. The other one also "Neochetina/ Bruchi" handwr. by Hustache?, cut; "144" handwr. on pink paper; "Neochetina / eichhorniae / Warner / Det. C.W. O'Brien 1977" handwr. and print. on white paper. "MACN-En / 9515 [9516]" print., reverse. Ex CBC.

Note: One of the synt. was identified in 1977 as Neochetina eichhorniae Warner 1970 by O'Brien.

bruchi (sub Bruchi) [Ochetina] Hustache 1926: 221, pl. III: f. 7. Buenos Aires Prov., a series from Bruch.

Five synt. on cards, on two pins $(1+4)$, "Rep. Argentina / Prov. Buenos Aires / 190_ [9.VII.1905] / C. Bruch" print., date handwr., black frame; "Typus" print. on pale green paper. The 1st pin also "Foto" print. on pale green paper, "Ochetina / Bruchi / Hust." handwr. by Bruch, red frame. The 2nd pin also "chetina / Bruchi" handwr. by Hustache?, cut; "141" handwr. on pink paper. We add "Ochetina / Bruchi / Hustache 1926 / Syntypi" handwr. on red paper. "MACN-En / 9517 [9518 through 9521]" print., reverse. Ex CBC.

bruchi (sub Bruchi) [Oxycorynus] Heller 1911: 7-8, f. 1a-d. Catamarca Prov. II.1900, from Bruch.

bruchi [Alloxycorynus]: Voss 1957: 101. Type species of the new genus.
Three synt. pinned, "Rep. Argentina / Prov. Catamarca / II.1910 / C. Bruch" print., date handwr., black frame; “ $O$ ” [“ㅇ", “○”] print; "Typus" ["Typus", "Cotypus"] print. on pale green paper. One of them also "Oxycorynus / Bruchi / Heller" handwr. by Bruch, red frame; "Alloxycorynus / Bruchi / (Heller) [handwr. by Kuschel] / det. G. Kuschel / 1992 [print.]" on white paper. To the other two we add "Oxycorynus / bruchi / Heller 1911 / Syntypus" handwr. on red paper. "MACNEn / 10564 [10588]" print., reverse. Ex CBC.

bruchi (sub Bruchi) [Paramadarus] Hustache 1926: 260, pl. V: f. 6. Entre Ríos Prov. 10.V.1911 from Bruch; Buenos Aires Prov.: Isla Santiago from Lizer y Trelles, \& Bruch, and from Bosq. complexus [Paramadarus] Casey 1922: Kuschel 1983: 41. = P. bruchi.

One synt. pinned, "Rep. Argentina / Prov. Buenos Aires / 190_ / C. Bruch" print., black frame, "Foto", "Typus" print. on pale green paper; "152" handwr. on pink paper; "Paramadarus / Bruchi / Hust." handwr. by Bruch, red frame; "darus / Bruchi n. s" handwr. by Hustache?, cut. One synt. pinned, "Rep. Argentina / Prov. Entre Ríos / 10.V.1911 / C. Bruch" print., date handwr. black frame; "Typus" print. on pale green paper; we add "Paramadarus / Bruchi / Hustache 1926 / Syntypus" handwr. on red paper. One synt. on a card, "Rep. Argentina / Prov. Buenos Aires / 3.1898 / C. Bruch" print, date handwr., black frame; "Typus" handwr. by Bruch on pale green paper; "19282" handwr. (in register book "III.1908"); "Paramadarus / Bruchi / Hust." handwr. by Bruch, green frame. "MACN-En / 9584 [9585, 9586]" print. reverse. Ex CBC.

bruchi (sub Bruchi) [Platyomus] Hustache 1926: 166, pl. I: f. 2. Chaco Prov. 1905, in CBC; Santiago del Estero Prov.: Rio Salado leg. Wagner, in HUS.

One synt.? pinned, "Sgo. del Estero / Rio Salado / Wagner col." print.; "Foto", "Typus" print. on pale green paper; "Platyomus / Bruchi / Hust." handwr. by Bruch, red frame. One synt.? pinned, "Rep. Argentina / Pr. Santiago d. Estero / 190_ / C. Bruch" print., black frame; "Typus" print. on pale green paper, we add "Platyomus / bruchi / Hustache 1926 / Syntypus" handwr. on red paper. "MACN-En / 9990 [9991]" print, reverse. Ex CBC.

Note: The type is said to be in HUS, rending the type condition of these spec. doubtful. 
bruchi (sub Bruchi) [Sphenophorus] Hustache 1936b: 112. Jujuy Prov. I.1920; Volcán, ex Weiser, and Bruch $\mathrm{n}^{\circ} 191$.

bruchi [Calendra]: Blackwelder 1947: 915.

rusticus [Sphenophorus] Gyllenhal 1838: Vaurie 1978: 17-19, f. 2, 4, 10, 21. = campestris (Chevrolat 1885), = nigroscutellatus (Chevrolat 1885), = bruchi.

Two synt. pinned, "Rep. Argentina / Prov. Jujuy / 1-15.I.1920 / C. Bruch" print., date handwr, black frame; "Volcan / Ing. Weiser [Weiser]" handwr. by Bruch; "Typus" print. on pale green paper. One of them also "Sphenophorus / Bruchi / Hust." handwr. by Bruch, red frame; "Sphenopho / Bruchi" handwr. by Hustache?, cut; "191" handwr. on pink paper. The other one also "Foto" print. on pale green paper; we add "Sphenophorus / Bruchi / Hustache 1936 / Syntypus" handwr. on red paper. "MACN-En / 10156 [10157]” print., reverse. Ex CBC.

bruchi [Sysciophthalmus] Heller 1906: 6-7, pl. I: f. 1, 2. Santa Cruz Prov., leg. Bruch II.1899. In SMTD sub 18397.

Two synt.? pinned, "Rep. Argentina / Gob. Santa Cruz / 190 / C. Bruch" print., black frame; "Typus" print. on pale green paper; "Sysciophthalmus / bruchi / Hell. ô [o+ tip." handwr. by Bruch, red frame. "MACN-En /10179 [10180]" print., reverse. Ex CBC.

Notes: The collectig dates differ, setting some doubt about the type condition; the spec. is labeled only by Bruch, not by Heller.

bruchiana [Ctenomyophila] Heller 1920: 240241, 1 f. Buenos Aires Prov.: Monte Veloz, leg. Bruch XI.1919, by thousands in burrows of the rodent Ctenomys talarum.

[bruchianus] [Hormops]: Kuschel 1964: 423.

bruchianus [Hormops]: Wibmer \& O'Brien 1986: 121.

Ten synt. on cards, on four pins $(1+3+3+3)$. The 1st pin: "Monte Veloz / Prov. Bs. Aires" handwr. by Bruch; "En nido de / Ctenomys talarum" handwr. by Bruch; "Foto", "Typus" print. on pale green paper; "Ctenomyophila / Bruchiana / Heller" handwr. by Bruch, red frame.The 2nd \& 3rd pins: "Rep. Argentina / Prov. Buenos Aires / 17.X.1919 / C. Bruch" print., date handwr., black frame; "ex nido de / Ctenomys" handwr. by Bruch; "Typus" and "Ctenomyophila / Bruchiana / Heller" handwr. by Bruch, red frame.The 4th pin: "Rep. Argentina / Prov. Buenos Aires / 30.XII.1912 / C. Bruch" print., date handwr., black frame; "Cotypus" handwr. by Bruch on pale green paper; "19248" handwr. (in register book same data); "Ctenomyophila / Bruchiana / Heller" handwr. by Bruch, green frame. "MACNEn / 9454 [9455 through 9463]" print., reverse. Ex CBC.

Note: Two synt. are at MLP (Morrone \& Loiacono 1994: 15).

brunnea [Costovia] Hustache 1950a: 31-32. Brasil: Jatahy.

One synt. on a card, "Jatahy / (Goyaz)" print. on pale gray paper; "Typus" handwr. by Bruch on pale green paper; "Costovia / brunnea / Hust." handwr. by Bruch, without frame; "Costovia / brunnea m." handwr. by Hustache?, cut. "MACNEn / 9537" print, reverse. Ex CBC.

brunneomaculatus [Enoplopactus] Hustache 1926: 158-159, pl. I: f. 3. Chaco de Santiago: Rio Salado, leg. Wagner, a series; Chaco Prov.: Tapenago (sic, pro Tapenagá?), a series.

Two synt. pinned, "Sgo. del Estero / Rio Salado / Wagner col." print.; "Foto", "Typus" ["Cotypus"] print. [handwr. by Bruch] on pale green paper; "Enoplopactus / brunneomaculatus / Hustch" handwr. by Bruch, red [green] frame; one of them also "19300" handwr. (in register book only name of species and "Cotipo"). One synt. pinned, "Rep. Argentina / Pr. Santiago d. Estero / 190 / C. Bruch" print., black frame; "Cotypus" print. on pale green paper, "Enoplopactus / brunneomaculatus / Hustch" handwr. by Bruch, red [green] frame; "Enoplopactus / brunneomaculatus" handwr., cut [seemingly "Hust."]. "MACNEn / 10050 [10051, 10052]" print., reverse. Ex CBC.

burmeisteri [Cylydrorhinus dentipennis subsp.] Kuschel 1958a: 245-246. = Otidores patagonicus Germain nomen nudum. Chile: Magallanes. Argentina: Santa Cruz Prov.: LagoViedma, Lago Argentino. Holot., allot. parat. in GK, parat. in several repositories, including MACN.

burmeisteri [Cylydrorhinus]: Wibmer \& O'Brien 1986: 103. Status nov.

Two parat. pinned, "Argentina / Santa Cruz. Lago [L.] Viedma / 23.2.48 Wittmer leg. [Wittmer]" handwr.; "Paratipo" print. on orange paper; "Cylydrorhinus / dentipennis / burmeisteri n. ssp. / Kuschel det. 1957" handwr. and print. on white paper. "MACN-En / 10282 [10283]" print., reverse.

Note: One parat. is at MLP (Morrone \& Loiacono 1994:10). 
campbelli [Pandeleteius] Howden 1976: 104-108, f. 156, 158, 167-175, 365, 367, 368, 409. Colombia: Magdalena; Santa Marta: San Lorenzo. Holot. $\hat{\text {, }}$, allot. + , many parat. in HOW and several other repositories

One parat. on a pointed card, "Colom. Magd. 7000 / San Lorenzo, $41 \mathrm{~km} \mathrm{~S} \mathrm{Sta.} \mathrm{Marta} \mathrm{/}$ V.2.1973 / Campbell \& Howden" photographed from typed; "Paratype / Pandeleteius / campbelli A.T. Howden" print. on yellow paper. "MACN-En / 10264" print., reverse.

carinirostris [Leptoschoinella $]$ Hustache 1951b: 44-45. Brasil: Jatahy.

schubarti [Angelocentris] Bondar 1950: Kuschel 1983: 35. = Leptoschoinella carinirostris.

One synt. on a card, "Jatahy / Brasil" handwr. by Bruch; "Typus" handwr. by Bruch on pale green paper; "Leptoschoinella / carinirostris / Hust." handwr. by Bruch, without frame; "Leptoschoinella / carinirostris m." handwr. by Hustache?, cut. "MACN-En / 9587" print., reverse. Ex CBC.

catamarcaensis [Baris] Hustache 1951a: 69. $=$ biseriata in litt.; from Bruch $\mathrm{n}^{\circ} 236$. No locality recorded.

Two synt. on cards, on one pin, "Hualfin / Catam. Weiser" handwr. by Bruch; "Typus" handwr. by Bruch on pale green paper; "Baris / biseriata / Hust." handwr. by Bruch, green frame; "236" handwr. on pink paper. We add "Baris / catamarcaensis / Hustache 1951 / Syntypi" handwr. on red paper. "MACN-En / 9623 [9624]" print., reverse. $E x$ CBC.

caudiculatus [Telurus] Morrone \& Anderson 1995: 8. Chile, Navarino Island, 31-XII-1962, holot. $\delta$ \& allot. $\$$ at MCZC; 22 parat. with same data as holot. 70 parat., Argentina, Tierra del Fuego \& Staaten Island; Chile: Navarino and Pilot Islands. These parat. in several repositories.

Two parat. in double mounting, with minutien and plastic sticks, "Argentina, T. del Fuego / 34: Ushuaia / Lapataia, 600 m / 2-3.ii.1979 / Misión Científica Danesa" print. on orange paper; "Telurus caudiculatus / Morrone \& Anderson / Paratype" print. on yellow paper."MACN-En / 10288 [10289]" print., reverse.

cavifrons [Brachystylus] Hustache 1926: 169171. Chaco de Santiago del Estero: Rio Salado, leg. Wagner, four spec.

cavifrons [Saurops]: Kuschel 1955: 309.
One synt. on a card, "Rep. Argentina / Pr. Santiago d. Estero / 190_ / C. Bruch" print., black frame; "Typus" print. on pale green paper; "Brachystylus / cavifrons / Hust." handwr. by Bruch, red frame; "rachystylu / cavifrons" handwr. by Hustache? cut. "MACN-En /10016" print., reverse. Ex CBC.

cayennensis [Ampeloglypter] Hustache 1951b: 63. French Guyana: Cayenne, from Aubert.

One synt. on a pointed card, "Cayenne / Col. Aubert" handwr. by Bruch; "Typus" handwr. by Bruch on pale green paper; "Ampeloglypter / cayennensis / Hust." handwr. by Bruch, without frame; "Ampeloglypter / cayennensis m." handwr. by Hustache? cut. "MACN-En / 9588" print., reverse. Ex CBC.

cervinus [Conotrachelus] Hustache 1926: 258, 255. Chaco de Santa Fe: Las Garzas, leg. Wagner; Chaco de Santiago: Rio Salado, leg. Wagner; Jujuy; Córdoba; Buenos Aires Prov., from Lizer \& Bruch.

cervinus [Conotrachelus]: Wibmer \& O'Brien 1986: 157. = pullus Fiedler 1940, fide Kuschel 1950.

Six synt. on cards, on four pins $(1+1+2+2)$. The 1st pin: "Rep. Argentina / Prov. Cordoba / 190_ / C. Bruch" print., black frame; the remainder three pins: "Rep. Argentina / Prov. Buenos Aires / 190_ [III.1897, 190_] / C. Bruch" print., date handwr., black frames. All four pins: "Cotypus" print. [print., print., handwr. by Bruch] on pale green paper. All pins except the 2nd one: "Conotrachelus [on the 3rd one: Conotrach.] / cervinus Hustch. [Hust.]" handwr. by Bruch, red frame [without frame, green frame]; 1st pin also: "onotrachelu / cervinus" handwr. by Hustache?, cut. To the 2nd pin we add "Conotrachelus / cervinus / Hustache 1926 / Syntypus" handwr. on red paper. "MACN-En / 10105 [10106 through 10110]" print., reverse. Ex CBC.

chilensis [Macrostylus (Amphideritus)] Kuschel 1949: 14-15, f. 1b, d, e, pl. I: f. 5. Chile: Tarapacá: Pisagua: Champaja $2900 \mathrm{~m}$; Iquique: Poroma $3000 \mathrm{~m}$. Holot. in MNNC, parat. in several repositories, including MACN.

chilensis [Amphideritus]: Wibmer \& O'Brien 1986: 55 .

One parat. pinned, "Parátipo" handwr. on red paper; "Pisagua. Champaja / 2900 m 162-48", "CHILE" handwr.; "49938" handwr.; "Macrostylus / (Amphideritus) / chilensis / Kschl. 
K48" handwr. on white paper, black frame. "MACN-En /10265" print., reverse.

Note: One $q$ parat. is at MLP (Morrone \& Loiacono 1994: 5).

cinereidorsum [Naupactus] Hustache 1947b: 92. Argentina; Uruguay.

One synt. pinned, "Stgo. Estero. 15.I.900" handwr.; "Typus" print. on pale green paper; "Naupactus / cinereidorsum / Hust." handwr. by Bruch, red frame; "Naupactus / cinereidorsum *" handwr. by Hustache?, cut. Two synt. pinned, "Rep. Argentina / Pr. Santiago d. Estero / 15.I.1900 / C. Bruch" print., date handwr., black frame; "Typus" print. on pale green paper; one of them also "Foto" print. on pale green paper. To both we add "Naupactus / cinereidorsum / Hustache" handwr. on red paper. "MACN-En / 9430 [9431, 9432]" print., inverted. Ex CBC.

Note: The paper suggests that there are further types, probably at the MNHN.

circumcinctus [Nicentrus] Hustache 1950b: 53. Santa Fe, Buenos Aires Prov., Canelones.

One synt. on a card "Bs. Aires / II.915" handwr. by Bruch; "Typus" handwr. by Bruch on pale green paper; "Madaropsis / circumcinctus / Hust." handwr. by Bruch, without frame; "Madaropsis / circumcinctus m." handwr. by Hustache?, cut; we add "Nicentrus / circumcinctus / Hustache 1950 / Syntypus" handwr. on red paper. One synt. on a card, "Rep. Argentina / Prov. Buenos Aires / 10.X.1906 / C. Bruch" print., date handwr., black frame; "Typus" handwr. by Bruch on pale green paper; " 266 " handwr. on pink paper; "Nicentrus / circumcinctus / Hust" handwr. by Bruch, green frame."MACN-En / 9680 [9681]" print. reverse. Ex CBC.

coarcticollis [Neobagous] Hustache 1926: 226, 227, pl. III: f. 9. Buenos Aires Prov., from Bruch.

Two synt. on cards, on separate pins, "Rep. Argentina / Prov. Buenos Aires / 190_ [16.X.1904] / C. Bruch" print., date handwr., black frames; "Typus" print. on pale green paper. One of them also "Foto" print. on pale green paper; "Neobagous / coarcticollis / Hust." handwr. by Bruch, red frame; the other one also "Neobagous / coarcticollis" handwr. by Hustache?, cut. "MACN-En / 9522 [9523]" print., reverse. Ex CBC.

coelestis [Linoma] Hustache 1951a: 12. Catamarca Prov. ex Bruch n 244.

One synt. on a card, "Rep. Argentina / Prov.
Catamarca / 190_/ C. Bruch" print., black frame; "Typus" handwr. by Bruch on pale green paper; "244" handwr. on pink paper; "Linoma / coelestis / Hust." handwr. by Bruch, without frame; "Linoma / coelestis m. desiré" handwr. by Hustache?, cut. "MACN-En / 9639" print., reverse. Ex CBC.

cordobaensis (sub cordobaense) [Baris] Hustache 1951a: 76-77. Córdoba Prov.: Alta Gracia, ex Bruch $n^{\circ} 235$.

One synt. on a pointed card, "Alta Gracia / Sierras de Córdoba / C. Bruch leg." print.; "Typus" handwr. by Bruch on pale green paper; "Baris / cordobaensis / Hust." handwr. by Bruch, without frame; "Baris / cordobaensis m." handwr. by Hustache?, cut. "MACN-En / 9644" print., reverse. Ex $\mathbf{C B C}$.

corumbanus [Nicentrus] Hustache 1950b: 5455. Brasil: Mato Grosso [do Sul]: Corumbá. chapadanus [Nicentrus] Casey 1922: Kuschel 1983: 40. = N. corumbanus.

Two synt. on cards, on separate pins, "Corumba / Matt. Grosso" print.; "Typus" handwr. by Bruch on pale green paper. One of them also "Nicentrus / corumbanus / Hust." handwr. by Bruch, without frame; "Nicentrus / corumbanus m." handwr. by Hustache?, cut. To the other one we add "Nicentrus / corumbanus / Hustache 1950 / Syntypus" handwr. on red paper. "MACNEn / 9682 [9683]” print., reverse. Ex CBC.

costalimai [Parallelosomus] Bondar 1943: 41-43. Two hundred spec. $\partial$,, , Brasil: surroundings of Bahia: Pitúba, V-VII.1942 on Rhynchospora scaberrima (Cyperaceae). 'Cotyp.' in BON and in several repositories.

costalimai [Parallelosomus]: Vaurie 1953: 14. Designates lectot. one from 75 cot. Other cot. in Brasil and Buenos Aires: Zool. Agríc., Minist. Agricult. (now INTA).

One paralect., pinned, "Pitúba, nos arredores Bahia” print.; "Estado da Bahia V-VII:'42. Brazil. G. Bondar leg." print., date handwr.; "on Rhynchospora scaberrima" print.; "53516" handwr.; "Cotype / Parallelosomus / costalimai / Bond." print. on red paper. "MACN-En / 10266" print., reverse.

costalimai [Polpones] Bondar 1943: 50. Sixtythree spec., $\hat{O}, \uparrow$ on flowers and leaves of

Rhynchospora cyperoides and $R$. scaberrima (Cyperaceae) in several districts of Bahia, Brasil. 'Cotyp.' in BON and in several repositories. 
costalimai [Polpones]: Vaurie 1953: 14. Designates lectot. one from 88 cot. (despite the original series is of only 63). Other cot. in São Paulo and Rio de Janeiro.

One paralect. on a pointed card, "Estado da Bahia, Brazil, G. Bondar leg.", "on Rhynchospora scaberrima or cyperoides" print.; "53519" handwr.; "Cotype / Polpones / costalimai / Bond." print. on red paper. "MACN-En / 10263" print. reverse.

costatum [Acanthobrachium] Brèthes 1910: 16. Bolivia.

costatus [Conotrachelus]: Kuschel 1950b: 113.

One synt. on a card, "Bolivia" handwr. on green paper, reverse white; "Acanthobrachium / costatum / Brèthes" handwr. by Brèthes on whitish paper; "10251" handwr. One synt. on a card, a small disk of red paper; "Acanthobrachium / costatum Brèth." handwr. on orange paper. Both: "Syntypus" print. on red paper; "Repreparo / M. Viana / I-1960" handwr. "MACN-En / 10369 [10370]" print., reverse. Ex JB.

crassirostris [Lichnus] Hustache 1940b: 297 (sic, pro 279). Córdoba Prov.: Alta Gracia, ex Bruch $n^{\circ}$ 293; Córdoba leg.Viana 1936; Chaco: Resistencia, leg. Denier 18.X.1936, "oscuro" (dark).

One synt. on a card, "Alta Gracia. La Granja / Sierras de Córdoba / C. Bruch leg." print.; "Typus" handwr. by Bruch on pale green paper; "29" handwr. on pink paper; "Lichnus / crassirostris / Hust." handwr. by Bruch, green frame. "MACN-En 10023" print., reverse. Ex CBC.

Note: One synt. is at MLP (Morrone \& Loiacono 1994: 24).

crinitus [Opseotapinotus] Heller 1921: 30-31, f. 9. Mendoza Prov. leg. Carette 10.V.1912.

One synt. on a card, "Rep. Argentina / Prov. Mendoza / 10.V.1912 / C. Bruch" print., date handwr., black frame; "Typus" print. on pale green paper; "Opseotapinotus / crinitus / Heller" handwr. by Bruch, red frame; "Opseotapinot / crinitus m." handwr. by Heller?, cut. "MACN-En / 10054" print., reverse. Ex CBC.

crucifer [Cryptorhynchus] Brèthes 1910: 224. Bolivia.

crucifer [Philonis]: Kuschel 1950a: 18.

One synt. on a card, remounted, "Bolivia" handwr. on green paper, reverse white; "Repreparo / M. Viana / I-1960" handwr.; "10245" handwr.; "Cryptorhynchus / crucifer / Brèthes" handwr. by Brèthes on whitish paper. We add "Syntypus" print. on red paper. "MACN-En / 10368" print., reverse. Ex JB.

cruralis [Acrostomus] Kuschel 1958a: 234-235, f. 5, 6. Chubut Prov.: Comodoro Rivadavia; Valdés Peninsula; other spec. without locality label. Holot. ${ }_{+}$, one parat. in MACN; allot. in USNM, one parat. in GKC.

Holot. pinned, "11630" handwr.; "Comodoro / Rivadavia" handwr. on green paper, reverse white; "Holotipo" print. on orange paper; "40" handwr. One parat. pinned, "Chu / but" print. on green paper; "41" handwr.; "Parátipo" print. on pale yellow paper. Both "Acrostomus / cruralis n. sp. [handwr.] / Kuschel det. 1957 [print.]" on orange paper. "MACN-En / 10380 (holot.) [10379 (parat.)]" print., reverse.

cryptonyx [Allomagdalis] Kuschel 1950c: 191192, f. 4, photo 8. Chile: Chillán; Bio-Bio: Laja Pond: Los Barros 1500 m. On Nothofagus pumilio (Fagaceae); Malleco: Pemehue $1300 \mathrm{~m}$; other spec. without locality label. Holot., parat. in MNNC, parat. in several repositories, including MACN.

Two parat. on pointed cards, on one pin, "Paratipo" handwr. on red paper; "L. Laja. L.Barros. 1500 m. 23.1.48" handwr.; "Chile" handwr.; "s/Nothofagus / pumilio" handwr.; "49936" handwr.; "Allomagdalis / cryptonyx / Kschl" handwr. on white paper, black frame. "MACN-En / 10267 [10268]" print., reverse.

cyphoides [Archopactus] Heller 1921: 24-25, f. 3. Tucumán Prov. XI.1899, from Bruch.

cyphoides [Naupactus]: Dalla Torre, Emden \& Emden 1936: 18.

cyphoides [Naupactus]: Wibmer \& O'Brien 1986: 59. = griseomaculatus Hustache 1923 fide Ward \& al. 1977; = prasinus Hustache 1947; = viridimarginalis Hustache 1947.

Two synt.? pinned, "Rep. Argentina / Prov. Tucuman / II.1906 [190 ] / C. Bruch" print., date handwr., black frame; "Typus" print. on pale green paper; one of them "Archopactus / cyphoides / Heller" handwr. by Bruch, red frame, to the other one we add "Archopactus / cyphoides / Heller 1921 / Syntypus" handwr. on red paper. "MACN-En / 10063 [10064]" print., reverse. Ex CBC.

Note: The collecting dates differ, setting some doubt about the type condition of the spec.

dahlbergiae [Cryptorrhynchus, sic, pro Cryptorhynchus] Hustache 1926: 237-238, pl. 
IV: f. 6. Misiones Prov., on Dahlbergia variabilis, whose grains they eat, from Bruch.

clitellarius [Discophorellus] (Boheman 1837): Kuschel 1955: 286. = Cryptorhynchus dahlgreniae, sic, pro dahlbergiae.

clitellarius [Troezon]: Wibmer \& O’Brien 1986: 229.

Four synt. on cards, on separate pins, "Rep. Argentina / Gob. Misiones / 1.1907 / C. Bruch" print., date handwr.; "Typus" print. on pale green paper. The 1st pin also "Cryptorhynchus / Dahlbergiae / Hustch." handwr. by Bruch, red frame; "yptorhynch / Dahlbergia" handwr. by Hustache?, cut. The 1st \& 2nd pins also "En semillas de (in seeds of) Dahlbergia variabilis" handwr. by Bruch. The 3rd pin also "Foto" print. on pale green paper. The 4th pin also "19275" handwr. (in register book same data); "Cryptorhynchus / dalbergiae / Hust." handwr. by Bruch, green frame. To the 2nd \& 3rd pins we add Cryptorrhynchus / dahlbergiae / Hustache 1926 / Syntypus" handwr. on red paper. "MACNEn / 9574 [9575, 9576, 9577]" print., reverse. Ex CBC.

dauci [Aulametopiellus] Brèthes 1926: 416. Buenos Aires, on carrots.

dauci [Hyperodes]: Hustache 1939: 48.

dauci [Listronotus]: O’Brien 1979: 267.

Three synt. on cards, remounted, on two pins $(1+2)$, "B. As / 15.I.926 [25.I.926]" handwr. by Brèthes; "Repreparo / M. Viana / I.1960" handwr.; "Aulametopiellus / dauci Brèthes" handwr. by Brèthes. We add "Syntypus" ["Syntypi"] handwr. on red paper. "MACN-En / 10372 [10373, 10374]" print., reverse. Ex JB.

decarloi [Tachygonus] Viana 1954: 158-162, f. 5, 6. Bolivia: Coroico: Huarinilla $1100 \mathrm{~m}$, leg. Kuschel 12.II.1949. Holot. ${ }^{\text {, one parat. }+9}$ in GKC. One parat ${ }^{\circ}$, slides 3044,3045 in MACN.

Four slides, in slide coll. under T79 through T82. T79: "Varias partes ejem. $\mathrm{N}^{\circ}$ Bolivia. Coroico. Huarimilla 1100 m. Leg. G. Kuschel. N $^{\circ}$ 3044" handwr.; "Tachygonus / decarloi / Viana Paratipo / Det. M. Viana X-1950" handwr. T80: "Cabeza. Paratipo. ejem. ${ }^{\circ}$ Bolivia. Coroico. Huarimilla 1100 m. Leg. G. Kuschel. N 3045. T81: "Genital ㅇ Holotipo. Ejem. $\mathrm{N}^{\circ}$ Bolivia. Coroico. Huarimilla 1100 m. Leg. G. Kuschel. $\mathrm{N}^{\circ} 3046$ ", "Tachygonus decarloi Viana. Genital ․ Holotipo. Det. M. Viana X-1950". T82: "Genital o. Paratipo. ejem. $\mathrm{N}^{\circ}$ Bolivia. Coroico. Huarimilla 1100 m. Leg. G. Kuschel. N 3047”,
“Genital . . Paratipo. Det. M. Viana X-1950". All handwr., with "Museo Arg. C. Naturales. Secc. Entomología" print. "MACN.En / 10565 [10566, 10567, 10568]" print., reverse.

deletangi (sub Deletangi) [Limnobaris] Hustache 1924: 104-105. Bolivia: Cuatro Ojos IX.1917, ô, ㅇ, types; Trinidad X.1917, one spec.

deletangi (sub Deletangi) [Sibariops]: Bondar 1943: 60, 64 .

deletangi [Oligolochus]: Kuschel 1983: 40.

One synt. on a pointed card, "Cuatro Ojos / Bolivia" print.; "Typus" handwr. by Bruch on pale green paper; "Sodesia / Deletangi / Hust." handwr. by Bruch, without frame; "Sodesia / Deletangi m." handwr. by Hustache?, cut. We add "Limnobaris / Deletangi / Hustache 1924 / Syntypus" handwr. on red paper."MACN-En / 9672" print., reverse. Ex CBC.

delicatulus [Centrinopus] Hustache 1950b: 121-122. Tucumán from Bruch $n^{\circ} 264$.

Four synt. on two double cards $(2+2)$, on one pin, "Rep. Argentina / Prov. Tucuman / 6.III.1900 / C. Bruch" print., date handwr., black frame; "Typus" handwr. by Bruch on pale green paper; "264" handwr. on pink paper; "Centrinopus / delicatulus / Hust." handwr. by Bruch, green frame."MACN-En / 9538 [9539, 9540, 9541]” print., reverse. Ex CBC.

densepunctatus [Heterosternus] Hustache 1951a: 28-29. Santa Fe Prov. from Bruch; Paysandú: La Noria, San Javier Stream leg. Bryant.

[densepunctatus] [Heterothorax]: Marshall 1946: 97. Nom. nov. pro Heterosternus Kirsch 1870 non Dupont 1832.

[densepunctatus] [Solenosternus]: Kuschel 1983: 42. Heterothorax Marshall $1946=$ Solenosternus Schoenherr 1826.

densepunctatus [Solenosternus]: Wibmer \& O'Brien 1986: 295.

One synt. on a card, "Estancia La Noria / Rio San Javier / Santa Fe, Argentina / G.E. Bryant. 10.1.1912" print., date handwr., a green horizontal line; "Typus" handwr. by Bruch on pale green paper; "Heterosternus / densepunctatus / Hust." handwr. by Bruch, without frame; "Heterosternus / densepunctatus m." handwr. by Hustache?, cut. "MACN-En / 9642" print., reverse. Ex CBC. 
dentatus [Adorenius, sic, pro Arodenius] Hustache 1926: 171, pl. IV: f. 5. Jujuy Prov.: La Quiaca, leg. Weiser, many spec.

dentatus [Arodenius]: Wibmer \& O’Brien 1986: 107. Adorenius: Hustache 1926 is a lapsus, fide Neave 1939: 64.

Four synt. on cards, on three pins $(2+1+1)$. The 1st \& 2nd pins: "Rep. Argentina / Prov. Jujuy / 25.III.1920 / C. Bruch" print., date handwr., black frame; "La Quiaca / Weiser" handwr. by Bruch; "Typus" handwr. by Bruch [print.] on pale green paper; "Adorenius / dentatus / Hust." handwr. by Bruch, green [red] frame; the 1st pin also "19246" handwr. (in register book same data); the 2nd pin also "Adoreniu / dentatus m" handwr. by Hustache? cut; the 3rd pin "La Quiaca / Jujuy. Weiser" handwr. by Bruch; "Foto", "Typus" print. on pale green paper. To all three pins we add "Adorenius / dentatus / Hustache 1926 / Syntypus [Syntypi]" handwr. on red paper. "MACN-En / 9695 [9696, 9697, 9698]" print., reverse. Ex CBC.

denticollis [Lissobaris] Hustache 1950b: 102. Perú: Zomora, from Ohaus; Marcapata, from Bang-Haas.

denticollis [Lissobaris]: Wibmer \& O'Brien 1986: 322. = castaneipennis Hustache 1950, fide Kuschel 1955: 274.

One synt., pinned, "ㅇ" handwr.; "Marcapata / Peru" print.; "Typus" handwr. by Bruch on pale green paper; "Lissobaris / denticollis / Hust." handwr. by Bruch, without frame; "Lissobaris / denticollis m." handwr. by Hustache?, cut. "MACN-En / 9542" print., reverse. Ex CBC.

denticulatus [Nertinus] Hustache 1951b: 7. Brasil: Goyaz: Jatahy, ex Donckier, a series.

Two synt. on pointed cards, on separate pins, "Jatahy / Brasil" handwr. by Bruch; "Typus" handwr. by Bruch on pale green paper; one of them: "Nertus / denticulatus / Hust." handwr. by Bruch, without frame; "Nertus / denticulatus m." handwr. by Hustache?, cut. To both we add "Nertinus / denticulatus / Hustache 1951 / Syntypus" handwr. on red paper. "MACN-En / 10030 [10031]" print., reverse. Ex CBC.

Note: Nertinus Marshall 1943 is a nomen novum pro Nertus Schoenherr 1844 non Bovie 1818.

dentipennis [Cylindrorrhinus] Burmeister 1879: 205. "Alto Rio Chico, cerca del pie de la cordillera" (High Rio Chico, near the foot of the mountain chain).

dentipennis [Cylydrorhinus]: Blackwelder 1947: 812 .
One synt. pinned, "Cylindror. / dentipennis / Bur. Tipo [handwr.] / Museo Argentino de Ciencias Naturales [print.]" on white paper; "Cylindrorh. / dentipennis / dentipennis / Burm. [handwr.] / Kuschel det. 1954 [print.]" on white paper. One synt. pinned "Elytrogonus / Vy. Bl. La." handwr. on white paper. We add "Cylindrorrhinus / dentipennis / Burmeister / Syntypus" handwr. on red paper. "MACN-En / 10210 [10211]" print., reverse. Ex HBC.

distinguenda [Liobaridia] Hustache 1951a: 10, 11. Catamarca Prov. from Bruch $n^{\circ} 253$, one spec.; Chaco de Santa Fe; Formosa Prov.: Nainec Lake; San Ignacio (in the key: distinguendas, sic, print error).

Two synt. on cards, on one pin, "Rep. Argentina / Prov. Catamarca / 1.1897 / C. Bruch” print., date handwr., black frame; "Typus" handwr. by Bruch on pale green paper; "Heterosternus / distinguendus / Hust." handwr. by Bruch, green frame; "252" handwr on pink paper. We add "Liobaridia / distinguenda / Hustache 1951 / Syntypi" handwr. on red paper. "MACN-En / 9640 [9641]" print., reverse. Ex CBC.

distortus [Hiotus (Melanolia)] Hustache 1950a: 39. Misiones Prov., ex Bruch $\mathrm{n}^{\circ} 285$. gentilis [Hiotus] Casey 1922: Kuschel 1983: 38. = H. distortus Hustache.

One synt. on a card, "Misiones" print.; "Typus" handwr. by Bruch on pale green paper; "285" handwr. on pink paper; "Hiotus / (Melanolia) / distortus / Hust. [handwr.] / C. Bruch determ. [print.]" on white paper, black frame. "MACNEn / 9543" print., reverse Ex CBC.

diversesquamulatus [Coelonertus] Hustache 1940b: 275-276. Misiones Prov. from Bruch; Buenos Aires Prov.: San Fernando leg. Denier 22.XII.1929; La Plata leg. Denier XII.1934.

diversesquamulatus [Coelonertus]: Wibmer \& O'Brien 1986: 289. = trifasciatus Hustache 1950, fide Kuschel 1955: 274.

One synt.? on a card, "Rep. Argentina / Prov. Buenos Aires / 3.1896 / C. Bruch" print., date handwr., black frame; "Typus" handwr. by Bruch on pale green paper; "121" handwr. on pink paper; "Coelonertus / diversesquamulatus / Hust." handwr. by Bruch, green frame. "MACN-En / 9544" print., reverse. Ex CBC.

Notes: The collecting dates differ, raising some doubt about the type condition. One synt. is at MLP (Morrone \& Loiacono 1994: 24). 
diversicollis [Rhyssomatus] Heller 1921: 34-35, f. 12. Santa Fe Prov.: Rosario, from Bruch.

One synt. pinned, "Rep. Argentina / Prov. Santa Fe / 190_ / C. Bruch" print., black frame; "Rosario" handwr. by Bruch; "Typus" print. on pale green paper; "O" print.; "Rhyssomatus / diversicollis / Heller" handwr. by Bruch, red frame; "Rhyssomatus / diversicollis m. . [handwr.] / Det. K.M. H. [print.]" on white paper, cut. "MACNEn / 10153” print., reverse. Ex CBC.

dorytomoides [Hyperodes] Hustache 1926: 203, 212, pl. III: f. 2. Buenos Aires Prov., from Bosq and from Bruch.

dorytomoides [Listronotus]: O'Brien 1979: 267.

Two synt. on cards, on separate pins, "Rep. Argentina / Prov. Buenos Aires / 30.I.1906 [20.I.1904] / C. Bruch" print., dates handwr., black frames; "Typus" print. on pale green paper. One of them also "Hyperodes / dorytomoides / Hust." handwr. by Bruch, red frame; "Hyperodes / dorytomoides" handwr. by Hustache?, cut; " 150 " handwr. on pink paper. The other one also "Foto" print. on pale green paper; we add "Hyperodes / dorytomoides / Hustache 1926 / Syntypus" handwr. on red paper. "MACN-En / 9465 [9466]" print., reverse. Ex CBC.

dubitabilis [Liobaridia] Hustache 1951a: 10, 11. Santa Fe Prov.; Misiones Prov, from Denier and Wagner. Paraguay: Hohenau.

One synt. on a card, "Santa Fe" handwr. by Bruch; "Typus" handwr. by Bruch on pale green paper; "Heterosternus / dubitabilis / Hust." handwr. by Bruch, without frame; "Heterosternus / dubitabilis m." handwr. by Hustache?, cut. We add "Liobaridia / dubitabilis / Hustache 1951 / Syntypus" handwr. on red paper. "MACN-En / 9643" print., reverse. Ex CBC.

dubius [Pantomorus (Graphognathus)] Buchanan 1942: 109. USA, Alabama, Mobile, holot. ㅇ, 21-VI-1940, USNM 56308. Parat. ㅇ Alabama: Mobile, Toulminville, Blakeley Island, Crichton and Prichard.

dubius [Graphognathus]: Buchanan 1947: 20. = leucoloma [Graphognathus] (Boheman 1840): Warner 1975: 856. = dubius Buchanan 1942; = imitator Buchanan 1947; = pilosus Buchanan 1942; = striatus Buchanan 1942.

leucoloma [Graphognathus]: Wibmer \& O'Brien 1986: 63. = dubius Buchanan 1942; = fecundus Buchanan 1947; = imitator Buchanan 1947; = pilosus Buchanan 1942; = striatus Buchanan
1942. Introduced in North America.

leucoloma [Naupactus]: Lanteri \& Marvaldi 1995: 219.

One parat. pinned, "Paratype / dubius. 56308 USNM" print. and handwr. on red paper; "Mobile, Ala. Blakely Id." handwr.; "TIT-9. Blakely Island. Mobile. 19" handwr. with pencil, figure in red, on a folded stripe of paper; "52490" handwr.; "Pantomorus / (Graphognathus) / dubius / Buch. / LB '42" handwr. "MACN-En / 10274" print., reverse

edax [Helodytes] Kuschel 1952a: 63-64. Misiones Prov., holot. + ex ABC in MACN 51368.

Holot. on a pointed card, "O" print.; "Argentina / Misiones", "Col. / A. Breyer" print.; "51368" handwr.; "Holotipo" print. on orange paper; "Helodytes / edax / Kuschel [handwr.] / Kuschel det. 1950 [print.]" on white paper. "MACN-En / 10290" print., reverse.

elaisae [Derelomus] Bondar 1941b: 461-462, f. 17-19. One hundred spec., $\hat{\sigma}, \uparrow$, reared from flowers of Elaeis guineensis (Palmae), Brasil: Bahia; 'cotypi' in BON and several other repositories, MACN not mentioned, name of Hustache 1940 in litteris.

elaeisae [Elaeidobius]: Kuschel 1952b: 272. Type species of the new genus.

elaisae [Elaeidobius]: Vaurie 1953: 16. Designates lectot. ex 22 'cot.'; other 'cot.' in FMNH, HUS and MZSP.

subvittatus [Elaeidobius] (Faust 1898): Kuschel 1955: 269, 310. Nova comb.; = E. elaeisae Bondar, African; = E. maynei Hustache 1924), African.

One paralect. on a pointed card, "Estado da Bahia / Brazil / G. Bondar leg." , "flores Elaeis guinensis" print; "53522" handwr.; "Cotypus / Derelomus / elaeisae / Hust." print. on red paper. "MACN-En / 10359" print., reverse.

Notes: Thirty-seven paralect. are at MLP (Morrone \& Loiacono 1994: 18). The specific epithet was spelled elaeisae by Kuschel.

elegantulus [Coelonertus (Crinacria) sic, pro Trinacria] Hustache 1950a: 49. Tucumán Prov. from Bruch $n^{\circ}$ 265; Misiones Prov.: Loreto, $n^{\circ}$ 121.

Two synt. on cards, on one pin, "Rep. Argentina / Prov. Tucuman / 1901 / C. Bruch" print., date handwr., black frame; "Typus" handwr. by Bruch on pale green paper; " 265 " handwr. on pink paper; "Trinacria / elegantula / Hust." handwr. by Bruch, green frame. "MACN-En / 9561 [9562]" print., reverse. Ex $\mathbf{C B C}$. 
elegantulus [Platyomus] Hustache 1923: 290291. Chaco de Santiago del Estero, type in HUS; Tucumán: Colmenar, in HUS. Bolivia: Lagunillas XI.1917, one spec.

One synt.? on a card, "Rep. Argentina / Prov. Tucuman / 1.900 / C. Bruch" print., date handwr., black frame; "Typus" print. on pale green paper; "Platyomus / elegantulus / Hust." handwr. by Bruch, red frame; "Platyomus / elegantulus" handwr., cut. "MACN-En / 10018" print., reverse. Ex CBC.

Note: The type from Tucumán is said to be in HUS, setting doubt about the type condition of the present spec. There is another spec. labeled "Cotypus" from Catamarca, not mentioned in the description, MACN-En / 10017, see the third list below.

elongatum [Tyloderma] Wibmer 1989: 20, 92-94, f. 17, 79, 121, 143. Uruguay: Colonia: San Pedro (type locality, holot. in CWOB). Argentina: Buenos Aires, Chaco, Corrientes, Entre Ríos, Formosa Prov. Bolivia: Santa Cruz. Brasil: Mato Grosso do Sul: Corumbá, Rio Grande do Sul. Paraguay. Uruguay, parat. in several repositories, including MACN.

Two parat. on pointed cards, "Uruguay. Colonia / San Pedro G.J. \& Z. Wibmer 6-I-79" print.; "treaded Ludwigia uruguayensis" print. One parat. on a card, "Entre Ríos / Concordia / Daguerre" print.; " 26253 " handwr. (in register book same data). Both: "Paratype / Tyloderma / elongatum / Wibmer 1989" print. on yellow paper. "MACN-En / 10233 [10234, 10235]" print., reverse.

Note: Three paratypes are at MLP (Lanteri, Suárez \& del Río 2003).

excelsus [Entimus] Viana 1968: 103-106, f. 1, 3. Brasil: Espirito Santo: Linhares: Parque Sooretama III.1959, XI.1967, leg. Campos Seabra, holot. ${ }^{\lambda}$, allot. $q$, two parat. + in MACN, other parat. in CSC, AMC.

Holot., allot., eight parat. pinned, one glued to a card "Brasil. E. Santo / Parque Sooretama / C. Seabra leg. [obverse] / III.1959. Linhares [reverse] [on holot. \& two parat.] / I-1967. Linhares [on allot. \& three parat.]”; “... A. Martínez leg. [obverse] XI-1967 - Linhares [reverse]" [on three parat.]. "Holotypus" ["Allotypus", "Paratypus"] print. on red [red, yellow] paper; "Entimus / excelsus / Viana / Holotipo ô [Alotipo + , Paratipo] handwr. on red [red, yellow] paper [obverse] / M. Viana X.1968 [reverse]". "MACN-En / 10451 (holot.) [10542 (allot.), 10543 through 10550 (all parat.)]" print., reverse. farinosus [Cylindrorrhinus] Burmeister 1879: 207. Once in Santa Cruz Prov.

farinosus [Cylydrorhinus]: Blackwelder 1947: 812.

Holot. pinned, "Cylindrorrhinus / farinosus / Bur. Tipus [handwr.] / Museo Argentino de Ciencias Naturales [print.]" on white paper, black frame. We add "Cylindrorrhinus / farinosus / Burmeister 1879 / Typus" handwr. on red paper; "Holotypus" print. on red paper. "MACNEn / 10198" print., reverse. Ex HBC.

farinosus [Opseotapinotus] Kuschel 1945a: 135-136. Catamarca: La Ciénaga, leg. Weiser, holot. $\odot$ in MACN.

Holot. pinned, "Cienaga / Catam. Weiser" handwr. by Bruch; "Opseotapinotus / farinosus / Kschl. Holotipo" handwr. on red paper. "MACNEn / 10378" print., reverse.

ferrugineus [Conotrachelus] Hustache 1926: 247, 254. Buenos Aires Prov., from Bruch and Bosq; Campana, from Lizer.

kirschi [Conotrachelus] Hustache 1936a: 28. Nom. nov. pro ferrugineus Hustache 1926 non Kirsch 1875. (Recorded also by Blackwelder 1947: 851)

cymba [Conotrachelus] Blackwelder 1947: 849. Nom. nov. pro ferrugineus Hustache 1926 non Kirsch 1875.

striatus [Conotrachelus] Hustache 1926: 247: Kuschel 1950a: 18. = ferruginea Hustache; = kirschi Hustache 1936.

striatus [Conotrachelus]: Wibmer \& O'Brien 1986: 169. = ferrugineus Hustache 1926 non Kirsch 1875, = kirschi Hustache 1936, = cymba Blackwelder 1947, Papp 1978.

One synt. on a card, "Rep. Argentina / Prov. Buenos Aires / 20.I.1904 / C. Bruch" print., date handwr., black frame; "Typus" print. on pale green paper; "Conotrachelus / ferrugineus / Hust." handwr. by Bruch, red frame; "notrachelus / ferrugineus m." handwr. by Hustache?, cut. "MACN-En / 10099" print., reverse. Ex CBC.

ferrugineus [Parallelosomus] Hustache 1940b: 297 [sic, pro 279]. Misiones Prov.: Paraná River. Brasil: Mendès; in a key; promises an extended description.

One synt. on a pointed card, "République Arg. ne / Rio Parana / Territoire des Missions" print., "Typus" handwr. by Bruch on pale green paper; "Parallelosomus / ferrugineus / Hust." handwr. by Bruch, without frame; "Parallelosomus / ferrugineus m." handwr. by Hustache?, cut. "MACN-En / 10024" print., reverse. Ex CBC. 
formosus [Entimus] Viana 1968: 2-6, 13, f. E, F, pl. I: f. 3. Misiones Prov.: Dept. Concepción: Santa María, leg. Viana X.1947, 53319; Corrientes Prov.: Santo Tomé, leg. Pellerano 53320; Candelaria, leg. Pellerano 53321. Holot. $\hat{\text {, }}$, allot. + , parat. two $\hat{\gamma}$, two $\circ$ in MACN; parat. two $\delta$, two $\circ$ in MVC, currently at MLP.

Holot., allot., two parat. pinned, "규" [“”, “ㄱ", "ㅇ"] print.; "Misiones Argentina / Dep. Concep. Sta María / X-1946. M.J. Viana" print., date handwr., black frame; "53319" handwr. Fourteen parat. pinned, one of them with $\delta$ genit. glued to a card; " $\widehat{O}$ [이" print.; "S. Tomé / Corrientes" print., diverse dates handwr.; " 53320 " handwr. One parat. pinned, " $\bigcirc$ " print.; "Misiones / Argentina / Puerto Bemberg / leg. A. Gallardo III.1951" handwr.; "53327" handwr. One parat. pinned, " 7 ", "Cande / laria 10.961" handwr.; "53321". All: "Holotypus", ["Allotypus", "Paratypus"] print. on red [red, yellow] paper; "Entimus / formosus / Viana / Holotipo ô [Alotipo , Paratipo]" handwr. on red [red, yellow] paper. "MACN-En / 10521 (holot.) [10522 (allot.), 10523 through 10540 (all parat.)]" print., reverse.

Note: Eight parat. $(4 \hat{\jmath}, 4$ P) are at MLP (Morrone \& Loiacono 1994: 10).

fortunai (sub Fortunai) [Cylindrorrhinus] Bruch 1940: 113-115, f. 7-9, pl. I: f. 1. Chubut Prov.: Cerro Negro 25.XII.1926, holot. in CBC (MACN).

fortunai [Cylydrorhinus]: Wibmer \& O'Brien 1986: 104.

Holot. pinned, "Cerro Negro / Chubut / 25.XII.1926 / J. Fortuna" handwr. by Bruch; "Fototipo" handwr, by Bruch on pale green paper; "Cylindrorrhinus / Fortunai / Bruch 1939 / C. Bruch determ." handwr. by Bruch, black frame. We add "Holotypus" print. on red paper. "MACN-En / 10158" print., reverse. Ex CBC.

foveicollis [Acrostomus]. Kuschel 1958a: 232233, f. 1, 2. Santa Cruz Prov: Viedma Lake leg. Wittmer, holot. $\delta^{1}$, allot., $q$, two parat. in GKC; one parat. in MACN.

One parat. pinned, "Sta. Cruz / L. Viedma / 23.2.48 Wittmer" handwr.; "Paratipo" print. on orange paper; "Acrostomus / foveicollis / n. sp. [handwr.] / Kuschel det. 1955 [print.] on white paper. "MACN-En / 10273” print., reverse.

foveifrons [Linoma] Hustache 1951a: 13. Córdoba Prov.: Alta Gracia, from Bruch n 253.

foveifrons [Solenosternus]: Wibmer \& O'Brien 1986: 295.
One synt. on a card, "Alta Gracia. La Granja / Sierras de Cordoba / C. Bruch leg." print.; "Typus" print. on pale green paper; "253" handwr. on pink paper; "Heterosternus / (Linoma) / foveifrons / Hust." handwr. by Bruch, without frame. We add "Linoma / foveifrons / Hustache 1951 / Syntypus" handwr. on red paper. "MACNEn / 9650" print., reverse. Ex CBC.

fricabilis [Opseotapinopus] Kuschel 1945a: 133-135. Buenos Aires Prov.: Necochea, holot. in MLP; Mar del Plata parat.; La Pampa, San Luis Prov., in several repositories.

One parat. pinned, "Prov. Buenos Aires / Col. Mus. L. Pl." handwr. by Bruch; "Opseotapinopus / fricabilis / Kschl. / Paratipo" handwr. on red paper. "MACN-En / 10272" print., reverse.

fuirenae [Sibariops] Bondar 1943: 68-69. Brasil: Bahia: Pitúba III-VIII.1942, on Fuirena umbellata (Cyperaceae), 150 spec. in BON, MZSP, FMNH.

saba [Neomadarus] (Fabricius 1801): Kuschel 1983: 40. = Sibariops fuirenae Bondar 1943, = Limnobaris boliviensis Hustache 1924.

fuirenae [Sibariops]: Vaurie 1953: 26-27. Designates lectot. ex 16 'cot.'; other 'cot.' in FMNH, MZSP.

One paralect. on a card, "Pitúba, nos arredores Bahia", "Estado da Bahia III-VIII. Brazil. G. Bondar leg.", "on Fuirena umbellata" print.; "Cotype / Sibariops / fuirenae / Bond." print. on red paper. "MACN-En / 10271" print., reverse.

fulvosuturalis [Conotrachelus] Hustache 1926: 244, 253, pl. IV: f. 9. Buenos Aires Prov., from Bruch.

Two synt. on cards, on separate pins, "Rep. Argentina / Prov. Buenos Aires / 3.1896 [190

] / C. Bruch" print., date handwr., black frame; "Typus" on pale green paper. One of them also "Conotrachelus / fulvosuturalis / Hust." handwr. by Bruch, red frame. The other one also "Foto" on pale green paper; we add "Conotrachelus / fulvosuturalis / Hustache 1926 / Syntypus" handwr. on red paper. "MACN-En / 10097 [10098]" print., reverse. Ex CBC.

funebris [Lydamis] Hustache 1950a: 27. In a key; Brasil: Jatahy.

semiluctuosus [Lydamis] (Blanchard 1847): Kuschel 1955: 274. = L. funebris Hustache.

Two synt. pinned, "Jatahy / Brasil" handwr. by Bruch; "Typus" ["Cotypus"] handwr. by Bruch on pale green paper; "Lydamis / funebris / Hust." 
handwr. by Bruch, without frame. The "typus" also "Lydamis / funebris m." handwr. by Hustache?, cut. "MACN-En / 9545 [9546]" print., reverse. Ex $\mathbf{C B C}$.

funicularis [Acyphus] Heller 1921: 27, f. 6. Santa Fe Prov., from Bruch.

renggeri [Acyphus] (Labram \& Imhoff 1849): Wibmer \& O'Brien 1986: 52. = A. funicularis Heller, fide Kuschel.

One synt. pinned, "Rep. Argentina / Prov. Santa Fe / V.1913 / C. Bruch" print., date handwr., black frame; "Rosario / Hubrich leg." handwr. by Bruch; "Typus" print. on pale green paper; "Acyphus / funicularis / Heller" handwr. by Bruch, red frame. "MACN-En / 10011" print., reverse. Ex $\mathbf{C B C}$.

garciai [Tachygonus] Viana 1954: 173-177, f. 13, 14. Paraguay: Itapuami, leg. Martínez 30.XII.1950, holot. $\delta$, allot, + , two parat. ${ }^{\top}$, ㅇ, in MACN $n^{\circ}$ 53017, other parat. in AMC, MVC.

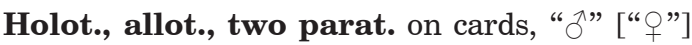
print.;"Paraguay / Itapuami / A. Martínez / 30XII-1950 [on one 30-XI-1950]" handwr.; "53017" handwr.; "Holotypus" ["Allotypus", "Paratypus"] print. on red [red, yellow] paper; "Tachygonus / garciai / Viana / Holotipo o [Alotipo $\odot$, Paratipo o, Paratipo o] [handwr.] / Museo Argentino de Ciencias Naturales [print.]" on red [red, yellow, yellow] paper. The holot. also "Dibujado" (depicted) [handwr.]. One slide "Antena. Paratipo. ejem. Paraguay. Itapuami. Leg. A. Martínez 30X-1950", "Tachygonus garciai Viana X-1951". In slide coll. sub T84. "MACN-En / 10391 (holot.) [10392 (allot.), 10393, 10394 (both parat.)]" print., reverse.

Note: One $\widehat{\delta}$, two 9 parat. are at MLP (Morrone \& Loiacono 1994: 21).

gemignanii [Amalactus] Viana 1951: 32, 3943, f. 6, 7, pl. III. Chaco Prov.: Resistencia, leg. Daguerre XII.1935, holot. $\hat{\alpha}$, allot. $\odot$, two parat. $\delta$ in MACN $n^{\circ} 40122$.

aterrimus [Amalactus] Boheman 1843: Kuschel 1955: 272. = A. gemignanii .

Holot., allot., two parat. pinned, the holot.

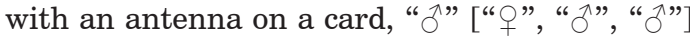
print.; "Chaco - Argentina / Dep. Resistencia. X-XII-935 / J.B. Daguerre" print.; "Holotypus" ["Allotypus", "Paratypus"] print. on red [red, yellow] paper; "Amalactus gemignanii / Viana / Holotipo $\widehat{\partial}$ [Alotipo 9 , Paratipo $\delta^{\lambda}$ [ [handwr.] / Museo Argentino de Ciencias Naturales [print.]” on red [red, yellow] paper. "MACN-En / 10409 (holot.) [10410 (allot.), 10411, 10412 (both parat.)]" print., reverse.

gemignanii [Cylydrorhinus] Kuschel 1958a: 243-244, f. 14, 15. Santa Cruz [Prov.] leg. Mateo Gómez, holot. in MACN.

Holot. on a card, "Holotipo" print. on orange paper, black frame; "Argentina / Sta. Cruz" print., black frame; "Cylydrorhinus / orfilai / n. sp. [handwr.] / Kuschel det. 1955 [print.]" on white paper; "Cylydrorhinus / gemignanii / n. sp.[handwr.] / det. G. Kuschel / 1955 [print.]" on white paper. "MACN-En 10367" print., reverse. Note: The spec. is labeled "orfilai" in addition to "gemignanii", rotulation error?

geminatus [Hyperodes] Hustache 1926: 202, 206, pl. III: f. 1. Buenos Aires Prov., from Bruch. geminatus [Listronotus]: O'Brien 1979: 267.

Two synt. on cards, on separate pins, "Rep. Argentina / Prov. Buenos Aires / 190_/ C. Bruch" print., black frame; "Typus" print. on pale green paper. One of them also "Hyperodes / geminatus / Hust." handwr. by Bruch, red frame; "Hyperodes / geminatus" handwr. by Hustache?, cut; "149" handwr. on pink paper. The other one also "Foto" print. on pale green paper; we add "Hyperodes / geminatus / Hustache 1926 / Syntypus" handwr. on red paper. "MACN-En / 9467 [9468]" print., reverse. Ex CBC.

glaber [Conotrachelus] Hustache 1926: 247, 254. Buenos Aires Prov.: La Plata; Rio Santiago, from Bruch and Lizer.

glaber [Conotrachelus]: Wibmer \& O'Brien 1986: 161. = aequalis Fiedler 1944 non Fiedler 1940, fide Kuschel 1950a: 18.

Nine synt. on cards, on four pins $(1+2+3+3)$, "Rep. Argentina / Prov. Buenos Aires / 24.V.1903 [190_, 190_, 190_]/ C. Bruch" print., date handwr., black frame; "Cotypus" print. on pale green paper; "Conotrachelus / glaber / Hustch. [Hust., Hust.] handwr. by Bruch, red frame [without frame, without frame, without frame]. "MACNEn / 10119 [10120 through 10127]" print., reverse. Ex CBC.

glaberrimus [Cylydrorhinus] Kuschel 1958a: 242-243, f. 12, 13. Chubut Prov.: Esquel; "Patagonia", Neuquen Prov. Chile: Aysén. Holot. $\delta^{\lambda}$, allot. $\rho$ in MNNC, parat. in several repositories, including MACN.

One parat. pinned, " 7926 " handwr.; "Rio Negro" handwr. on green paper, reverse white; 
"55." handwr.; "Paratipo" print. on pale yellow paper; "Cylydrorhinus / glaberrimus / n. sp. [handwr.] / Kuschel det. 1957 [print.]" on white paper. "MACN-En / 10281" print., reverse. Note: Two ${ }^{\lambda}$, four $\odot$ parat. are at MLP (Morrone \& Loiacono 1994: 11).

glabrescens [Tyloderma] Wibmer 1989: 18, 4647, f. 31, 52, 94, 131. Bolivia: Santa Cruz, $4 \mathrm{mi}$ E of S. Cruz (type locality), holot. in CWOB; Argentina: Corrientes and Formosa Prov.; Bolivia. Paraguay. Parat. in several repositories, including MACN.

Six parat. on cards, on three pins $(1+1+4[4=$ $3+1]$ ), the first two pins clearly separated from the third one, and remounted on pointed cards, "S. Tomé / Corrientes / 10.928" handwr. at the " $1+1$ ", and print. with date handwr. at the " 4 $(=3+1)$ "; "Paratype / Tyloderma / glabrescens / Wibmer 1989" print. on yellow paper. "MACN-En / 10236 [10237 through 10241" print., reverse.

Note: One parat. is at MLP (Lanteri, Suárez \& del Río 2003).

goyazensis [Heterosternus] Hustache 1951a: 23. Brasil: Goyaz: Jatahy (types, from Donckier); Mato Grosso [do Sul]: Corumbá.

[goyazensis] [Heterothorax] Marshall 1946: 97. Nom. nov. pro Heterosternus Kirsch 1870 non Dupont 1832.

[goyazensis] [Solenosternus] Kuschel 1983: 42. Heterothorax Marshall $=$ Solenosternus Schoenherr 1826.

One synt. on a pointed card, "Jatahy / Brasil" handwr. by Bruch; "Typus" handwr. by Bruch on pale green paper; "Heterosternus / Goyazensis / Hust." handwr. by Bruch, without frame; "Heterosternus / Goyazensis m." handwr. by Hustache?, cut."MACN-En / 9638" print., reverse. $E x$ CBC.

grandis [Brachybaris] Hustache 1951b: 65. Córdoba Province from Bruch n ${ }^{\circ} 238$; Corrientes Prov., Buenos Aires Provinces from Bosq; Chaco de Santiago del Estero: Rio Salado. Uruguay: Montevideo from Lecoq; Cerro Largo 15.XI.1929 leg. Montoro.

[grandis] [Microrhinus]: Kuschel 1950a: 19. Microrhinus = Brachybaris.

One synt. on a pointed card, "Rep. Argentina / Prov. Cordoba / 1[?].1899 / C. Bruch” print., date handwr., black frame; "Brachybaris / grandis / Hust." handwr. by Bruch, without frame; "Brachybaris / grandis m." handwr. by Hustache?, cut. One synt. on a card, "Alta Gracia. La Granja
/ Sierras de Córdoba / C. Bruch leg." print. Both: "Typus" handwr. by Bruch on pale green paper; "238" handwr. on pink paper, "Brachybaris / grandis / Hust." handwr. by Bruch, without frame [green frame]. "MACN-En / 9601 [9602]" print., reverse. Ex CBC.

grandis [Pseudobaris] Hustache 1951a: 18-19. Misiones Prov. from Bruch $n^{\circ}$ 255. Paraguay: Hohenau.

One synt. pinned, "Bompland / Jörgensen" print. One synt. pinned, "Misiones" print.; "255" handwr. on pink paper; "Pseudobaris / grandis m." handwr. by Hustache?, cut. Both: "Pseudobaris / grandis / Hust." handwr. by Bruch, without frame. One synt. on a card, "Paraguay" handwr. by Bruch; we add "Pseudobaris /grandis / Hustache 1951 / Syntypus" handwr. on red paper. All three: "Typus" handwr. by Bruch on pale green paper. "MACN-En / 9635 [9636, 9637]" print., reverse. Ex CBC.

Note: Bompland is a locality in Misiones Prov.

granulospinosus [Heilipus] Brèthes 1920: 4041. Perú: Chanchamayo.

bellicosus [Heilipus] (Herbst 1797): Kuschel 1950b: 112 . = granulospinosus Brèthes, $=b i$ dentatus Guérin

bellicosus [Heilipodus]: Kuschel 1955: 295.

One synt. on a card, remounted (it has a pinhole), "C.R. Vigil / Pérou" handwr. on whitish paper; "Repreparo / M. Viana / I-1960" handwr.; "Heilipus / granulospinosus / Brèthes" handwr. by Brèthes on whitish paper. We add "Syntypus" print. on red paper. "MACN-En / 10334" print., reverse. Ex JB.

gravidus [Cylindrorrhinus] Burmeister 1879: 208-209. Argentina: Northern Patagonia, between Chubut and Negro Rivers.

gravidus [Cylydrorhinus]: Blackwelder 1947: 812.

gravidus [Caneorhinus]: Kuschel 1952c: 121.

Two synt. pinned, one of them " 4544 " handwr.; "Chubut / Valle del Lago Blanco" handwr. on green paper. The other one without labels. We add "Cylindrorrhinus / gravidus / Burmeister 1879 / Syntypus" handwr. on red paper. "MACNEn / 10193 [10194]" print. reverse. Ex HBC.

griseomaculatus [Archopactus cyphoides var.] Hustache 1923: 280. Chaco de Santiago del Estero: Rio Salado in HUS.

griseomaculatus [Naupactus cyphoides var.]: Dalla Torre, Emden \& Emden 1936: 18. 
cyphoides [Naupactus] Heller 1921: Ward, O’Brien, O’Brien, Foster \& Huddleston 1977: 24 = griseomaculatus.

cyphoides [Naupactus]: Wibmer \& O'Brien 1986: 59 . = griseomaculatus, $=$ prasinus Hustache 1947 = viridimarginalis Hustache 1947.

One synt. pinned, "Sgo. del Estero / Rio Salado / Wagner col."; "Cotypus" print. on pale green paper; "Archopactus / griseomaculatus / Hustch." handwr. by Bruch, red frame. "MACN-En / 10055" print., reverse. Ex CBC

Notes: The type should be in HUS. There is another spec. labeled "Cotypus" from Catamarca Prov., not mentioned in the publication, see the third list below.

griseus [Hyperodes] Hustache 1926: 202, 205. Chaco de Santiago del Estero: Rio Salado, leg. Wagner, a series; Buenos Aires Prov., from Bruch.

griseus [Listronotus]: O'Brien 1979: 267.

One synt. on a card, "République Arg.ne / Chaco de Santiago / Del Estero / Rio Salado" print.; "Typus" print. on pale green paper; "Col. / C. Bruch" print., black frame; "Hyperodes / griseus / Hust." handwr. by Bruch, red frame; "yperodes griseus" handwr. by Hustache? cut. "MACN-En / 9469" print., reverse. Ex CBC.

griseus [Torcus] Hustache 1939: 120-121. Buenos Aires Prov., type 10.X:1906, from Bruch $\mathrm{n}^{\circ}$ 128; from Bosq ; Tigre, leg. Viana; Entre Ríos Prov.: Paraná, from Bosq.

One synt. on a card, "Rep. Argentina / Prov. Buenos Aires / 10.X.1906 / C. Bruch” print., date handwr., black frame; "Typus" handwr. by Bruch on pale green paper; "Torcus / griceus (sic) / Hust." handwr. by Bruch, green frame. "MACNEn / 9603" print., reverse. Ex CBC.

Note: Five synt. are at MLP (Morrone \& Loiacono 1994: 25). Tigre refers to the frontal (lower) portion of the Paraná River Delta.

haywardi (subHaywardi)[Crematogasterobius] Hustache \& Bruch 1936: 334-337, f. 1-6, pl. 3: f. 1-5, 7-12. Entre Ríos Prov.: Concordia, Agric. Exper. Stat., on bark of Tipuana tipu (Leguminosae); with the ant Crematogaster brevispinosa Mayr.

Six synt. on cards, on three pins $(1+2+3)$, each pín with an ant on a card. "Concordia / Entre Ríos" handwr. by Bruch; "Typus" ["Typus", "Cotypus"] handwr. by Bruch on pale green paper; "Crematogasterobius / Haywardi / Hust. et Bruch" handwr. by Bruch on white paper, re- mainings of a black frame. "MACN-En / 9674 [9675 through 9679]" print., reverse. Ex CBC.

Note: Three synt. are at MLP (Morrone \& Loiacono 1994: 28-29).

helleri (sub Helleri) [Oxycorynus] Bruch 1912: 266-267, 1 f. Santiago del Estero Prov.: Aerolito, leg. Aula.

helleri [Hydnorobius]: Kuschel 1959b: 269.

One synt. pinned, "Rep. Argentina / Pr. Santiago d. Estero / 190 / C. Bruch" print., black frame; "Aerolito / Aula. leg." handwr. by Bruch; "Typus" print. on pale green paper; "Oxycorynus / Helleri / Bruch" handwr. by Bruch, red frame; "Hydnorobius / helleri / (Bruch) [handwr.] / det. G.Kuschel / 1992 [print.]" on white paper. "MACN-En / 10593" print., reverse. Ex CBC.

hercules [Cylydrorhinus] Kuschel 1958a: 246247, f. 17. Chubut Prov., holot. $\delta$ in MLP, allot. in MACN.

Allot. pinned, "Rep. Argentina / Gob. Chubut / 190_ / C. Bruch" print., black frame; "Alótipo" print. on orange paper; "Cylydrorhinus / hercules n. sp. / Allotypus [handwr.] / Kuschel det. 1957 [print.]" on white paper. "MACN-En 10291" print., reverse.

Note: The holot. is at MLP (Morrone \& Loiacono 1994: 11).

heteropunctatus [Conotrachelus] Hustache 1926: 245, 253. Buenos Aires: La Plata; Rio Santiago, from Bruch \& Bosq.

heteropunctatus [Conotrachelus]: Wibmer \& O'Brien 1986: 161. = pollux Hustache 1939, fide Kuschel 1955: 286.

Three synt., one of them pinned (the pin got broken and we remounted it on a card), the other two on cards, "Rep. Argentina / Prov. Buenos Aires / 190_ [19.XI.1904, 18.IX.1904] / C. Bruch" print., dates handwr., black frames; "Cotypus" ["Typus", "Cotypus"] print. on pale green paper. One of these also "Conotrachelus / heteropunctatus / Hust." handwr. by Bruch, red frame; "C. heteropunctatus m." handwr. by Hustache?, cut; " 20 " handwr. on pink paper. To the other two we add "Conotrachelus / heteropunctatus / Hustahe 1926 / Syntypus" handwr. on red paper. "MACN-En / 10094 [10095, 10096]" print., reverse. Ex CBC.

heterothorax [Enoplopactus] Heller 1921: 25-26, f. 4. Santiago del Estero and Catamarca Prov., $\circ$ from Bruch.

ortizi (sub Ortizi) [Enoplopactus] (Blanchard 
1891): Hustache 1947b: 30.=E. heterothorax. One synt. pinned, "Rep. Argentina / Pr. Santiago d. Estero / 190_ / C. Bruch" print., black frame; "Typus" print. on pale green paper; "Enoplopactus / heterothorax / Heller" handwr. by Bruch, red frame; "Enoplopactus / heterothorax" handwr. by Heller?, cut. "MACN-En / 10057" print., reverse. Ex CBC.

hirsutipennis [Polpones] Hustache 1951b: 42. = Polponella hirsutipennis in litteris. Corrientes Prov.: San Roque leg. Bosq I.1920, types. Paraguay: Asunción.

hirsutipennis [Methyorrhina]: Kuschel 1983: 40.

One synt. on a pointed card, "San Roque / Corr. Bosq" handwr. by Bruch; "Polponella / hirsutipennis / Hust." handwr. by Bruch, without frame; "Polponella / hirsutipennis m." handwr. by Hustache?, cut. We add "Polpones / hirsutipennis / Hustache 1951 / Syntypus" handwr. on red paper. "MACN-En / 10025" print., reverse. Ex CBC.

hirtellus [Brachygeraeus] Hustache 1950b: 4-5. Tucumán Prov. 9 .

[hirtellus] [Eucalus]: Bondar 1950: 472-473. [hirtellus] [Linogeraeus]: Kuschel 1983: 38.

One synt. on a card; an empty card; "Typus" handwr. by Bruch on pale green paper; " 264 " handwr. on pink paper; "Brachygeraeus / hirtellus / Hust." handwr. by Bruch, without frame; "Brachygeraeus / hirtellus m." handwr. by Hustache?, cut. "MACN-En / 9670" print., reverse. $E x$ CBC.

horridus [Cylindrorrhinus] Burmeister 1879: 208. [Santa Cruz Prov.]: Santa Cruz Stream horridus [Cylydrorhinus]: Blackwelder 1947: 812.

Two synt. pinned, one of them "Cylindrorrh. / horridus / Bur. Tipus (in lit.?) [handwr.] / Museo Argentino de Ciencias Naturales [print.]" on white paper, black frame. The other one " 6 " print. on green paper. We add "Cylindrorrhinus / horridus / Burmeister 1879 / Syntypus" handwr. on red paper. "MACN-En / 10207 [10208]" print., reverse. Ex $\mathbf{H B C}$.

humeridens [Trichocyphus] Hustache 1926: 162-163, pl. I: f. 5. Tucumán: Bañado, leg. V. Weiser, two spec.

humeridens [Priocyphus]: Hustache 1939: 45. humeridens [Priocyphopsis]: Lanteri 1990a: 416. Type species of the new genus.

One synt. pinned, abdomen on another card, a plastic microvial with dissected parts; "Typus", "Foto" print. on pale green paper; "Bañado / Tucum. V.921" handwr. by Bruch; "Trichocyphus / humeridens / Hust." handwr. by Bruch, red frame; "Priocyphopsis / humeridens / (Hustache) / det. Lanteri 90" handwr. on white paper. "MACN-En / 10083" print., reverse. Ex CBC.

hustachei [Tyloderma] Wibmer 1989: 17, 2629 , f. $5,42,86,132 .=T$. carinicollis Hustache in sched., nomen nudum. Brasil: Mato Grosso: Rondonopolis, leg. Alvarenga 1975, holot. Brasil, Colombia, Costa Rica, Panamá, Venezuela, in several repositories, including MACN.

Two parat. on pointed cards, on separate pins, "Piracicaba / São Paulo Brazil / XI-13-1965 / C.A. Triplehorn" print.; "Paratype / Tyloderma / hustachei / Wibmer 1989" print. on yellow paper; "ex CWOB" handwr. "MACN-En / 10242 [10243]" print., reverse.

Note: Two parat. are at MLP (Lanteri, Suárez \& del Río 2003).

hylula [Naupactosis] Heller 1921: 27-28, f. 7. Catamarca Prov., from Bruch.

[hylula] [Enoplopactus]: Kuschel 1945a: 122. Enoplopactus $=$ Naupactosis.

One synt. pinned, "Rep. Argentina / Prov. Catamarca / 190 / C. Bruch" print., black frame; "Typus" print. on pale green paper; "Naupactosis / hylula / Heller" handwr. by Bruch, red frame; "Naupactosis / hylula m." handwr. by Heller?, cut. "MACN-En / 10082" print., reverse. Ex CBC.

hypocrita (sub hypocritus) [Geraeus] Hustache 1924: 94-95. Bolivia: Villa Montes XI.1917, types; Santa Cruz IX.1917; Yacuiva VIII.1917; Cuatro Ojos IX.1917; "var. à fond jaune Lagunillas XI.1917, Yacuiva VIII.1917” several spec.

hypocritus [Brachygeraeus]: Hustache 1938a: 101.

[hypocritus] [Eucalus]: Bondar 1950 : 472473.

bellulus [Linogeraeus] (Casey 1922): Kuschel 1983: 38. = hypocritus Hustache, lapsus pro hypocrita; = pergratus (Casey 1922).

One synt. on a card, "Santa Cruz / Bolivia / IX.1917" print.; "Typus" handwr. by Bruch on pale green paper; "Brachygeraeus / hypocrita / Hust." handwr. by Bruch, without frame; "Brachygeraeus / hypocrita m." handwr. by Hustache?, cut. We add "Geraeus / hypocritus / Hustache 1924 / Syntypus" handwr. on red paper. "MACN-En / 9671" print., reverse. Ex CBC. 
hypocritus [Listroderes] Hustache 1929: 197, 200. Chaco de Santiago del Estero: Rio Salado, leg. Wagner; Misiones Prov.: San Ignacio, leg. Wagner; Mendoza Prov., leg. Lizer y Trelles; Buenos Aires Prov., from Bruch and from Bosq. Two synt., one on a card, one pinned, "Rep. Argentina / Prov. Buenos Aires / 190_ [189_] C. Bruch" print., black frame; "Typus" print. on pale green paper; "Listroderes / hypocritus / Hust." handwr. by Bruch, red frame; "istroderes [List.] / hypocritus [m.]" handwr. by Hustache?, cut., the 2nd one also "208" handwr. on pink paper. "MACN-En / 9699 [9700]" print., reverse. Ex CBC.

insuetus [Machaerophrys] Kuschel 1958a: 237238, f. 7. Chubut Prov., holot. $\circ$ in MACN.

Holot. on a card, "Holotipo" print. on orange paper, black frame; "Chubut" handwr. on pale green paper, black frame; "Machaerophrys / insuetus / n. sp. [handwr.] / Kuschel det. 1955 [print.]" on white paper. "MACN-En / 10348" print., reverse.

jekeli [Lordops] Heller 1921: 31 = jekeli Faust in litteris. Santiago del Estero Prov., from Bruch.

One synt.? pinned, "Rep. Argentina/Pr. Santiago d. Estero /190_ / C. Bruch" print., black frame; "Lordops / Jekeli / Heller" handwr. by Bruch, red frame; "Lordops Jekeli / Det. K.M.H." handwr. by Heller?, cut. We add "Syntypus?" handwr. on red paper. "MACN-En / 10163" print., reverse. Ex CBC.

Note: The spec. is not labeled as a type, setting doubt about its condition.

jujuyensis [Cratosomus (Eucratosomus) sticticus subsp.] Kuschel 1945b: 366-367, f. 1b, 3, photo 3. Jujuy Prov. Holot. in MACN.

granifer [Cratosomus sticticus subsp.] Emden 1933: Wibmer \& O'Brien 1986: 259. = jujuyensis.

Holot. pinned, "7274" handwr.; "Jujuy" handwr. on green paper, reverse white; "Cratosomus / sticticus / jujuyensis / Kschl. Tipo" handwr. on red paper; "Cratosomus / (Eucrat.) / sticticus / jujuyensis Kschl. / K45. Tipo" handwr. on white paper, frame as a graeca. "MACN-En / 10337" print., reverse.

kuscheli [Tachygonus] Viana 1954: 151-152, f. 1, 2. Bolivia: Coroico: Archeta $1800 \mathrm{~m}$, leg. Kuschel 14.II.1948. Holot. in GKC.

A slide "Antena. Tipo. Bolivia - Coroico. Archeta 1800 m. Leg. Kuschel II.1948 [handwr.] / Museo
Arg. C. Naturales Sección Entomología [print.]". "MACN-En / 10569" print., reverse. In slide coll. sub T85.

lacordairei [Tyloderma] Wibmer 1989: 18, 4546, f. 51, 93, 129. Bolivia: Santa Cruz, $10 \mathrm{mi} \mathrm{W}$ of Puerto Banegas, type local., in CWOB; Bolivia. Paraguay. Argentina: Buenos Aires, Corrientes, Formosa, Misiones, Santa Fe, Tucumán Prov.; parat. in several repositories, including MACN. One parat. on a pointed card, "Buen. / Aires" print. on green paper. Two parat. on cards, "Argentina [print.] / Quilmes [handwr.] / Bs. Aires [print.]" black frame; "33970" handwr. (in register book, also "Excursión Daguerre, De Carlo, Viana"): One parat. on a card, "Argentina [print.] / Delta [handwr.] / Buenos Aires [print.]"; "34560" handwr. (in register book also "18-19III-1934, Doello, Daguerre, Viana”). All four: "Paratype / Tyloderma / lacordairei / Wibmer 1989" print. on yellow paper. "MACN-En / 10213 [10214, 10215, 10216]" print., reverse.

Note: Three parat. are at MLP (Lanteri, Suárez \& del Río 2003).

lactifer [Cylindrorrhinus] Burmeister 1879: 206-207. Santa Cruz Prov.: Santa Cruz and Chico Streams.

lactifer [Cylydrorhinus]: Blackwelder 1947: 812.

lactifer [Cylydrorhinus]: Wibmer \& O'Brien 1986: 105. = Otideres externevittatus Fairmaire 1884, = Otidoderes quadrilineatus Bovie 1907, fide Bruch 1921.

Four synt. (all four?) pinned. The 1st \& 2nd ones without labels; the 3rd one " 25 " print. on green paper; the 4th one "Cylindrorrh. / latifer / Burm. Tipo [handwr.] / Museo Argentino de Ciencias Naturales [print.]" on white paper, black frame. We add "Cylindrorrhinus / lactifer / Burmeister 1879 / Syntypus" handwr. on red paper. "MACN-En / 10199 [10200, 10201, 10202]" print., reverse. Ex HBC.

laevipennis [Cylydrorhinus] Kuschel1958a:244245, f. 16. Chile: Aysén: Balmaceda. Argentina: Chubut \& Neuquen Prov., "Patagonia". Holot. $\uparrow$, allot. $\curvearrowright$, parat. in GK; parat. in several repositories, including MACN, MLP.

One parat.pinned, "10264" handwr.; "Neuquen" handwr. on green paper, reverse white; " 24 " handwr. One parat. pinned, "Patagonia / Col. Mus. L.P." handwr. by Bruch; "Cylindrorhinus / biramosus / Bruch [handwr. by Bruch] / C. Bruch determ. [print.]" on white paper. Both: "Parátipo" 
print. on pale yellow paper; "Cylydrorhinus / laevipennis / n. sp. [handwr.] / Kuschel det. 1957 [print.]" on white paper. "MACN-En / 10284 [10285]" print., reverse.

Note: One $\delta$ parat. is at MLP (Morrone \& Loiacono 1994: 11).

lateralis [Centrinaspis] Hustache 1950b: 22. Tucumán Prov., from Bruch n 263.

perscitus [Linogeraeus] (Herbst 1797): Kuschel 1983: 39. = connivens $($ Casey 1920), = exulans (Casey 1892), = lateralis $($ Hustache 1950), = regressa (Casey 1920).

perscitus [Linogeraeus] (Herbst 1797): Wibmer \& O’Brien 1986: 309, = connivens (Casey 1920), = exulans (Casey 1892), = lateralis (Hustache 1950), = marginatus: Champion $1908,=$ peras cites Pierce 1915: Hustache 1938, = regressa (Casey 1920).

One synt. on a pointed card, "Rep. Argentina / Prov. Tucuman / 19.I.1904 / C. Bruch" print., date handwr., black frame; "Typus" handwr. by Bruch on pale green paper; " 270 " handwr. on pink paper; "Centrinaspis / lateralis / Hust." handwr. by Bruch on white paper, without frame; "Centrinaspis / lateralis m." handwr. by Hustache?, cut. "MACN-En / 9673" print., reverse. $E x$ CBC.

lateralis [Conotrachelus] Brèthes 1910: 220. Bolivia.

brethesi [Conotrachelus] Hustache 1926: 254. Nom. nov. pro C. lateralis Brèthes 1910 non Champion 1904.

One synt. on a card, remounted, "Bolivia" handwr. on green paper, reverse white; "Repreparo / M. Viana / I-1960" handwr.; "10241" handwr.; "Conotrachelus / lateralis / Brèthes" handwr. by Brèthes on whitish paper. We add "Syntypus" print. on red paper. "MACN-En / 10364" print., reverse. Ex JB.

lembulus [Ilyodytes] Kuschel 1952a: 68-69, f. 45. Buenos Aires Prov.: Ituzaingó, holot. $\delta$ in MACN $n^{\circ} 7517$.

Holot. on a pointed card, "7517" handwr.; "ㅇ" print.; "Ituzaingó" handwr. on green paper, reverse white; "Holotipo" print. on orange paper; "Ilyodytes / lembulus / Kuschel [handwr.] / Kuschel det. 1959 [print.]" on white paper. "MACN-En / 10355" print., reverse.

lineatula [Antesis] Hustache 1951b: 35. "= puncticollis m. in litt." Santa Fe Prov.; Entre Ríos Prov.: Paraná; Córdoba Prov., ex Bruch nº 272.
One synt. on a pointed card, "Alta Gracia. La Granja / Sierras de Córdoba / C. Bruch leg." print.; "Typus" handwr. by Bruch on pale green paper; "272" handwr. on pink paper; "Antesis / lineatulus / Hust." handwr. by Bruch, without frame; "Antesis / lineatulus m." handwr. by Hustache?, cut. "MACN-En / 9606" print., reverse. $E x$ CBC.

lineatulus [Centrinites] Hustache 1950b: 123. Brasil: Espirito Santo State, leg. Bang-Haas. seven spec.

One synt. on a tiny pointed card, "Espir. Santo / Brasil" handwr. by Bruch; "Typus" handwr. by Bruch on pale green paper; "Centrinites / lineatus (sic) / Hust." handwr. by Bruch, without frame; "Centrinites / lineatulus m." handwr., cut. "MACN-En / 9547" print., reverse. Ex CBC.

litus [Helodytes (Dythelus)] Kuschel 1952a: 5658, f. 39, 40. Misiones Prov. $\mathrm{n}^{\circ}$ 51370, ex ABC, one parat in MACN. Bolivia: Beni: Trinidad 31.XII.1948, 2.I.1949, on aquatic Gramineae, holot., allot., forteen parat. in GK. Misiones Prov., $n^{\circ} 51370$, ex ABC, one parat in MACN. Parat. in several repositories.

One parat. on a card, "Rep. Argentina / A. Breyer" print., with color stripes indicative of Misiones Prov.; "Argentina / Misiones" print.; "51370" handwr.; "Paratipo" print. on orange paper; "Helodytes / litus n. sp. [handwr.] / Kuschel det. 1951 [print.]" on white paper. "MACN-En / 10254" print., reverse.

longimanus [Gasterocercus] Brèthes 1910: 220. Misiones Prov.

longimanus [Episcirrus]: Kuschel 1958b: 762. One synt. on a card, remounted, it has a pinhole, "Misiones" handwr. on green paper, reverse white; "Repreparo / M. Viana / I-1960" handwr.; "10258" handwr.; "Gasterocercus / longimanus Brèthes" handwr. by Brèthes on whitish paper. We add "Syntypus" print. on red paper. "MACNEn / 10347" print., reverse. Ex JB.

longisquameum [Tyloderma] Wibmer 1989: 20, 80-81, f. $12,70,112$, 131. Santa Fe Prov.: San Javier: Colonia Macías, Holot. in MACN. Buenos Aires, Santa Fe Prov., parat. in several repositories.

Holot., allot. on pointed cards, "ㄱ" [" $\bigcirc$ "] print.; "Arg. P. Santa Fe / Colonia Mascias / Dept. San Javier Dec.1960 / M. Viana" print., date handwr.; a plastic microvial with dissected parts; "Holotype [Allotype]/Tyloderma/longisquameum/Wibmer 1989" print. on red paper. One parat. on a card, 
"Col. / A. Breyer" print.; "Paratype /Tyloderma / longisquameum / Wibmer 1989" print. on yellow paper. "MACN-En / 10246 (holot.) [10245 (allot.), 10244 (parat.)]” print., reverse.

longulus [Amathynetes] Kuschel 1949: 49-51, f. 7a-c. Chile: Arica $4400 \mathrm{~m}$; Cotacotani Lake 4400 m; Tacora: Aguas Calientes 4400 m. Holot., one parat. in MNS, parat. in several repositories, including MACN, MLP.

[longulus] [Puranius]: Kuschel 1955: 288. Amathinetes $=$ Puranius.

longulus [Macrostyphlus]: Wibmer \& O’Brien 1986: 117.

longulus [Amathynetoides]: Morrone 1994a: 32. One parat. on a pointed card, "Parátipo" handwr. on orange paper; "Arica. Parinacota / $4500 \mathrm{~m}$ 1-3-48" handwr.; "52480" handwr.; "Amathynetes / longulus / Kschl. / Kuschel 1949" handwr. on white paper, black frame. "MACN-En / 10249" print., reverse.

luteiventris [Perisochoinus, sic, pro Parisoschoenus] Hustache 1924: 111-112. Bolivia: Santa Cruz IX.1917, type. Several spec. luteiventris [Nicentrus]: Kuschel 1983: 40.

luteiventris [Nicentrus]: Wibmer \& O'Brien 1986: 316. Perisochoinus Hustache and Perosochoinos Hustache are lapsus pro Parisoschoenus Faust 1896.

One synt. on a pointed card, "Santa Cruz / Bolivia" print.; "Typus" handwr. by Bruch on pale green paper; "Nicentrus / luteiventris / Hust." handwr. by Bruch, without frame; "Nicentrus / luteiventris m." handwr. by Hustache?, cut. We add "Perisochoinus / luteiventris / Hustache 1924 / Syntypus" handwr., on red paper. "MACNEn / 9688" print., reverse. Ex CBC.

luteolus [Torcus] Hustache 1939: 118. Buenos Aires IV.1905, Bruch n 126; Bosq n 102; Luján; Tigre; San José.

One synt. on a card, "Flores. B. As. / IX:918. Bosq" handwr. by Bruch, "Typus" handwr. by Bruch on pale green paper; "Torcus / luteolus / Hust." handwr. by Bruch without frame; "Torcus / luteolus m." handwr. by Hustache?, cut. One synt. on a card, "Rep. Argentina / Prov. Buenos Aires / 10.X.1906 / C. Bruch" print., date handwr., black frame; "126" handwr. on pink paper;"Typus" handwr. by Bruch on pale green paper; "Torcus / luteolus / Hust." handwr. by Bruch, green frame. One synt. on a card, "Bs. Aires (Tigre) (C. Bruch)" handwr. by Bruch; "Fototypus" handwr. by Bruch on pale green pa- per. We add "Torcus / luteolus / Hustache 1939 / Syntypus" handwr. on red paper. "MACN-En / 9611 [9612, 9613]” print., reverse. Ex CBC.

Notes: Seventeen synt. are at MLP (Morrone \& Loiacono 1994: 25). San José de Flores is a district in Buenos Aires City.

magellanicus [Acrostomus] Kuschel 1958a: 233. Chile: Magallanes Prov.: Ultima Esperanza: Guido Mount, 800 m, Cazador Mount, 800 m, on Bolax and Azorella respectively, leg. Kuschel, holot. ${ }^{1}$, allot. + , parat., in GKC, parat. in several repositories, including MACN.

One parat. pinned, "Chile / Magallanes/ $\mathrm{C}^{\circ}$ Castillo. $\mathrm{C}^{\circ}$ Cazador $800 \mathrm{~m}$ 9.2.57" handwr.; "s/ Azorella sp." handwr.; "Coll. Kuschel" print.; "Parátipo" print. on white paper; "Acrostomus / magellanicus / n. sp. [handwr.] / Kuschel det. 1957 [print.]" on white paper. "MACN-En / 10270" print., reverse.

manni [Scotoeborus] Kuschel 1949: 40-41, pl. III: f. 2. Chile: Arica: Cotacotani Lake 4500 m; Lipiche $4500 \mathrm{~m}$; Tacora: Aguas Calientes 4400 m. Holot., parat. in MNNC, parat. in several repositories, including MACN

manni [Cylydrorhinus]: Wibmer \& O'Brien 1986: 105.

One parat. pinned, "Parátipo" handwr. on orange paper; "Arica. Tacora $4800 \mathrm{~m}$ 6-3-48" handwr.; "52481" handwr.; "Scotoeborus / manni / Kschl. / Kuschel 1949" handwr. on white paper, black frame. "MACN-En / 10269" print., reverse.

marginicollis [Hyperodes] Hustache 1926: 202, 209-210, pl. III: f. 4. Buenos Aires, from Bruch and from Bosq.

marginicollis [Listronotus]: O'Brien 1979: 267.

Two synt. on cards, on separate pins, "Rep. Argentina / Prov. Buenos Aires / 190_ / C. Bruch" print., black frame; "Typus" print. on pale green paper. One of them also "Hyperodes / marginicollis / Hust." handwr. by Bruch, red frame; " $\mathrm{n}^{\circ} 131$ Hyperodes / marginicollis m." handwr. by Hustache?, cut, "131" handwr. on pink paper. The other one also "Foto" print. on pale green paper; we add "Hyperodes / marginicollis / Hustache 1926 / Syntypus" handwr. on red paper. "MACN-En / 9470 [9471]" print., reverse. Ex CBC.

marinus [Cylindrorrhinus clathratus var.] Burmeister 1879: 204. [Santa Cruz Prov.]: Santa Cruz Stream, one spec. 
marinus [Cylydrorhinus clathratus var.]: Blackwelder 1947: 812.

murinus (sic) [Cylydrorhinus]: Kuschel 1958a: 241.

Holot. pinned, without labels, features as in original description; we add "Cylindrorrhinus / clathratus / var. marinus / Burmeister 1879 / Typus" handwr. on red paper; "Holotypus" print. on red paper. "MACN-En /10209" print., reverse. Ex HBC.

Note: The paper published by Burmeister in Stettiner entomologische Zeitung 40: 204, housed in the library of the MACN, has been corrected with pencil by Burmeister himself to "murinus".

martinezi [Mesothanius] Kuschel 1950b: 110 111, pl. III: f. 9. Bolivia: La Paz: Sud- Yungas: Ocobaya $1500 \mathrm{~m}$ leg. Martínez 2.I.1949. Holot. , allot. $\delta$, three parat. in GK; parat. in several repositories, MACN not mentioned.

One parat. on a card, "Sud Yungas / Ocobaya. Colaya $1500 \mathrm{mts} / \mathrm{Dep}$. La Paz. Bolivia / A. Martínez coll. / I-49" photographed from typed; "Paratipo" print. on orange paper; "Paratype" handwr. on orange paper; "Mesothanius / martinezi / Kschl. / Kuschel 50" handwr. on white paper. "MACN-En / 10262" print., reverse.

martinezi [Tachygonus] Viana 1954: 155-158, f. 3, 4. Misiones Prov.: Dept. San Javier: Santa María leg. Viana XI-1948, holot. Ô, allot. +, parat. two ${ }^{\lambda}$, one + in MACN n ${ }^{\circ} 53015$. Parat. in AMC, GKC, MVC.

Holot., allot., two parat. on cards, on separate pins, “ㄲ”, [“ㅇ”, “ㄲ”, “ㄲ”] print.; "Misiones. Argentina / Dep. Concep. Sta. María / XI-1948. M.J. Viana" print., date handwr.; "53015" handwr.; "Holotypus" ["Allotypus", "Paratypus"] print. on red [red, yellow] paper; "Tachygonus / martinezi / Viana/ Holotipo ổ [Alotipo $\uparrow$, Paratipo §] [handwr.] / Museo Argentino de Ciencias Naturales [print.]" on red [red, yellow] paper. "MACN-En / 10400 (holot.) [10401 (allot.), 10402, 10403 (both parat.)]" print., reverse. A slide "Antena. Paratipo. ejem: / Argentina. Misiones. Santa María. Leg. M. Viana, Tachygonus martinezi Viana. Det. M. Viana X-1950 [handwr.] / Museo Arg. C. Naturales. Sección Entomología”. In slide coll. sub T87.

melanoleucus [Cylindrorrhinus] Burmeister 1879: 207. [Santa Cruz Province]: Santa Cruz Stream.

melanoleucus [Cylindrorrhinus]: Schenkling \& Marshall 1931: 3. = Otideres cancellatus Fairmaire 1884. melanoleucus [Cylydrorhinus]: Blackwelder 1947: 812. (= cancellatus Fairmaire 1884).

melanoleucus [Cylydrorhinus]: Morrone 1995: 95. $=$ C. melanoleucus aysenensis Kuschel 1958a: 248, = chubutensis Kuschel 1958a: 247.

Four synt., all four? pinned. The 1st one: "4" print. on green paper. The 2nd one: "Cylindrorrh. / melanoleucus / Burm. Tipo [handwr.] / Museo Argentino de Ciencias Naturales [print.]" on white paper, black frame. The 3rd one: without labels. The 4th one: "lineatus / Voy. Pol. Sd." handwr. We add "Cylindrorrhinus / melanoleucus / Burmeister 1879 / Syntypus" handwr. on red paper. "MACN-En / 10203 [10204, 10205, 10206]" print. reverse. Ex HBC.

mendozensis [Baris] Casey 1922: 479. In a key. One synt. on a card, "Hualfin / Catam. Weiser" handwr. by Bruch; "Typus" handwr. by Bruch on pale green paper; "239" handwr. on pink paper; "Baris / mendozensis / Casey" handwr. by Bruch, green frame. "MACN-En / 9630" print., reverse. Ex CBC.

mendozensis [Heilipus vicinus var.] Hustache 1938c: 347: Kuschel 1955: 296.

ventralis [Heilipus] Hustache 1938c: 346: Kuschel 1955: 296.

ventralis [Heilipodus]: Wibmer \& O'Brien 1986: 141. = Heilipus vicinus var. mendozensis.

One synt. pinned, "Rep. Argentina / Prov. Mendoza / 190_ / C. Bruch" print., black frame; "Typus" print. on pale green paper; "Hilipus / mendocensis (sic) / Hust." handwr. by Bruch, red frame; "mendozensis *m." handwr. by Hustache?, cut. "MACN-En / 10162" print., reverse. Ex CBC.

mimica [Bondariella] Hustache \& Bondar 1942: 21-22, f. 4, 7. Brasil: Bahia State, some 400 spec. on $\delta$ flowers of Cocos schizophylla (Palmae), 'cotypes' in several repositories, MACN and MLP not mentioned.

mimica [Bondariella]: Vaurie 1953: 26-27. Designates lectot. ex 123 'cot.'; other 'cot.' in FMNH, IBSP, HUS.

Two paralect. on pointed cards, on separate pins, "nos arredores Bahia" (at the sorroundings of Bahia) print.; "Estado da Bahia XI:1:41. Brazil. G. Bondar leg." print., date handwr.; 253515" handwr.; "Cotype / Bondariella / mimica / H. \& B." print. on red paper. "MACN-En / 10278 [10279]" print., reverse.

Note: Twenty paralect. are at MLP (Morrone \& Loiacono 1994: 25-26). 
minor [Pantomorus (Graphognathus)] Buchanan 1942: 107-108, 110. USA, Florida, Pensacola, I-VIII-1941, USNM 56306, holot. , 230 parat., USA, Florida: Pensacola, González, Cantonment, Bluffs Springs, Century, and Crestview.

minor [Graphognathus]: Buchanan 1947: 21.

minor [Graphognathus]: Wibmer \& O'Brien 1986: 63. = breviscapus (Hustache 1947), fide Kuschel 1950a: 10.

minor [Naupactus]: Lanteri \& Marvaldi 1995: 221.

One parat. on a pointed card, "Pensacola Fla. / Jul-Aug. '41 - 14538 FJB * 4a", "Naval Air Station Tx. clover" handwr.; "Paratype / minor / 56306 USNM" print. and handwr. on red paper; "53486" handwr.; "Pantomorus / (Graphognathus) / minor / Buch. LB '42" handwr. on white paper. "MACNEn / 10305" print., reverse.

Note: Two parat. are at MLP (Morrone \& Loiacono 1994: 7).

minutus [Ovanius] Hustache 1950b: 95-96. Brasil, from Bruch.

One synt. on a pointed card, "Rep. Brasil / 190 / C. Bruch" print., black frame; "Typus" print. on pale green paper; "Ovanius / minutus / Hust." handwr. by Bruch, without frame; "Ovanius / minutus m." handwr., by Hustache? cut. "MACNEn / 9548" print., reverse. Ex CBC.

molitor [Opseotapinotus] Heller 1921: 31. Catamarca Prov. leg. Bruch 10.IV.1908.

One synt.? pinned, "Rep. Argentina / Prov. Catamarca / 29.III.1907 / C. Bruch" print., date handwr., black frame; "Typus" print. on pale green paper; "Opseotapinopus / molitor / Heller" handwr. by Bruch, red frame. One synt.? pinned, "Hualfin / Catam. Weiser" handwr. by Bruch, "Cotypus" handwr. by Bruch on pale green paper; "19289" handwr. (in register book same data); "Opseotapinopus / molitor / Heller" handwr. by Bruch, green frame. "MACN-En / 10066 (typus) [10065 (cotypus)]" print., reverse. Ex CBC.

Note: The collecting date of the first spec. and the locality of the second one, differ from those of the publication, setting doubt about their type condition.

monrosi [Tachygonus] Viana 1954: 166-169, f. 9, 10. Misiones Prov.: Loreto, leg. Ogloblin, holot $\rho$ in MACN n ${ }^{\circ} 53016$.

Holot. on a card, "†" print., "Dibujado" (depicted) handwr.; "Argentina / Misiones. Loreto / Leg. A.A. Ogloblin" handwr.; "53016" handwr.;
"Prep. N 3043" handwr.; "Holotypus" print. on red paper; "Tachygonus / monrosi / Viana / Holotipo $q$ [handwr.] / Museo Argentino de Ciencias Naturales [print.]" on red paper. One slide "Antena. Holotipo ejem. $\mathrm{N}^{\circ}$ Argentina. Misiones. Loreto. Leg. A.A. Ogloblin. N 3043"; "Tachygonus monrosi Viana. Det. M. Viana X-1950 [handwr.] / Museo Arg. C. Naturales. Sección Entomología [print]". In slide coll. under T86. "MACN-En / 10397" print., reverse.

mordor [Acrostomus] Morrone 1994b: 406, 409-410, f. 8, 9, 17, 21, 23, 26, tab. 1. Neuquen Prov., holot. ${ }^{\top}$, five parat. in MACN n ${ }^{\circ} 10264$; parat. in MLP, AMNH.

Holot., five parat. pinned, holot. with a plastic microvial with dissected parts, abdomen on a card, "Neuquen" handwr. on green paper; "35" handwr.;"10264" handwr. (in registration book dame data, and "leg. Sr. Illin"); "60 [62, 63, 64, 67]" print.; "Acrostomus / mordor Morrone / holotype [paratype] male" print. on red [yellow] paper. "MACN-En / 10330 (holot.) [10325 through 10329 (all parat.)]" print., reverse.

multimaculata [Centrinaspis] Hustache 1950b: 12. Brasil: Goyaz State: Jatahy.

convexus [Linogeraeus] (Hustache 1950): Kuschel 1983: 38. = C. multimaculata Hustache.

One synt. on a card, "Jatahy / Goyaz" print. on whitish paper; "Typus" handwr. by Bruch on pale green paper; "Centrinaspis / multimaculatus / Hust." handwr. by Bruch, without frame; "Centrinaspis / multimaculatus m." handwr. by Hustache?, cut. "MACN-En / 9684" print., reverse. Ex CBC.

natator [Tyloderma] Wibmer 1989: 18, 48-49, f. 24, 53, 95, 133. Uruguay: Colonia: Reducto, 13 km NNE Colonia, type local., holot. in CWOB. Argentina: Buenos Aires, Corrientes, Entre Ríos Prov. Paraguay. Uruguay, parat. in several repositories, including MACN.

Three parat. on cards, on two pins $(1+2)$. The 1st pin: "Entre Ríos / Concordia / Daguerre" print.; "26109" handwr. (in register book same data); "Paratype / Tyloderma / natator / Wibmer 1989" print. on yellow paper. The 2nd pin: "Entre Ríos / Paraná / Daguerre" print.; "25993" handwr. (in register book same data); "Paratype / Tyloderma /natator / Wibmer 1989" print. on yellow paper; "Tyloderma / obliquata / Hust. / Det. M.Viana [handwr.] / Museo Argentino de Ciencias Naturales [print.]" on white paper. "MACN-En / 
10217 [10218, 10219]" print. reverse.

Note: Nine parat. are at MLP (Lanteri, Suárez \& del Río 2003).

neoaffinis [Neochetina] O'Brien 1976: 163164, 167, f. 5, 11. Brasil: Mato Grosso State: Cáceres XII.1955, holot. $\hat{\jmath}$, allot. $\uparrow$ in MZSP. Argentina: Formosa, Misiones Prov. in ABC. Brasil: Mato Grosso State: Cáceres, Corumbá, Cuiabá, Salobra, Xingú; Minas Gerais State; São Paulo State: Castillo. Paraguay: Villarrica, parat. in several repositories, including MACN \& MLP.

One parat. on a card, "Rep. Argentina / A. Breyer" print., with color stripe indicative of Misiones Prov.; "Argentina / Misiones" print.; "Col. / A. Breyer" print.; "51361" handwr.; "Paratype / Neochetina / neoaffinis / C.W. O'Brien" print. on pale yellow paper. "MACN-En / 10306" print., reverse.

Note: Sixteen parat. are at MLP (Morrone \& Loiacono 1994: 18).

nigrinus [Torcus (Eutorcus)] Hustache 1939: 117-118. Buenos Aires, V.1906, from Bruch $\mathrm{n}^{\circ}$ 114.

One synt. on a card, "Rep. Argentina / Prov. Buenos Aires / 7.X.1906 / C. Bruch" print., date handwr., black frame; "Typus" handwr. by Bruch on pale green paper; "114" handwr. on pink paper; "Torcus / nigrinus / Hust," handwr. by Bruch, green frame "MACN-En / 9616" print., reverse. Ex $\mathbf{C B C}$.

nitens [Alhypera] Hustache 1926: 173-174. Chaco Prov. leg. Bruch X.1895.

breyeri [Argentinorhynchus] Brèthes 1910: Kuschel 1950b: $112 .=$ A. nitens.

One synt. on a card, "Rep. Argentina / Gob. Chaco / 1.1895 / C. Bruch" print., date handwr., black frame; "Typus" print. on pale green paper; "273" handwr. on pink paper; "Alhypera / nitens / Hust." handwr. by Bruch, red frame; "Alhypera / nitens H." handwr. by Hustache?, cut. "MACNEn / 9524" print., reverse. Ex CBC.

Note: There is another spec. from Misiones labeled "Cotypus", not mentioned in the description; see the third list, below.

nitida [Caviaphila] Hustache 1926: 177-178. Catamarca Prov: Ampajango, in nests of the rodent Cavia, from Bruch; Hualfin, in nests of the rodent Ctenomys, from Weiser; Agua Amarilla, 2650 m 28.I.1922, in nests of the rodent Ctenomys, from Weiser.
Three synt. on cards, on separate pins, "Ampajango / Catamarca" handwr. by Bruch; "ex nido de / Cavia" handwr. by Bruch; "Cotypus" print. on pale green paper, one of them also "19249" handwr. (in register book same data); "Caviaphila / nitida / Hust." handwr. by Bruch, green frame. Four synt. on cards, on two pins $(1+3$, two on the back), "Hualfin / Catam. Weiser", "ex nido de / Ctenomys" handwr. by Bruch, the pin with 1 also "Foto", "Typus" print. on pale green paper; the pin with 3 also "Cotypus" print. on pale green paper; "19249" handwr. Nine synt. on cards, on three pins $(2+3+4)$ "Agua amarilla / Catam. Weiser", "ex nido de Cteno- / mys. 2650 m. 28-I-922" handwr. by Bruch; "Typus" print. on pale green paper, the pin with 2 also "196" handwr. on pink paper; "Caviaphila / nitida / Hust." handwr. by Bruch, red frame; "Caviaphila / nitida / Hust." handwr. by Hustache?, cut. To all which lack a label with specific name, we add "Caviaphila / nitida / Hustache 1926 / Syntypus [Syntypi]" handwr. on red paper. "MACN-En / 9472 [9473 through 9487]" print., reverse. Ex CBC.

nitidulus [Cryptorhynchus] Brèthes 1910: 223224. Bolivia.

nitidulus [Eutinobothrus] Kuschel 1950b: 113. One synt. on a card, remounted, "Bolivia" handwr. on green paper, reverse white; "Repreparo / M. Viana / I-1960" handwr.; "10246" handwr.; "Cryptorhynchus / nitidulus / Brèthes" handwr. by Brèthes on whitish paper. We add "Syntypus" print. on red paper. "MACN-En / 10307" print., reverse. Ex JB.

nitidus [Amercedoidus] Hustache 1924: 93-94. Bolivia: Cuatro Ojos IX.1917 ${ }^{\lambda}$,,+ , types; Brasil: Diamantina $P$ in HUS.

nitidus [Revena]: Hustache 1938d: 142. = Amercedoides, sic.

rubiginosa [Revena] (Boheman 1836): Kuschel 1983: 42. = nitidus Hustache 1924, = sexualis Casey 1922.

One synt. pinned, "구" handwr.; "Cuatro Ojos / Bolivia" print.; "Typus" handwr. by Bruch on pale green paper; "Amercedoidus / nitidus / Hust." handwr. by Bruch, without frame; "Amercedoidus / nitidus m." handwr. by Hustache?, cut. "MACNEn / 9549" print., reverse. Ex CBC.

nothofagi [Polydrusus] Kuschel 1950c: 183184, f. 1a, photo 2. Chile: Nuble Prov.: Cordillera Chillán, type locality; Bío-Bío Prov: Antuco Vulcan; Verde Pond; Laja Pond: Los Barros; 
Trapa-Trapa; Copahue Vulcan: Termas; Malleco: Pemehue; Arauco; Contulmo, Santa Elena Park; Valdivia: Santo Domingo Stream; San José de la Mariquina; Osorno: Chanchán; Llanquihue: Frutillar; Tegualda; Aysén: Aysén Stream; Magallanes: Ultima Esperanza. Argentina: Neuquen Prov.: San Martín de los Andes; Agrio Lake. Holot., parat. in MNNC, parat. in several repositories, including MACN and MLP.

Two parat. on pointed cards, on one pin, "Paratipo" handwr. on red paper; "Chile. Pumehue / 17-1-46 Kschl. leg." handwr.; "49939" handwr.; "Polydrusus / nothofagi / Kschl. K48" handwr. on white paper, black frame. "MACNEn / 10308 [10309]“ print., reverse.

Note: One parat. is at MLP (Morrone \& Loiacono 1994: 7).

obesa [Thestonia] Hustache 1950b: 92. Corrientes Prov.: San Roque, leg. Bosq 1920, a series.

Three synt. on pointed cards, on separate pins, "San Roque / Corr. Bosq" handwr. by Bruch; "Typus" handwr. by Bruch on pale green paper. The 1st pin also "Thestonia / obesa / Hust." handwr. by Bruch, without frame; "Thestonia / obesa m." handwr. by Hustache?, cut. The 2nd \& 3rd pins also "Th. obesa" handwr. by Bruch, on a small label. "MACN-En / 9558 [9559, 9560]" print., reverse. Ex CBC.

obesus [Cylindrorrhinus] Burmeister 1879: 205. "Alto Rio Chico", near the mountain chain foot. obesus [Cylydrorhinus]: Blackwelder 1947: 812. One synt. pinned, "Cylindrorr. / obesus / Bur. Tipus [handwr.] / Museo Argentino de Ciencias Naturales [print.]" on white paper, black frame. "MACN-En / 10212" print., reverse. Ex HBC.

oblongula $[$ Linoma $]$ Hustache 1951a: 12. Buenos Aires Prov., from Bruch n 249; Quilmes 6.I.1919. [oblongulus] [Solenosternus]: Kuschel 1983: 42.

oblongulus [Solenosternus]: Wibmer \& O’Brien 1986: 296.

Two synt. on cards, on one pin, "Rep. Argentina / Prov. Buenos aires / 10.X.1906 / C. Bruch" print., date handwr., black frame; "Typus" handwr. by Bruch on pale green paper; " 249 " handwr. on pink paper; "Heterosternus / (Linoma) / oblongulus / Hust." handwr. by Bruch, without frame. We add "Linoma / oblongula / Hustache 1951 / Syntypi" handwr. on red paper. "MACN-En / 9648 [9649]” print., reverse. Ex CBC. obrieni [Pantomorus] Lanteri \& Loiácono 1990: 8. Chaco Prov.: Presidencia Sáenz Peña, 1933, Hayward, at BMNH, holot; three parat. Chaco Prov.: Resistencia, at MACN, Formosa Prov.: Fontana at MLP. Brazil: Santa Catarina State: Nova Teutonia, at MLP.

One parat. on a card, abdomen glued to another card, a plastic microvial with dissected parts; "Chaco. Argentina / Dep. Resistencia / X-XII-935J.B. Daguerre" print.; "46134" handwr. (in register book same data); "Pantomorus / obrieni / Lanteri 1990 / Paratype" print. on yellow paper. "MACN-En / 10310" print., reverse.

obrieni [Tyloderma] Wibmer 1989: 20, 91-92, f. 25, 80, 120, 141. Buenos Aires Prov., $13 \mathrm{mi} \mathrm{E}$ from Zárate 14.IV.1978 leg. O'Brien (type local.). Buenos Aires, Chaco, Corrientes, Entre Ríos, Formosa, Santa Fe, Tucumán Prov. Brasil: Rio Grande do Sul State: Pelotas. Paraguay. Uruguay. Parat. in several repositories, including MACN.

Five parat. on cards, on four pins $(1+1+1+2)$. Pins with 1: "Argentina [print.] / Delta [handwr.] / E. Ríos [print.]"; "31683" handwr. (in register book same data, and "Juan Daguerre"). Pin with 2: "Rep. Argentina / Prov. Buenos Aires / 9.VII.1905 / C. Bruch" print., date handwr., black frame; "Col. / A. Breyer" print. All: "Paratype / Tyloderma / obrieni / Wibmer 1989" print. on yellow paper. "MACN-En / 10220 [10221 through 10224]" print, reverse.

Note: Two parat. are at MLP (Lanteri, Suárez \& del Río 2003).

ogloblini [Tachygonus] Viana 1949: 78-80, 88, f. 4, pl. I: f. 4. Misiones Prov.: Loreto leg. A.A. Ogloblin, holot. ${ }^{\top}$ in MACN n ${ }^{\circ} 49305$.

Holot. on a card, “ $\bigcirc$ ” print.; "Dibujado" (depicted) handwr. with red ink; "Loreto Misiones / Rep. Argentina / Dr. A.A. Ogloblin" print.; "49305" handwr.; "Prep. $n^{\circ} 3241$ " handwr.; "Holotypus" print. on red paper; "Tachygonus / ogloblini / Viana / Holotipo $\widehat{\partial}$ [handwr.] / Museo Argentino de Ciencias Naturales [print.]" on red paper. "MACN-En 10398]" print., reverse. One slide "Antena. Holotipo ${ }^{\wedge}$ ejem. $\mathrm{N}^{\circ}$ 49305. Argentina. Misiones. Loreto. Leg. A.A. Ogloblin $\mathrm{N}^{\circ} 3241$ " handwr.; "Tachygonus ogloblini Viana. Det. M. Viana [handwr.] / Museo Arg. C. Naturales, Sección Entomología [print.]". In slide coll., under T88.

opazoi [Pentarthrum] Brèthes 1919b: 29. Chile, one spec. on "puya", leg. Opazo. castaneum 
[Pentarthrum] (Blanchard): Kuschel 1950b: 114. $=$ P. opazoi.

Holot. on a card , crushed, "Chili. 1918. R. Opazo, en 'Puya" handwr. on whitish paper; "Repreparo / M. Viana / I-1960" handwr.; "Pentarthrum / Opazoi / Brèthes" handwr. by Brèthes on whitish paper; "Pentarthrum / opazoi / Brèthes 1919 [handwr. by Kuschel] / det. G. Kuschel 1991 [print.]". We add "Holotypus" print. on red paper. "MACN-En / 10311" print., reverse. Ex JB.

Note: Kuschel, personal communication 7.XII.1990, says that $P$. opazoi Brèthes is not a synonym of $P$. castaneum (Blanchard).

orfilai [Tachygonus] Viana 1954: 162-165, f. 7, 8. Perú: Monte Tingo 6700 m, ô, o leg. Kuschel. Holot. $\partial$, allot. + , two parat. $\delta$, $q$ in GKC Two parat. $\delta$, $q$ in MACN $n^{\circ} 53014$.

Two parat. on cards, on separate pins, "개" ["ㅇ] print.; "Perú. Tingo [Tingo M'] $670 \mathrm{~m}$ 7.1947 / Weyrauch [Leg. Weyrauch]" handwr.; "53014" handwr.; "Prep. No 3048 [3049]" handwr.; "Paratypus" print. on yellowish orange paper; "Tachygonus / orfilai / Viana / Paratipo ô [o] [handwr.] / Museo Argentino de Ciencias Naturales [print.]" on yellowih orange paper. One of them also "Prep. 3174" handwr. "MACN-En / 10395 [10396]" print., reverse. Six slides, in slide coll. under T89 through T91, T94, T95, T96: Perú: Tingo María, 670 m, leg. Weyrauch: T89: "Antena. $\mathrm{N}^{\circ} 3074$ ". T90: ... N ${ }^{\circ}$ 3167, genital + , Alotipo. T91: $\mathrm{N}^{\circ} 3049$, genital + ,

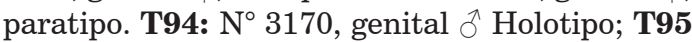
$\mathrm{N}^{\circ} 3048$ genital $\partial$ paratipo; T96 $\mathrm{N}^{\circ} 3169$ genital ô paratipo. Two slides, in slide coll. under T92, T93: Bolivia: Santa Cruz: Ichilo: Buenavista, leg. A. Martínez y Sra. II.1950, 450 mts.,T92: No 3168 genital $q$ paratipo; T93: $\mathrm{N}^{\circ} 3171$ genital $\rho$ paratipo.

pallidus [Hadromeropsis (Hadromeropsis)] Howden 1982: 67-69, f. 20, 21, 151, 166, 167, 187, map 6. Misiones Prov.: Oberá 10.V.1947, holot. in GKC. Misiones Province one $\hat{o}$ parat. in MACN. Brasil: Minas Gerais State: Vila Monte Verde, in MZSP. Paraguay: Alto Paraná: Hohenau two $\delta$ leg. Jacob 12.1939 in GKC and HAHC. Uruguay: Montevideo, in USNM. pallida [Hadromeropsis]: Wibmer \& O'Brien 1986: 69.

One parat. on a pointed card, abdomen on the same card, a plastic microvial with dissected parts, "Misiones" print.; "Pandeleteius / sp, ? nov. / je ne l'ai" handwr., cut; "Pandeleteius sp.n.?" handwr. by Bruch, red frame; "Drawing 79-38, 39" handwr.; "Paratype / Hadromeropsis [print.] / pallidus [handwr.] / A. Howden [print]" on yellow paper. "MACN-En / 9983" print., reverse. Ex CBC.

paraguayensis [Tachygonus] Viana 1954: 169-173, f. 11, 12. Paraguay: Itapuamí, leg. A. Martínez 30.XII.1950, holot. $\hat{\jmath}$, allot. + , six parat. in MACN $n^{\circ}$ 53018. Parat. in MVC (now in MLP), AMC.

Holot., allot., six parat. on cards, on separate pins, "ㄱ" ["ㅇ”] print.; "Paraguay / Itapuami / A. Martínez / 30-XI-1950 [the other 30.XII.1950]" handwr.; "35018" handwr.; "Holotypus" ["Allotypus", "Paratypus"] print. on red [red, yellow] paper; "Tachygonus / paraguayensis / Viana / Holotipo $\delta$ [Alotipo o, Paratipo ô, Paratipo o] [handwr.] / Museo Argentino de Ciencias Naturales [print.]" on red [red, red, yellow, yellow] paper. "MACN-En / 10383 (holot.) [10384 (allot.), 10385 through 10390 (parat.)]" print., reverse. Two slides "Antena. Paratipo. ejem: Paraguay, Itapuami. Leg. A. Martínez 30-X-1950" handwr.;"Tachygonus paraguayensis Viana. Det. M. Viana X-1951 [handwr.] / Museo Arg. C. Naturales. Sección Entomología [print.]". In slide collection under T97, T98.

Note: Four parat. $\delta$ are at MLP (Morrone \& Loiacono 1994: 21).

parvulus [Oxycorynus] Bruch 1916: 231-233, $4 \mathrm{f}$. Buenos Aires Prov.: Monte Hermoso leg. Carette I.1916 on Prosopanche bonacinai Spegazzini.

parvulus [Hydnorobius]: Kuschel 1959b: 268.

Four synt. on cards, on separate pins, "Mte. Hermoso / Carette leg.", (one of them also "Buenos Aires") handwr. by Bruch; "Typus" ["Cotypus", "Cotypus", "Cotypus"] print. on pale green paper; "Oxycorynus / parvulus / Bruch" handwr. by Bruch, red frame [red frame, without frame]; the "Typus" also "Hydnorobius / parvulus / (Bruch) [handwr. by Kuschel] / det. G. Kuschel / 1992 [print.]" on white paper. To the 2nd \& 3rd pins we add "Syntypus" print. on yellowish orange paper. To the 4th pin we add "Oxycorynus / parvulus / Bruch 1912 / Syntypus" handwr. on red paper. "MACN-En / 10589 [10590, 10591, 10592]" print., reverse. Ex CBC.

parvulus [Tanysphiroideus] Hustache 1926: 231. Buenos Aires leg. Bruch 3.VII.1916, the sole species in the new genus.

Two synt. on cards, on separate pins, "Rep. Argentina / Prov. Buenos Aires / 190_/ C. Bruch" 
print., black frame; "Col. / C. Bruch" print., black frame; "Typus" print. on pale green paper; one of them also "Tanysphiroideus / parvulus / Hust." handwr. by Bruch, red frame; the other one also " 306 " handwr. on pink paper; "Tanysphiroideus / n. gen. / parvulus" handwr. by Hustache?, cut. "MACN-En / 9526 [9527]" print., reverse. Ex CBC.

passiflorae [Philonis] O'Brien 1984: 236, f. 3, 7. Brasil: Bahia State: Santo Amaro Munic., Marahu, Fazenda Rocha, holot. $\rho^{\uparrow}$, allot. $\odot, 200$ parat. in several repositories, including five parat. in MACN.

Five parat. on pointed cards, on separate pins, "Brazil, Bahia / Fazenda Rocha / Marahu S/A, Santo Amaro Mun. 26-XI-1978, P. Silva" print.; "breeding in galls in vines of Passiflora sp." print.; "Paratype / Philonis / passiflorae / O'Brien 1984" print. on pale yellow paper. "MACN-En / 10338 [10339 through 10342]" print., reverse.

Note: Five $\delta$ parat.are at MLP (Morrone \& Loiacono 1994: 22).

patagonicus [Adioristus] Kuschel 1958a: 238239. Chile: Aysén: Balmaceda; Ultima Esperanza. Argentina: Chubut Prov.: Puerto Madryn; Blanco Lake; [Santa Cruz Prov.]: Viedma Lake, San Julián; Tapiloque. Holot., allot., parat. in GK; parat. in several repositories, including MACN. patagonicus [Cylydrorhinus]: Wibmer \& O'Brien 1986: 106. Nov. comb.

Two parat. on pointed cards, on one pin, "Sta. Cruz / L. Viedma / 23.2.48. Wittmer" handwr.; "Coll. / Kuschel" print. One parat. pinned, "7410" handwr.; "28." handwr.;"Patagonia / Sta. Cruz" handwr. on green paper, reverse white. Both pins: "Parátipo" print. on pale yellow paper; "Adioristus / patagonicus / n. sp. [handwr.] / Kuschel det. 1957 [print.]" on white paper. "MACN-En / 10275 [10276, 10277]" print., reverse.

Note: Six parat. $q$ are at MLP (Morrone \& Loiacono 1994: 12).

peregrinus [Pantomorus (Graphognathus)] Buchanan 1939: 14. Paper not seen.

peregrinus [Graphognathus]: Buchanan 1947: 21.

peregrinus [Naupactus]: Lanteri \& Marvaldi 1995: 222.

One parat. on a pointed card, "Gulfprt. Miss. IX,3 '37. Gladney \& Padgett” print, hardly legible; "on weeds \& grasses" print.; "37-22809 Glfprt 1” print.; “Paratype [print.] / 52355 [handwr.]" on orange paper; "52502" handwr.; "Pantomorus / (Graphognathus) / peregrinus / Buch, LB'42" handwr. on white paper. "MACNEn / 10324" print., reverse.

Notes: Two parat. $q$ are at MLP (Morrone \& Loiacono 1994: 7). Introduced into the United States of America, fide Wibmer \& O'Brien (1986: $63)$.

peruvianus [Macromerus] Brèthes 1920: 41-42. Perú: Chanchamayo.

One synt. on a card, remounted, it has a pinhole, "C.R. Vigil / Pérou" handwr. on whitish paper; "Repreparo / M. Viana / I.1960" handwr.: "Macromerus / peruvianus / Brèthes" handwr. by Brèthes on whitish paper. We add "Syntypus" print. on red paper. "MACN-En / 10323" print., reverse. $E x$ JB.

pilosipes [Rhyssomatus] Heller 1921: 35. Córdoba Prov., ${ }^{\Uparrow}$, o leg. Bruch; Mendoza Prov.: Santa Rosa, leg. Jensen-Haarup.

One synt. pinned, "Rep. Argentina / Prov. Cordoba / 21.II.1900 / C. Bruch" print., date handwr., black frame; "Typus" print. on pale green paper; "Rhyssomatus / pilosipes / Heller" handwr. by Bruch, red frame. "MACN-En / 10154" print., reverse. Ex CBC.

Note: There is another spec. labeled "Typus" from Tucumán, not mentioned in the publication, see the third list below.

pilosus [Brachystylodes] Hustache 1923: 288289. Bolivia: Cuatro Ojos XI.1917 (type); Santa Cruz IX.1917, several spec.

pilosus [Brachystylodes]: Wibmer \& O'Brien 1986: 55. = Polydacrys bolivianus Voss 1932.

One synt. on a card, "Bolivia / Santa Cruz" handwr. by Bruch; "Cotypus" handwr. by Bruch on pale green paper; "Brachystylodes / pilosus / Hust." handwr. by Bruch, without frame; "Brachystylodes / pilosus m." handwr. by Hustache?, cut. "MACN-En / 10008" print, reverse. $E x$ CBC.

pilosus [Pantomorus (Graphognathus)] Buchanan 1942: 107. Paper not seen.

pilosus [Graphognathus]: Buchanan 1947: 20

leucoloma [Graphognathus] (Boheman 1840): Warner 1975: 856. = dubius Buchanan, =imitator Buchanan, = pilosus Buchanan, = striatus Buchanan.

leucoloma [Graphognathus] (Boheman 1840): Wibmer \& O’Brien 1986: 63. = dubius Buchanan, = fecundus Buchanan, = imita- 
tor Buchanan,= pilosus Buchanan, = sriatus Buchanan. Introduced in North America.

leucoloma [Naupactus]: Lanteri \& Marvaldi 1995: 219. Nova combin.

One parat. pinned, "Repton Ala / VIII.2.39" handwr.; "USNM Paratype [print.] / 56303 pilosus [handwr.]" on red paper; "52489" handwr.; "Pantomorus / (Graphognathus) / pilosus / Buch. LB '42" handwr. on white paper. "MACN-En / 10346 " print., reverse.

platensis [Pandeleteius] Brèthes 1913a: 192. Buenos Aires City: Palermo District, leg. Zotta 2.XI.1912.

platensis [Menetypus]: Hustache 1938b: 9.

platensis [Pandeleteius]: Emden \& Emden 1939: 257.

One synt. on a card, remounted, head missing, "Bs. Aires / 2.XI.912 / A. Zotta" handwr., "Repreparo / M. Viana / I-1960"; "Syntypus" print. on red paper; "Pandeleteius / platensis / Br." handwr. by Brèthes on whitish paper. "MACN-En / 10363" print., reverse.

plumeus [Scotoeborus] Kuschel 1949: 35-37, pl. II: f. 8. Chile: Arica: Putre 3550 m; Alcérreca 4000 m; Parinacota 4400 m; Caquena 4400; Tacora: Aguas Calientes 4400 m. Perú: Coropuna 16000-17000 feet [about 5000 m]. Holot., parat. in MNS, parat. in several repositories, including MACN, MLP.

plumeus [Cylydrorhinus]: Wibmer \& O'Brien 1986: 106.

Two parat. pinned, "Parátipo" handwr. on orange paper; "Arica. Parinacota / 4400 m, 29-248 [Arica. Tacora / $4800 \mathrm{~m}, 6-3-48$ ]" handwr.; "52483" ["52482"] handwr.; "Scotoeborus / plumeus / Kschl." handwr. on white paper. One of them also "Kuschel 1949". "MACN-En / 10352 [10353]" print., reverse.

porteri (sub Porteri) [Pentarthrum] Brèthes 1913b: 39-40, f. 3. Chile: Santiago, in walnuttree wood; compares with $P$. castaneum.

castaneum [Pentarthrum] (Blanchard 1851): Kuschel 1950b: $114 .=$ P. porteri.

One synt. on a card, remounted, "Chili (Santiago) / I.1913. C.E. Porter" handwr. by Brèthes on whitish paper; "Repreparo / M. Viana / I-1960" handwr.; "Pentarthrum / Porteri Br." handwr. by Brèthes on whitish paper; "Pentarthrum / castaneum / (Blanchard, 1851) [handwr.] / det. G. Kuschel 1991 [print.]". We add "Syntypus" print. on red paper. "MACN-En / 10358" print., reverse. Ex JB. praedalta [Sibariops] Hustache 1950b: 46-47. French Guyana: Pariacabo; Guatemala.

praedata [Sibariops]: Hustache 1951b: 77. praedalta is an error, correctes to praedata.

praedatus [Sibariops]: Kuschel 1983: 43.

One synt. on a pointed card, "Guyane Française / Roches de Kourou / Coll. Le Moult" print. on green paper;"Typus" handwr by Bruch on pale green ; "Sibariops / praedata / Hust." handwr. by Bruch, without frame; "Sibariops / prædata m." handwr. by Hustache?, cut. "MACN-En / 9689" print., reverse. Ex CBC.

Note: The specific epithet was spelled praedalta by Hustache (1950), and corrected to praedata by himself (1951).

prasinus [Naupactus [(Archopactus)] cyphoides var.] Hustache 1947b: 44, 65. Chaco de Santiago del Estero Prov.: Rio Salado, from Bruch n 87.

cyphoides [Naupactus] (Heller 1921): Wibmer \& O'Brien 1986: 59. = griseomaculatus (Hustache 1923), = var. prasinus Hustache 1947, = viridimarginalis Hustache 1947.

One synt. pinned, "Rep. Argentina / Pr. Santiago d. Estero / 190_ / C. Bruch" print., black frame; "Typus" print. on pale green paper; "Archopactus / prasinus / Hust." handwr. by Bruch, red frame; "Archopactus / prasinus" handwr. by Hustache?, cut. "MACN-En / 10059" print., reverse. Ex CBC.

proximus [Atenistes] Viana 1960: 252-256, f. 2, 4, 6. Tucumán Prov. holot. $\hat{0}$, allot. + , one parat. ex CBC $n^{\circ}$ 53903. Chaco Prov.: Presidencia Roque Sáenz Peña, four parat. ex $\mathbf{C B C}^{\circ}{ }^{\circ}$ 31024. Misiones Prov.: Concepción: Santa María two parat. ex CBC n ${ }^{\circ}$ 53904. Paraguay: Puerto Casado two parat. ex $\mathbf{C B C} n^{\circ} 53905$, all in MACN. Parat. in MLP, ARR.

proximus [Toxophorus (sensu Déjean)]: O'Brien \& Wibmer 1982: 7.

proximus [Erodiscus]: O'Brien \& Wibmer 1984: 292.

Holot., allot., one parat. on cards, on separate pins, "귀"["ㅇ”, “ㄱ”] print."Rep. Argentina / Prov. Tucumán / 11.1897 [2.V.1904, 190_] / C. Bruch” print., dates handwr., black frames; "Col. / C. Bruch" print., black frame; " 53903 " handwr.; the parat. also "Atenistes / attenuatus / F." handwr. by Bruch, red frame; "Atenistes / attenuatus" handwr., cut. Four parat. on cards, on separate pins, “” [“”, “”, “ㅇ] print.; "Argentina [print.] / S. Peña [handwr.] / Chaco [print.]"; "31024" handwr. Two parat. on cards, on separate pins, “그" print.; "Misiones. Argentina / Dep. Concep. 
Sta. María / X-1947 [X-1948] M.J. Viana” print., date handwr.; "53904" handwr. Two parat. on cards, on separate pins, " $\mathrm{O}$ " print.; "Paraguay / Puerto Casado / Leg. A. Giai" handwr.; "53905" handwr. All: "Atenistes / proximus / Viana / Holotipo $\delta$ [Alotipo $\odot$, Paratipo $\delta$ ] [handwr.] / Museo Argentino de Ciencias Naturales [print.] [obverse] / M. Viana / V-1959 [handwr., reverse]" on red [red, yellowish orange] paper. "MACN-En / 10551 (holot.) [10552 (allot.), 10553 through 10561 (all parat.]" print., reverse.

Note: Four parat. $(2 \hat{\jmath}, 2$ + $)$ are at MLP (Morrone \& Loiacono 1994: 19).

pubescens [Adioristus] Hustache 1926: 186, 187-188. Catamarca Prov.: Laguna Blanca, leg. Weiser, two spec. in CBC, HUS.

pubescens [Scotoeborus]: Kuschel 1949: 31.

pubescens [Cylydrorhinus]: Wibmer \& O'Brien 1986: 106.

One synt. pinned, "Laguna Blanca / Catam. Weiser" handwr. by Bruch; "Typus" print. on pale green paper; "199" handwr. on pink paper; "Adioristus / pubescens / Hust." handwr. by Bruch, red frame; "Adioristus / pubescens m." handwr. by Hustache?, cut. "MACN-En / 9493" print., reverse. Ex CBC.

punticollis (sic) [Hyperodes] Hustache 1926: 203, 213. Buenos Aires, one spec. from Bosq. In description punticollis, in the key puncticollis. puncticollis [Listronotus]: O'Brien 1979: 267. puncticollis [Listronotus]: Wibmer \& O'Brien 1986: 119. Punticollis is a lapsus for puncticollis.

Holot. on a card, "Rep. Argentina / Prov. Buenos Aires / 190_ / C. Bruch" print., black frame; "Typus" print. on pale green paper; "229" handwr. on pink paper; "Relistrodes / puncticollis / Hust." handwr. by Bruch, red frame; "Hyperodes / puncticolli" handwr. by Hustache?, cut. "MACNEn / 9494” print., reverse. Ex CBC.

pusillus [Relistrodes] Hustache 1926. 215, 216, pl. III: f. 6. Buenos Aires Prov., from Bruch; a spec. from Bosq, somewhat larger.

pusillus [Anchodemus]: Kuschel 1950a: 14.

[pusillus] [Lixellus]: Burke 1963: 169.

[pusillus] [Listronotus]: Morrone, Marvaldi \& O'Brien 1995: 111.

Two synt. on cards, on separate pins, "Rep. Argentina / Prov. Buenos Aires /190_/ C. Bruch" print., black frame; "Typus" ["Typus" and "Foto"] print. on pale green paper; "146" handwr. on pink paper. One of them "Relistrodes / pusil- lus / Hust." handwr. by Bruch, red frame; "istrodes / pusillus Hust.", handwr. by Hustache?, cut. To the other one we add "Relistrodes / pusillus / Hustache 1926 / Syntypus" handwr. on red paper. "MACN-En / 9495 [9496]» print. reverse. $E x \mathbf{C B C}$.

rectirostris [Endalus] Hustache 1926: 218, 220-221. Buenos Aires, a couple, from Bruch.

[rectirostris] [Notiodes]: Kuschel 1971: 247.

rectirostris [Notiodes]: Wibmer \& O'Brien 1986: 190.

One synt. on a card, "Rep. Argentina / Prov. Buenos Aires / 190_ / C. Bruch" print., black frame; "Col. / C. Bruch" print., black frame; "Typus" print. on pale green paper; "147" handwr. on pink paper; "Endalus / rectirostris" handwr. by Hustache?, cut. "MACN-En / 9528" print., reverse. Ex $\mathbf{C B C}$.

reflexa [Baris] Hustache 1951a: 70-71.Tucumán Prov., from Bruch n 240.

One synt. on a card, "Rep. Argentina / Prov. Tucuman / XII.1899 / C. Bruch" print, date handwr, black frame; "Typus" handwr. by Bruch on pale green paper; "Baris / reflexa / Hust." handwr. by Bruch, green frame; "240" handwr. on pink paper. "MACN-En / 9625" print., reverse. Ex CBC.

riggii [Tachygonus] Viana 1949: 69, 70-72, 89, f. 1, pl. I: f. 1. Misiones Prov.: Dept. Concepción: Santa María, leg. Viana 1945, holot. $\odot$ in MACN sub 49302.

Holot. on a card, "O" print., "Dibujado" (depicted) handwr. with red ink; "Misiones. Argentina / Dep. Concepcion. Sta. María / 1945. M.J. Viana" print., date handwr., black frame, "49302" handwr.; "Prep. $\mathrm{N}^{\circ}$ 3000" handwr.; "Holotypus" print. on red paper; "Tachygonus / riggii / Viana / Holotipo + [handwr.] / Museo Argentino de Ciencias Naturales [print]" on red paper, black frame. "MACN-En / 10399" print., reverse. A slide "Antena $q$ holotipus ejem. $\mathrm{N}^{\circ}$ 49302. Argentina. Misiones. Dept. Concepción. Santa María. 1945. Leg. M. Viana N³000” handwr.; "Tachygonus riggii Viana. Det. M. Viana [handwr.] / Museo Arg. C. Naturales. Sección Entomología [print.]”. In slide coll. sub T99.

rospigliosii (sub Rospigliosii) [Piazurus] Brèthes 1920: 34 [sic, pro 43]-44. Perú: Chanchamayo.

varipes [Piazurus] Erichson 1847: Kuschel 1955: 312. $=$ P. rospigliosii . 
One synt. on a card, remounted, it has a pinhole, "C.R. Vigil / Pérou" handwr. on whitish paper; "Repreparo / M. Viana / I-1960" handwr.; "Piazurus / Rospigliosii / Brèthes" handwr. by Brèthes on whitish paper. We add "Syntypus" print. on red paper. "MACN-En / 10345" print., reverse. Ex JB.

rubricata [Chepagra] Hustache 1940b: 278. = Pachegra in litteris. Corrientes Prov.: San Roque, leg. Bosq 1920; Santa Fe Prov.: Fives Lille, leg. Weiser, from Bruch.

One synt. on a card, " 7 " handwr.; "San Roque / Corr. Bosq" handwr. by Bruch; "Typus" handwr. by Bruch on pale green paper; "Pachegra / rubicata (sic, pro rubricata) / Hust." handwr. by Bruch, without frame; "Pachegra / rubricata m." handwr. by Hustache?, cut. We add "Chepagra / rubricata / Hustache 1940 / Syntypus" handwr. on red paper. "MACN-En / 9617" print., reverse. Ex CBC.

rubricus [Ovanius] Hustache 1950b: 97. Brasil: Goyaz State: Jatahy. Argentina: Buenos Aires Prov.: Quilmes 8.XI.1919, leg. Bosq n 19; Martín García Island. leg. Viana.

One synt. on a pointed card, "Buenos Aires / II.1915. C.B." handwr, by Bruch; "Typus" handwr. by Bruch on pale green paper; "Ovanius / rubricus / Hust." handwr. by Bruch, without frame; "Ovanius / rubricus m." handwr. by Hustache?, cut. "MACN-En / 9550" print., reverse. Ex CBC.

rufa [Dialomia] Hustache 1939: 112. Buenos Aires Prov. 1896 from Bruch (types); $\mathrm{n}^{\circ}$ 243, XI.1899; Flores 6.XII.1918 from Bosq ${ }^{\circ} 13$; Luján 1938.

rufus [Prosaldius] Ogloblin 1930: Kuschel 1983: 42. = Dialomia rufa .

variipennis [Apinocis] Lea 1925: Zimmerman 1994: 642. = Prosaldius rufus.

Four synt. on cards, on one pin, "Rep. Argentina / Prov. Buenos Aires / 190_/ C. Bruch" print., black frame; "Typus" handwr. by Bruch on pale green paper; " 245 " handwr. on pink paper; "Prosaldius / rufus / Hust." handwr. by Bruch, without frame; "Dialomia / rufa Hust." handwr. by Bruch, green frame. "MACN-En / 9551 [9552, 9553, 9554]" print., reverse. Ex CBC.

Notes: One synt. is at MLP (Morrone \& Loiacono 1994: 27). Flores is a district in Buenos Aires City. rufipennis [Lichnus Bruchi var.] Hustache 1951b: 3. = Prolichnus Bruchi var. rufipennis in litteris. Buenos Aires Prov., from Bruch ${ }^{\circ} 122$.

bipunctatus [Lichnus] (Blanchard 1843): Wibmer \& O'Brien 1986: 340. = bruchi Hustache, = bruchi var. rufipennis.

One synt. on a card, "Rep. Argentina / Prov. Buenos Aires / 190_/ C. Bruch" print., black frame; "Typus" handwr. by Bruch on pale green paper; "Prolich. Bruchi / var. rufipennis / Hust. [handwr. by Bruch] / C. Bruch determ. [print.]". We add "Lichnus / Bruchi / var. rufipennis / Hustache 1951 / Syntypus" handwr. on red paper. "MACN-En / 10021" print., reverse. Ex CBC.

rufipes [Thanius] Hustache 1951a: 32. Neuquen Prov., from Bruch n ${ }^{\circ} 250$.

One synt. on a pointed card, "Rep. Argentina / Gob. Neuquen / 190_/ C. Bruch" print., black frame; "Typus" handwr. by Bruch on pale green paper; "250" handwr. on pink paper; "Weiske legit" handwr. by Bruch; "Thanius / rufipes / Hust." handwr. by Bruch, without frame; "Thanius / rufipes m. desi" handwr. by Hustache?, cut. "MACN-En / 9626" print., reverse. Ex CBC.

rufirostris [Baptobaris ruficollis var.] Hustache 1951a: 58. Misiones Prov. Paraguay: Hohenau.

One synt. on a card, "République Arg.ne / Haut Parana / San Ignacio. Misiones" print.; "Typus" handwr. by Bruch on pale green paper; "Baptobaris / ruficollis / Boh. / v. rufirostris Hust." handwr. by Bruch, without frame; "Baptobaris / ruficollis Boh. / v. rufirostris m." handwr. by Hustache?, cut. "MACN-En / 9627" print, reverse. $E x$ CBC.

rugosicollis [Anthobaris] Hustache 1951a: 54-55. Santiago del Estero Prov.: Rio Salado; Misiones Prov. from Bruch.

One synt. on a card, "Rep. Argentina / Gob. Misiones / X.1900 / C. Bruch" print, date handwr., black frame; "Typus" handwr. by Bruch on pale green paper; "113" handwr. on pink paper; "Anthobaris / rugosicollis / Hust." handwr. by Bruch, green frame. "MACN-En / 10022" print., reverse. Ex CBC.

ruizi (sub Ruizi) [Mimographus] Brèthes 1925: 204. Chile: Talca, leg. F. Ruiz on potatoes.

ruizi (sub Ruizi) [Macrostylus (Mimographus)]: Dalla Torre, Emden \& Emden 1936: 14.

ruizi [Pantomorus]: Buchanan 1941: 61.

ruizi [Macrostylus]: Blackwelder 1947: 793.

ruizi [Naupactus]: Kuschel 1955: 279. 
ruizi [Pantomorus]: Morrone \& Lanteri 1991: 18-22, pl. 1-4. Lectot. and paralect. designated; = Naupactus subvittatus Fairmaire \& Germain non Boheman; = N. subvittulus Emden in Dalla Torre, Emden \& Emden 1936: 23, = N. ovalipennis Hustache 1943, nomen nudum, synon. nov.; = Asynonychus variabilis; = A. variabilis var. intermedius Hustache 1947b: 139.

Lectot., one paralect. on cards, on separate pins, "Prov. Talca / 1923 / F. Ruiz" handwr.; "Repreparo / M. Viana / I-1960" handwr.; "ataca las papas" handwr.; "Mimographus / Ruizi Brèthes" handwr. by Brèthes; "Mimographus ruizi Brèthes / LECTOTIPO [PARALECTOTIPO] Des. por Morrone / \& Lanteri 1988" print. on red paper; the lectot., also "Lectotype" print. with red ink on white paper. "MACN-En / 10343 (lectot.) [10344 (paralect.)]” print., reverse. Ex JB.

rustica [Baris] Hustache 1951a: 71-72. Buenos Aires Prov., from Bruch $\mathrm{n}^{\circ}$ 237; Delta of the Paraná River: Chaná Stream, leg. Bosq.

One synt. on a card, "Rep. Argentina / Prov. Buenos Aires / 12.XII.1912 / C. Bruch" print., date handwr., black frame; "Typus" handwr. by Bruch on pale green paper; " 237 " handwr. on pink paper; "Baris / rustica / Hust." handwr. by Bruch, green frame. "MACN-En / 9628" print., reverse. Ex CBC.

sastrei [Entimus] Viana 1957: 6-10, f. C, D, pl. I: f. 2. Buenos Aires Prov: Punta Lara, leg. Bruch I.1928, holot. $\sigma^{\top}$, allot. $+\mathrm{n}^{\circ} 53322$, parat. four $\widehat{\gamma}$, five $\odot \mathrm{n}^{\circ} 53323$; Buenos Aires "col. antigua" $\mathrm{n}^{\circ} 53318$, parat. two ${ }^{\lambda}$, one $\circ$; Buenos Aires Prov., leg. Bruch $\mathrm{n}^{\circ}$ 53324, parat. three $\odot$; Buenos Aires ex coll. Breyer $\mathrm{n}^{\circ} 53325$ parat. one $\delta$, two $\circ$; Entre Ríos Prov.: Paranacito, leg. Förster XI.1953 $n^{\circ} 53326$, parat. five ${ }^{\lambda}$, three +; Martínez Stream, leg. Walz XI.1953 n 53328, parat. six $\hat{\partial}$, four $q$, all in MACN. Other parat. in several repositories.

sastrei [Entimus]: Wibmer \& O'Brien 1986: 100. $=$ Entimus nobilis auctorum non Olivier 1790 .

Holot., allot., nine parat. pinned, the holot. with genit. on a card, "Dibujado" handwr. (depicted); “ ㅊ", [“”"] print.; "Punta Lara / 29-I-928. C.B." handwr. by Bruch, "Col. / C. Bruch" print., black frame; "53322" ["53323"] handwr. Three parat. pinned, "O” print.; "Rep. Argentina / Prov. Buenos Aires / 2.[month illegible].1897 / C. Bruch" print., date handwr., black frame; "53324" handwr. Eight parat. pinned, "그" ["O"] print.; "Entre Ríos / Paranacito / 20-I1953" handwr.; "53326" handwr. Three parat. pinned, “ㅈ” [“”] print.; "Col. / Antigua" print. on whitish paper; " 53318 ", one of them also "Buen. / Ayres" print. on green paper; "nobilis Oliv. / Am. merid." handwr. by Burmeister, black frame, originally fastened to the bottom of the drawer. Ten parat. pinned, “그 [“ㅇ"] print.; "Entre Ríos / Rio Martínez / Leg. F. Walz" handwr.; "53328" handwr. Three parat. pinned, “ㄱ" [“”] print.; "Argentina / A. Breyer" print., a color stripe indicative of Buenos Aires. All: "Holotypus" ["Allotypus", "Paratypus"] print. on red [red, yellow] paper; "Entimus / sastrei / Viana / Holotipo ô [Alotipo o, Paratipo ô, Paratipo ?]" handwr. on red [red, yellow, yellow] paper. "MACN-En / 10435 (holot.) [10436 (allot.), 10437 through 10460 parat), 10509 through 10520 (parat.)]" print., reverse.

Note: Eleven $\delta$ and fourteen $\$$ parat. are at MLP (Morrone \& Loiacono 1994: 12-13).

scrobiculatus [Cylindrorrhinus angulatus var.] Burmeister 1879: 203. [Santa Cruz Prov.]: Rio Chico.

scrobiculatus [Cylydrorhinus angulatus v.]: Blackwelder 1947: 812.

angulatus [Cylydrorhinus] Guérin 1841: Kuschel 1950a: 12. = scrobiculatus.

angulatus [Cylydrorhinus]: Wibmer \& O'Brien 1986: 103. = Adioristus rubripes Philippi 1862 fide Kuschel 1950, = A. rugatus Philippi 1862 fide Kuschel 1950, = scrobiculatus fide Kuschel 1950.

Three synt. (all three?) pinned, one of them: "angulatus con una ind. "var." / sera scrobiculatus Burm. [handwr.] / Museo Argentino de Ciencias Naturales [print.]" on white paper; "Cylindrorh. / angulatus / rugatus (Philippi) [handwr.] / Kuschel det. 1954 [print.]" on white paper. We add to all three "Cylindrorrhinus / angulatus / var. scrobiculatus / Burmeister 1879 / Syntypus" handwr. on red paper. "MACN-En / 10195 [10196, 10197]” print., reverse. Ex HBC.

seminebulosus [Conotrachelus] Brèthes 1910: 219-220. Bolivia.

histrio [Conotrachelus] Boheman 1859: Hustache 1926: 254. = C. seminebulosus.

coelebs [Conotrachelus] Boheman 1837: Wibmer \& O'Brien 1986: 157. = C. histrio nov. synon.

One synt.? on a card, remounted, "Typus" handwr. on green paper; "Repreparo / M. Viana / I-1960" handwr.; "Typus" print. on red paper; "Conotrachelus / seminebulosus Brethes" handwr. on orange paper. "MACN-En / 10361" print., reverse. Ex JB. 
Notes: The spec. has no locality label; it was not labeled by Brèthes, setting some doubt on its type condition. There is another spec. from Misiones Prov., not mentioned in the description, see the third list, below.

semirufus [Hiotus] Hustache 1950a: 37. Misiones Prov: Paraná River. Paraguay: Hohenau.

One synt. on a pointed card, "Hohenau / Paraguay" print.; "Typus" handwr. by Bruch on pale green paper; "Hiotus / semirufus / Hust." handwr. by Bruch, without frame; "Hiotus / semirufus m. 285" handwr. by Hustache?, cut. “MACN-En / 9555” print., reverse. Ex CBC.

semirufus [Parallelosomus] Hustache 1940b: 297 (sic, pro 279). In a key; Santa Fe Prov., type, from Bruch $n^{\circ}$ 293; Rafaela 8, 22.XI.1932, leg. Denier; Fives Lille, leg. Weiser; Buenos Aires Prov., leg. Bosq; Córdoba Prov., leg. Bruch.

semirufus [Lipancylus]: Kuschel 1983: 39.

Two synt. on cards, on one pin, "Rep. Argentina / Prov. Santa Fe / 190_ / C. Bruch" print., black frame; "Typus" handwr. by Bruch on pale green paper; "292" handwr. on pink paper; "Parallelosomus / semirufus / Hust." handwr. by Bruch, green frame. One synt. on a card, "Fives Lille / S. Fé. Weiser" print.; "Fototypus" handwr. by Bruch on pale green paper; we add "Parallelosomus / semirufus / Hustache 1940 / Syntypus" handwr. on red paper. Four synt. on cards, on two pins (2+2), "Fives Lille / S. Fé. Weiser" print.; "Cotypus" handwr. by Bruch on pale green paper; "Parallelosomus / semirufus / Hust. [handwr. by Bruch] / C. Bruch determ. [print.]" on white paper. "MACN-En / 10043 [10044 through 10049]" print., reverse. Ex CBC.

Note: Four synt. are at MLP (Morrone \& Loiacono 1994: 27).

seriatosetulosa [Methyorrhina] Hustache 1951a: 61. Tucumán Prov. from Bruch nº 268.

One synt. on a card, "Rep. Argentina / Prov. Tucuman / 5.III.1900 / C. Bruch" print., date handwr., black frame; "Typus" handwr. by Bruch on pale green paper; "268" handwr. on pink paper; "Methyorrhina / seriatosetulosa / Hust." handwr. by Bruch, green frame. "MACN-En / 9629" print., reverse. Ex CBC.

sharpi [Pseudopantomorus] Heller 1921: 22-23, f. 1. Tucumán Prov., from Bruch. Paraguay, in coll. Faust.
Sharpi [Parapantomorus]: Dalla Torre, Emden \& Emden 1936: 25.

fluctuosus [Parapantomorus] (Boheman 1840): Kuschel 1955: 280. = P. sharpi. Nova combin.

One synt. on a card, "Rep. Argentina / Prov. Tucuman/16.I.1904/C. Bruch" print., date handwr., black frame; "K.M. Heller" print.; "Typus" print. on pale green paper; "Pseudopantomorus / Sharpi / Heller" handwr. by Bruch, red frame. "MACN-En / 10072" Print., reverse. Ex CBC.

Note: There are two further spec. from Misiones Prov., labeled "Typus", not mentioned in the publication, see the third list, below.

similis [Macromerus] Brèthes 1920: 42. Perú: Chanchamayo.

numenius [Macromerus] Erichson 1847: Kuschel 1950b: $114 .=$ M. similis.

One synt. on a card, remounted, it has a pinhole, "C.R. Vigil / Pérou" handwr. on whitish paper; "Repreparo / M. Viana / I-1960" handwr.; "Macromerus / similis Brèthes" handwr. by Brèthes on whitish paper. We add "Syntypus" print. on red paper. "MACN-En / 10322" print., reverse. Ex JB.

simplex [Cratosomus] Brèthes 1920: 33-34. Perú: Chanchamayo.

One synt. on a card, remounted, it has a pinhole, "C.R. Vigil / Pérou" handwr. on whitish paper; "Repreparo / M. Viana / I-1960" handwr.; "Cratosomus / simplex Brèthes"

handwr. by Brèthes on whitish paper. We add "Syntypus" print. on red paper."MACN-En / 10321” print., reverse. Ex JB.

singularis [Cyrtobagous] Hustache 1929: 228. Brasil: Mato Grosso [do Sul] State: Corumbá, from Staudinger; a long series, types in HUS.

Two synt. on cards, on separate pins, "Corumba / Matt. Grosso" print.; "Col. / C. Bruch" print., black frame; "Typus" handwr. by Bruch on pale green paper. One of them also "Cyrtobagous / singularis m." handwr. by Hustache? reverse black. The other one also "Cyrtobagous / singularis / Hust." handwr. by Bruch, without frame. "MACN-En / 9529 [9530]" print. reverse. Ex CBC.

sordidus [Cylindrorrhinus] Burmeister 1879: 206. "Alto Río Chico", near the foot of the mountain chain.

sordidus [Cylydrorhinus]: Blackwelder 1947: 812.

sordidus [Paulsenius]: Kuschel 1950a: 12-13. 
= Listroderes schythei Germain 1895.

sordidus [Cylydrorhinus]: Wibmer \& O'Brien 1986: $106 .=$ L. schythei. In Cylydrorhinus fide Kuschel in litteris.

One synt. pinned, "Cylindror. / sordidus / Burm. Tipo [handwr.] / Museo Argentino de Ciencias Naturales [print.]" on white paper. We add "Cylindrorrhinus / sordidus / Burmeister 1879 / Syntypus" handwr. on red paper. "MACN-En / 9604" print., reverse. Ex HBC.

sparsepunctata [Tripusidia] Hustache 1951b: 64. French Guyana: Nouveau Chantier; Gourdonville, Cayenne, "une série de spécimens".

One synt. on a card, "Guyane Franç.se / Nouveau Chantier / Collection Le Moult" print.; "Tripusidia / sparsepunctata / Hust." handwr. by Bruch, without frame "Tripusidia / sparsepunctata m." handwr. by Hustache?, cut. One synt. on a card, "Guyane Française / Gourdonville / Rivière de Kourou" print. Both: "Typus" handwr. by Bruch on pale green paper. We add "Tripusidia / sparsepunctata / Hustache 1951 / Syntypus" handwr. on red paper. "MACN-En / 9614 [9615]" print., reverse. Ex CBC.

speculifer [Chalcodermus] Heller 1906: 30-31. Tucumán Prov.; Paraguay, from Bruch. In SMTD under 18404.

Two synt. on cards, on separate pins, "Rep. Argentina / Prov. Tucuman / 190_ [XII.1899] / C. Bruch" print., date handwr., black frame; "Typus" print. on pale green paper. One of them also "K.M. Heller" print.; "Chalcodermus / speculifer / Heller. tipo" handwr. by Bruch, red frame. To the other one we add "Chalcodermus / speculifer / Heller 1906 / Syntypus" handwr. on red paper. "MACN-En / 10151 [10152]" print., reverse. Ex CBC.

spegazzinii (sub Spegazzinii) [Otidocephalus] Brèthes 1910: 14-15. Jujuy Prov., one spec. leg. Spegazzini.

spegazzinii [Myrmex]: Blackwelder 1947: 835. spegazzinii $[$ Myrmex]: Wibmer \& O'Brien 1986: 199.

Holot. on a card, remounted, "Jujuy" handwr. on green paper, reverse white; "Repreparo / M. Viana / I-1960" handwr.; "7274" handwr.; "Holotypus" print. on red paper; "Otidocephalus / Spegazzinii Brèthes" handwr. by Brèthes on whitish paper. "MACN-En / 10320" print., reverse. Ex JB.

sphaeralceae [Paraceratopus $]$ Brèthes
1910: 217-218. Frequent the year around on Sphaeralcea bonariensis (Malvaceae); several spec. in MACN.

australis [Macrorhoptrus]: Wibmer \& O'Brien 1986; 202. Nom. nov. pro sphaeralceae Brèthes 1910, non sphaeralciae sic, lapsus, Pierce 1908.

Five synt. on cards, remounted on two pins $(1+4)$, "Bs. Aires / 16.IV.'10 [19.IV.'10] J.B." handwr., black frame; "Repreparo / M. Viana / I-1960" handwr.; "Paraceratopus / sphaeralceae Brèthes" handwr. by Brèthes on whitish paper. The 1st pin also " 10415 " handwr. To both pins we add "Syntypus" ["Syntypi"] handwr. on red paper. "MACN-En / 10315 [10316 through 10319]" print., reverse. $E x$ JB.

stegotopsides [Garnia] Hustache 1950b: 93. Tucumán Prov. 1897, from Bruch n 288.

One synt. on a card, "San Pedro Colalao / Tucum. Weiser" handwr. by Bruch; "Typus" handwr. by Bruch on pale green paper; "288" handwr. on pink paper; "Garnia / stegotopsides / Hust." handwr. by Bruch, green frame. "MACNEn / 9556" print., reverse. Ex CBC.

sternicornis [Derelomus] Bondar 1941a: 277280, f. 2, 3. Brasil: Bahia State: Munic. Nazaré, fazenda Copiobá, leg. Bondar 20.XI.1940, hundreds of spec. on $\delta$ flowers of Diplothemium caudescens (Palmae), type and parat. in BON. sternicornis [Diplothemiobius]: Kuschel 1952b: 272. Type species of the new genus.

sternicornis [Derelomus]: Vaurie 1953: 35. Designates lectot. ex 29 'cot.'; twenty-one 'cot.' in USNM, fide Warner in litt.

One paralect. on a pointed card, "fazenda Copioba, municip. Nazaré", "Estado da Bahia XI:20:40. Brazil. Bondar leg.", "flores Diplothemium caudescens" print.; "53520" handwr.; "Cotype / Derelomus / sternicornis / Bond." print. on red paper. "MACN-En / 10360" print., reverse.

Notes: Vaurie (1953: 35) stated that even though a type and a parat. have been mentioned, all the spec. were labeled as 'cotype'. She designated a $\delta$ lectot. ex 29 'cot.'; twenty-one 'cot.' are at USNM, Washington, USA. fide Warner in litt.

striata [Ctenomyophila] Hustache 1926: 179, 184-185, pl. I: f. 9. Chaco de Santiago del Estero Prov., leg. Wagner; Córdoba Prov.: Alta Gracia, leg. Bruch.

[striatus] [Hormops]: Kuschel 1964: 423.

Six synt. on five pins $(1+1+1+1+2)$, "Alta Gracia. La Granja / Sierras de Córdoba / 1.I.924 
[ ---, 10.I.925, 9.I.925, 20.II.925] / C. Bruch leg." print., dates handwr.; "Typus" [on 1st, 3rd, 5th], [ "Cotypus" [on 2nd, 4th]. The 1st pin also "Ctenomyophila / striata / Hust." handwr. by Bruch, red frame; "nomyophila / striata m." handwr. by Hustache?, cut. The 2nd pin also "19247" handwr. (in register book same data); "Ctenomyophila / striata / Hust." handwr. by Bruch, green frame. The 3rd pin also "Foto" print. on pale green paper. The 4th pin also "19247" handwr. (in register book same data). To the 3rd, 4th \& 5th pins we add "Ctenomyophila / striata / Hustache 1926 / Syntypus [Syntypi]" handwr. on red paper. "MACN-En / 9497 [9498 through 9502]" print., reverse. Ex CBC.

striatipennis [Anopsilus] Bondar 1942b: 255256. = Anopsilus striatipennis Hustache in litteris. Brasil: Bahia State, on Canna denudata [Cannaceae], 'cotypi' in BON, FMNH.

striatipennis [Anopsilus]: Hustache 1951a: 65-66. Misiones Prov.; Buenos Aires leg. Bosq; Martín García Island leg. Viana. Paraguay: Hohenau. Brasil: Bahia State.

striatipennis [Anopsilus]: Vaurie 1953: 35-36. Designates lectot., ex two 'cot.' Other 'cot.' in FMNH.

striatipennis [Anopsilus] Bondar 1942b: Wibmer \& O'Brien 1986: 305. = Anopsilus striatipennis Hustache 1951.

One paralect. on a card, "Rep. Argentina / Prov. Buenos Aires / 12.1894 / C. Bruch" print., date handwr., black frame; " 120 " handwr. on pink paper; "Anopsilus / striatipennis / Hust." handwr. by Bruch, without frame; "Anopsilus / striatipennis m." handwr. by Hustache?, cut. One paralect. on a card, "Bs. Aires / Palermo / 9.8.914" handwr. by Bruch. One paralect. on a card, "République Arg.ne / Rio Parana / Territoire des Missions" print. All three: "Typus" handwr. by Bruch on pale green paper. To the 2nd \& 3rd ones we add "Anopsilus / striatipennis / Hustache 1951 / Syntypus" handwr. on red paper. "MACN-En / 10032 [10033, 10034]" print., reverse. Ex CBC.

Note: Bondar (1942b: 255-256) says that A. striatipennis Hustache 1940 is a name in litteris [it was not published until 1951].

striatus [Pantomorus (Graphognathus)] Buchanan 1942: 108. U.S.A:: Louisiana State: New Orleans, 5-VII-1930, holot. $q$ at USNM; several parat. from New Orleans and vicinities. leucoloma striatus [Graphognathus]: Buchanan 1947: 20.

leucoloma [Graphognathus] (Boheman 1840):
Warner 1975: 856. = dubius $($ Buchanan $),=i m i-$ tator $($ Buchanan $),=$ pilosus $($ Buchanan $),=$ striatus (Buchanan).

leucoloma [Graphognathus]: Wibmer \& O'Brien 1986: 63. = dubius (Buchanan), = fecundus (Buchanan); = imitator (Buchanan), = pilosus (Buchanan), = striatus (Buchanan).

leucoloma [Naupactus]: Lanteri \& Marvaldi 1995: 219.

One parat. pinned "N. Orleans. Area VI to VIII'39" handwr.; "Paratype striatus 56307 USNM" print. and handwr. on red paper; "52487" handwr. "MACN-En / 10314" print., reverse.

Notes: There is another spec. identified as "Pantomorus (Graphognathus) striatus Buch. LB'42" handwr. on white paper, but not labeled as a type. Two parat. are at MLP (Morrone \& Loiacono 1994: 8). Introduced into U.S.A. from South America.

subfasciata [Myelantella] Hustache 1940b: 280. = Sibariella in litteris. Brasil: Goiaz State: Jatahy ex Donckier, a series.

One synt. on a card, "Jatahy / Goyaz" print.; "Typus" handwr. by Bruch on pale green paper; "Sibariella / subfasciata / Hust." handwr. by Bruch without frame; "Sibariella / subfasciata m." handwr, by Hustache?, cut. We add "Myelantiella / subfasciata / Hustache 1940 / Syntypus" handwr. on red paper. "MACN-En / 9605" print., reverse. Ex CBC.

sulfureovittatus (sub sulfureo-vittatus) [Enoplopactus] Heller 1921: 26, f. 5. Santiago del Estero Prov., $\widehat{O}$, o leg. Bruch.

One synt. pinned, "Rep. Argentina / Pr. Santiago d. Estero / 190_ / C. Bruch" print., black frame; "Typus" print. on pale green paper; "Enoplopactus / sulfureo-vittatus / Heller" handwr. by Bruch, red frame; "Enoplopactus / sulfureo-vitt" handwr. by Heller? on white paper, cut. "MACN-En / 10058" print., reverse. Ex CBC.

superbus [Hadromerus] Heller 1921: 29-30. Tucumán and Córdoba Prov., leg. Bruch. superbus [Hadromeropsis]: Günther \& Zumpt 1933: 103.

superbus [Hadromeropsis]: Howden 1982: 49. Designates as lectot. a male labeled "Rep. Argentina, Prov. Tucumán, II-1906, C. Bruch, K.M.Heller"

superba [Hadromeropsis]: Wibmer \& O'Brien 1986: 69.

Lectot., two paralect. pinned, "Rep. Argentina 
/ Prov. Tucuman / II.1906 / C. Bruch" print., date handwr., black frame; "Typus" ["Cotypus"] print. [handwr. by Bruch] on pale green paper; "Hadromerus / superbus / Heller i. l." handwr. by Bruch, red [green] frame; "Hadromerus / superbus / Heller m. i. l." handwr. by Heller?, cut; "Lectotype [print.] / Hadromerus / superbus / Heller [handwr.] / des. A. Howden [print.]" on red paper, one of the paralect. also " 19245 " handwr. (in register book same data), but not labeled by Heller nor by Howden. One paralect.? pinned, "Rep. Argentina / Prov. Catamarca / 9.III.1907 / C. Bruch" print., date handwr., black frame; "Typus" print. on pale green paper; "Hadromerus / superbus / Heller" handwr. by Bruch, red frame; "Paralectotype [print.] / Hadromerus / superbus / Heller [handwr.] / des. A. Howden [print.]" on yellow paper. "MACN-En / 10186 (lectot.) [10187, 10188, 10189 (all paralect.)]" print., reverse. Ex CBC.

Note: The spec. from Catamarca was not mentioned in the original publication, but it was labeled "Paralectotype" by Howden. Its type condition is obscure.

tarapacanus [Strangaliodes (Chersostrangalio des)] Kuschel 1949: 25-26, f. 3 c, d, pl. II: f. 2. Chile: Pisagua: Champaja $2900 \mathrm{~m}$, on Opuntia ficus-indica (Cactaceae), holot. in MNNC, allot. in GK, parat. in several repositories, including MACN.

azurescens [Strangaliodes] Kuschel 1949: Wibmer \& O’Brien 1986: 87. = S. tarapacanus Kuschel.

One parat. on a pointed card, "Parátipo" handwr. on red paper; "Pisagua / Champaja $2900 \mathrm{~m}$ 18-2-48", "CHILE" handwr.; "49935" handwr.; "Strangaliodes / (Chersostrang.) / tarapacana / Kschl. / K 48" handwr. on white paper, black frame. "MACN-En / 10351" print. reverse.

testaceus [Azygides (Neoazygides)] Hustache 1940a: 142. Buenos Aires Prov. IV.1897, leg. Bruch; Tigre 1938 leg. Viana.

One synt. on a card, "Rep. Argentina / Prov. Buenos Aires / 190 / C. Bruch" print., black frame; "Typus" handwr. by Bruch on pale green paper; "127" handwr. on pink paper; "Neoazygides / testaceus / Hust." handwr. by Bruch, without frame; "Neoazygides / testaceus m." handwr. by Hustache?, cut. We add "Azygides / (Neoazygides) / testaceus / Hustache 1940 / Syntypus" handwr. on red paper. "MACNEn / 10027" print., reverse. Ex CBC.

Note: Tigre refers to the distal (lower) portion of the delta of the Paraná River. thaliae [Amalactus] Viana 1951: 32, 36-39, 45, f. 4, 5, pl. II. Buenos Aires Prov.: Tigre, on Thalia

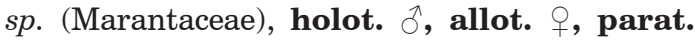
10 , 10 in MACN $n^{\circ} 51564$ through 51567, parat. in MVC [now in MLP].

thaliae [Amalactus nigritus subsp.]: Kuschel 1959a: 509.

Holot., allot., 20 parat. on cards, "구 [“ㅇ] print.; "Buenos Aires / Argentina [print.] / Tigre 1948 [handwr.] / M.J. Viana [print.]"; "s/ Talia sp." handwr.; "51564" ["51565", "51566", "51567"] handwr.; "Holotypus" ["Allotypus", "Paratypus"] print. on red [red, yellow] paper; "Amalactus / thaliae / Viana / Holotipo ô [Alotipo

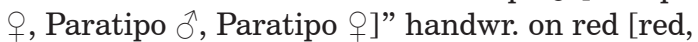
yellow, yellow] paper. "MACN-En / 10413 (holot.) [10414 (allot.), 10415 through 10434 (all parat.)]" print., reverse.

Note: Tigre refers to the distal (lower) portion of the delta of the Paraná River.

titara [Dahlgrenia] Bondar 1942a: 36-38. Brasil: Espirito Santo State: municipio São Mateus, Braço do Norte Stream VI.1941, Bondar leg. 410 spec., reared from fruits of "titara" (Desmoncus polyacanthos, Palmae), "cotypes" in BON and several other repositories, MACN not mentioned.

titara [Stethobaropsis]: Kuschel 1983: 42.

titara [Dahlgrenia]: Vaurie 1953: 37. Designates lectot. ex 173 'cot.'; other 'cot.' in FMNH, IBSP, MZSP.

Two paralect. on pointed cards, on separate pins, "Braço do Norte", "mun. S. Mateus", "Estado do Espirito Santo, VI: - :41. Brazil. G. Bondar leg.", "fruits of Desmoncus polyacanthus" print. on white paper, "53518" handwr.; "Cotype / Dahlgrenia / titara / Bond." print. on red paper. "MACN-En / 10356 [10357]" print., reverse

\section{tornowii (sub Tornowii) [Sphenophorus]} Brèthes 1910: 226. Tucumán Prov.

tornowii (sub Tornowii) [Rhodobaenus]: Bruch 1915: 426.

tornowii (sub Tornowii) [Calendra]: Csiki 1936: 62

tornowii [Homalostylus]: Kuschel 1950a: 20.

tornowii [Rhodobaenus]: Wibmer \& O'Brien 1986: 376.

One synt. on a card, remounted, it has a pinhole, "Tucumán" handwr. on green paper, reverse white; "Repreparo / M. Viana / I-1960" handwr.; "Sphenophorus / Tornowii Brèthes" handwr. by Brèthes on whitish paper. One synt. on a card, remounted, it has a pinhole, "Rep. 
Argentina / A. Breyer" print., with a color stripe indicative of Tucumán; "Repreparo / M. Viana / I-1960" handwr.; "Sphenophorus / tornowii Brèth." handwr. on white paper. We add to both "Syntypus" handwr. on red paper. "MACN-En / 10349 [10350]" print., reverse. Ex JB.

torresi [Bondariella] Bondar 1942a: 22-24. Brasil: Bahia State: munic. Santa Tereza, Santa Luzia. 'Cotypes' in BON and other repositories, MACN not mentioned. About 300 spec. in 0 flowers of Cocos vagans (Palmae).

torresi [Bondariella]: Vaurie 1953: 37. Designates lectot. ex 248 'cot.'; other 'cot.' in FMNH, IBSP.

One paralect. on a card, "municipio S. Tereza or S. Luzia" print.; "Estado da Bahia / Brazil G. Bondar leg." print.; "on flowers / Cocos vagans" print. ; "53514" handwr.; "Cotype CNHM / Bondariella / torresi / Bondar" print. on red paper. "MACN-En / 10280" print., reverse.

Note: Eleven paralect. (sub parat.) are at MLP (Morrone \& Loiacono 1994: 28).

triangularis [Megavalius (sic, pro Megavallius)] Hustache 1950b: 107. French Guyana: Nouveau Chantier; Saint Laurent du Maroni, Roches de Kourou.

One synt. on a card, "Juillet" print.; "Typus" handwr. by Bruch on pale green paper; "Megavallius / triangularis / Hust." handwr. by Bruch, without frame; "Megavallius / triangularis m." handwr. by Hustache?, cut. "MACN-En / 9557" print., reverse. Ex CBC.

tucumanensis [Auletobius] Hustache 1926: 232, pl. V: f. 3. Tucumán Prov., from Bruch.

Three synt., one of them without head, on cards, on two pins $(1+2)$, "Rep. Argentina / Prov. Tucuman / 190_ / C. Bruch" print., black frame; "Typus", "Foto" ["Typus"] print. on pale green paper; The 1st pin also "Auletobius / tucumanensis / Hust." handwr. by Bruch, red frame; "Rhynchites / tucumanensis n. sp." handwr. by Hustache?, cut. To the 2nd pin we add "Auletobius / tucumanensis / Hustache 1926 / Syntypus" handwr. on red paper. "MACN-En / 10190 [10191, 10192]" print., reverse, Ex CBC.

tucumanus [Rhigopsidius] Heller 1906: 8-9, pl. I: 3, 3 a, b. Tucumán Prov. from Bruch V.1903, in SMTD n ${ }^{\circ} 17995$.

tucumanus [Rhigopsidius]: Emden 1952: 744. $=R$. piercei Heller 1936 .

tucumanus [Rhigopsidius]: Wibmer \& O’Brien 1989b: 30. Valid name, not a synonym of piercei (Morrone \& Loiácono 1992).

Two synt. pinned, "Rep. Argentina / Prov. Tucuman / V.1903 / C. Bruch" print., date handwr., black frame; "Typus" print. on pale green paper. One of them also "Rhigopsidius / tucumanus / Heller. Tipo" handwr. by Bruch, red frame "K.M. Heller" print. To the other one we add "Rhigopsidius / tucumanus / Heller 1906 / Syntypus?" handwr. on red paper. "MACN-En / 9701 [9702]" print., reverse. Ex CBC.

unicolor [Adioristus villosulus var.] Hustache 1926: 189. Catamarca Prov.: Laguna Blanca, leg. Weiser. Collected together with the typical form. [unicolor] [Scotoeborus villosulus var.]: Kuschel 1949: 34-35, pl. II: f. 7. = Adioristus griseus Germain 1911 nom. nud.. Includes locus typicus of unicolor.

villosulus [Cylydrorhinus]: Wibmer \& O'Brien 1986: 107. = griseus Germain nom. nud., = unicolor Hustache.

Two synt. on cards on one pin, "Laguna Blanca / Catam. Weiser" handwr. by Bruch; "Typus" print. on pale green paper; "197" handwr. on pink paper; "A. villosulus / Hust. / var. unicolor / Hust." handwr. by Bruch, red frame; "illosulus / unicolor m" handwr. by Hustache?, cut. "MACNEn / 9503 [9504]" print., reverse. Ex CBC.

unicolor [Geraeus] Hustache 1924: 97-98. Bolivia: Santa Cruz IX.1917, types; a series; Villa Montes XI.1917, one spec.

unicolor [Thestonia]: Kuschel 1983: 42.

One synt. on a pointed card, "Santa Cruz / Bolivia" print.; "Typus" handwr. by Bruch on pale green paper; "Thestonia / unicolor / Hust." handwr. by Bruch, without frame; "Thestonia / unicolor m." handwr. by Hustache?, cut. We add "Geraeus / unicolor / Hustache 1924 / Syntypus" handwr. on red paper. "MACN-En / 10563" print., reverse. Ex CBC.

urvilleae [Achia] Clark, Burke \& McKay 2007: 545. Brasil: Santa Catarina State: Nova

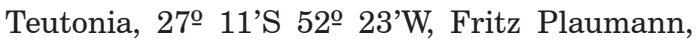
VIII-1954, 300-500m, holot. $\delta$ at MZSP. thirteen parat. Brasil: Nova Teutonia.Two parat $\hat{\jmath}$, + Argentina, Misiones Prov., one at MACN.

One parat. on a pointed card, "Argentina. Misiones Province / R N ${ }^{\circ}$ 17, 3 km E Pozo Azul / 19 Aug. 2006 / McKay col." print.; "Emerged from fruits of / Urvillea ulmacea [print.] G \&" handwr., "Paratype / Achia / urvilleae / Clark and Burke" print. on sky-blue paper, remainings of a black frame; "MACN-En / 6853" print. reverse. 
vallium [Smicronyx] Kuschel 1949: 52-54, pl. III: f. 9. Chile: Arica: Camarones Valley: Huancarane, on Cuscuta; Pisagua: Miñita 2400 m; Iquique: Tarapacá 1400 m. Holot., one parat. in MNNC, parat. in several repositories, including two parat. in MACN.

Twoparat. on pointed cardsononepin, "Parátipo" handwr. on red paper; "Arica: Huancarane 20-1146" handwr.; "CHILE" handwr.; "49940" handwr.; "Smicronyx / vallium / Kschl / K 48" handwr. on white paper, black frame. "MACN-En / 10335 [10336]" print., reverse.

Note: Two parat. are at MLP (Morrone \& Loiacono 1994: 19).

variabilis [Bolivianus] Hustache 1923: 293. Bolivia: Villa Montes (types); Santa Cruz: Cuatro Ojos; Yacuiva, Lagunillas, several spec.

variabilis [Eustales (Bolivianus)]: Voss 1954: 200.

variabilis [Bolivianus]: Wibmer \& O'Brien 1986: 84.

One synt. on a card, "Bolivia / Santa Cruz" handwr. by Bruch; "Cotypus" handwr. by Bruch on pale green paper; "Bolivianus / variabilis / Hust." handwr. by Bruch, red frame; "Bolivianus / variabilis m." handwr. by Hustache?, cut. "MACN-En / 10009" print., reverse. Ex CBC.

variabilis [Endalus] Hustache 1926: 219. Buenos Aires City: Palermo District, from Bosq; Santa Fe Prov.: Fives Lille, from Weiser \& from Bruch.

[variabilis] [Notiodes]: Kuschel 1971: 247.

nanus [Notiodes] Boheman 1859: Wibmer \& O'Brien 1986: 190. = variabilis.

One synt. on a card, "Fives Lille / S. Fé. Weiser" print.; "Col. / C. Bruch" print., black frame; "Typus" print. on pale green paper; "222" handwr. on pink paper; "Endalus / variabilis / Hust." handwr. by Bruch, red frame; "ndalus / variabilis" handwr., cut, remainings of "Hust". One synt. missing on a pointed card, "Rep. Argentina / Prov. Buenos Aires / 190_/ C. Bruch" print., black frame; "Col. / C. Bruch" print., black frame; "Typus" print. on pale green paper; "Endalus / variabilis / Hust." handwr. by Bruch, without frame. "MACN-En / 9531 [9532]" print., reverse. Ex CBC.

variabilis [Torcus (Eutorcus)] Hustache 1939: 116-117. Tigre, from Bruch $\mathrm{n}^{\circ} 128$, leg. Bosq $\mathrm{n}^{\circ}$ 103 ; several spec.

variabilis [Torcus]: Wibmer \& O'Brien 1986: 337.
Four synt. (all four?) on cards, on three pins $(1+2+1)$. The 1st pin "Paraná Miní / 1.XI.1915" handwr. by Bruch; "Eutorcus / variabilis / Hust." handwr. by Bruch, without frame; "Eutorcus / variabilis m." handwr. by Hustache?, cut. The 2nd pin, "Rep. Argentina / Prov. Buenos Aires / 14.V.1915 / C. Bruch" print., date handwr., black frame; "Allotorcus / variabilis / Hust. [handwr.] / C. Bruch determ. [print.]" on white paper; "Eutorcus / variabilis / Hust." handwr. by Bruch, green frame. The 3rd pin "Bs. Aires / II.1915" handwr. by Bruch. All three pins "Typus" handwr. by Bruch on pale green paper. To the 3rd pin we add "Torcus / (Eutorcus) / variabilis / Hustache 1939 / Syntypus?" handwr. on red paper."MACN-En / 9607 [9608, 9609, 9610"] print., reverse. Ex CBC.

Note: Tigre refers to the distal (lower) portion of the delta of the Paraná River; Paraná Miní is a stream therein.

variegatus [Menetypus] Hustache 1926: 157158, pl. V: f. 5. Córdoba Prov.: Alta Gracia. La Granja, from Bruch.

variogenitus [Menetypus] Günther in Günther \& Zumpt 1933: 113. Nom. nov. pro variegatus Hustache 1926 non Pierce 1915.

[variogenitus] [Pandeleteius]. Emden \& Emden 1939: 257. = Menetypus, $=$ Pandeleteinus

One synt. on a card, "Alta Gracia. La Granja / Sierras de Córdoba / C. Bruch leg." print.; "Typus", "Foto" print. on pale green paper; "Menetypus / variegatus / Hust." handwr. by Bruch, red frame; "Menetypus / variegatus *" handwr. by Hustache?, cut. "MACN-En / 10010" print., reverse. Ex CBC.

vatius [Helodytes] Kuschel 1952a: 62-63, f. 43. Misiones Prov., ex ABC, holot. $\delta$ in MACN n ${ }^{\circ}$ 51368.

Holot. on a pointed card, "그" print.; "Argentina / Misiones" print.; "Rep. Argentina / A. Breyer" print., with a color stripe indicative of Misiones, "Col./ A. Breyer" print.; "51368" handwr.; "Holotipo" print. on orange paper, "Helodytes / vatius / Kuschel [handwr.] / Kuschel det. 1950 [print.]" on white paper. "MACN-En / 10333" print., reverse.

ventralis [Neochetina] O'Brien 1976: 166, 170-171, f. 4, 10, 13, 15, 16. Paraguay: 3 km E of Ypacaraí (loc. typ.), leg. O'Brien 7.X.1968 on Pontederia lanceolata (Pontederiaceae). Argentina: Formosa Prov.: Gran Guardia IIIII.1953; Pariné II.1974. Bolivia: Santa Cruz: 
Saavedra, Agric. Exper. Stat. XII.1959. Brasil: Mato Grosso: Cáceres XII.1955; Cuiabá III.1972; São Paulo Prov. Castilho X.1962. Paraguay: San Bernardino; Ypacaraí.Lake. Holot., allot., parat. in OBC, parat. in several repositories, including MACN, MLP.

Two parat. on pointed cards, on separate pins, "Paraguay $3 \mathrm{~km}$ E / Ypacaraí X.7.1968" print.; "on Pontederia lanceolata at night. C.W. O'Brien" print.; "Paratype / Neochetina / ventralis / C.W. O'Brien" print. on pale yellow paper. "MACN-En / 10331 [10332]" print., reverse.

Note: Two parat. are at MLP (Morrone \& Loiacono 1994: 19).

vicina [Liobaridia] Hustache 1951a: 10, 11. Tucumán Prov., from Bruch n 253; Salta Prov. Paraguy: Hohenau.

Three synt. on cards, on two pins $(2+1)$. The 1st pin "Rep. Argentina / Prov. Tucuman / V.1897 / C. Bruch" print., date handwr., black frame; "253" handwr. on pink paper. The 2nd pin, "Prov. Salta / Argentin" print., cut. Both pins "Typus" handwr. by Bruch on pale green paper: "Heterosternus / vicinus / Hust." handwr. by Bruch, green frame: "MACN-En / 9631 [9632, 9633, 9634]" print., reverse. Ex CBC.

Note: There is another spec. labeled "Fototypus" by Bruch, from Catamarca, not mentioned in the publication, see the third list, below.

villosulus [Adioristus] Hustache 1926: 186, 189. Jujuy Prov.: Santa Catalina, from Bruch.

villosulus [Scotoeborus]: Kuschel 1949: 34-35, pl. II: f. 7. = A. griseus Germain 1911, nomen nudum.

villosulus [Cylydrorhinus]: Wibmer \& O'Brien 1986: 107. = griseus (Germain) nomen nudum, = villosulus var. unicolor Hustache.

Two synt. on cards, on separate pins, "Jujuy / 10.XI.919. Weiser", "Cueva Iturbe / 2700 m" handwr. by Bruch; "Typus" print. on pale green paper. One of them also "Foto" print. on pale green paper; "Adioristus / villosulus / Hust." handwr. by Bruch, red frame; "Listroderes / villosulus*" handwr. by Hustache? cut. To the other one we add "Adioristus / villosulus / Hustache 1926 / Syntypus" handwr. on red paper. "MACNEn / 9505 [9506]" print., reverse. Ex CBC.

Note: There is another spec. labeled "Typus" from Catamarca Prov., not mentioned in the publication, MACN-En / 9507, see the third list, below.

vulgaris [Hyperodes] Hustache 1926: 203,
206-207. Jujuy Prov., type, from Bruch; Buenos Aires Prov. from Bruch and from Bosq; San Isidro, Paraná, Isla Santiago, Tigre, from Bruch and from Bosq.

vulgaris [Listronotus]: O’Brien 1979: 268.

Two synt. on cards, on one pin, "Rep. Argentina / Prov. Buenos Aires /190_ / C. Bruch" print., black frame; "Typus" print. on pale green paper; "131" handwr. on pink paper; "Hyperodes / vulgaris / Hust." handwr. by Bruch, red frame; "vulgaris Hust." handwr. by Hustache?, cut. "MACN-En / 9508 [9509]" print., reverse. Ex CBC.

Note: Isla Santiago is next La Plata City, Tigre refers to the frontal (lower) portion of the Paraná River Delta.

vulgatus [Neobagous] Hustache 1929: 228-229. $=N$. brasiliensis in litteris. Brasil: Mato Grosso [do Sul] State: Corumbá, from Staudinger, eleven spec. in HUS.

vulgatus [Onychylis]: Wibmer \& O'Brien 1986: 191.

One synt. on a card, "Corumbá / Matt. Grosso" print.; "Typus" handwr. by Bruch on pale green paper; "Neobagous / vulgatus m." handwr. by Hustache?, cut, reverse black. "MACN-En / 9533" print., reverse. Ex CBC.

wittei (sub Wittei) [Listroderes] Hustache 1926: 194-195. In a key.

One synt. on a card, abdomen on another card, a plastic microvial with dissected parts; "Valle Tunel / Dr. Witte" handwr. by Bruch; "Rep. Argentina / Gob. Santa Cruz / 190_ / C. Bruch" print., black frame; "Typus" print. on pale green paper; "Listroderes / Wittei / Hust." handwr. by Bruch, red frame; "Listroderes wittei / Hustache / Det. J.J. Morrone 1988" print. "MACN-En / 9703" print, reverse. Ex CBC.

x-album [Geraeus] Hustache 1924: 96-97. Bolivia: Santa Cruz, types, IX.1917.

$\boldsymbol{x}$-album [Centrinaspis]: Hustache 1938a: 107. X-album [Geraeus], X-album [Centrinaspis]: Blackwelder 1947: 897, 898.

$\boldsymbol{x}$-album [Linogeraeus]: Kuschel 1983: $39 .=$ bipunctatus (Hustache 1950).

One synt. on a card, "Santa Cruz" handwr. by Bruch; "Typus" print. on pale green paper; "Centrinaspis / x-album / Hust." handwr. by Bruch, without frame; "Centrinaspis / $\mathrm{x}$ album m." handwr. by Hustache?, cut. We add "Geraeus / x-album / Hustache 1924 / Syntypus" handwr. on red paper. "MACN-En / 9687" print., reverse. Ex $\mathbf{C B C}$. 


\section{TYPES DEEMED TO BE HOUSED IN THE MUSEUM, BUT NOT FOUND}

\section{SCOLYTIDAE}

porteri (sub Porteri) [Sinophloeus] Brèthes 1923: 434-435, f. 37. Chile: Cautín, on Nothofagus obliqua (Fagaceae).

destructor [Sinophloeus] Eggers 1942: Wood \& Bright 1992: $105 .=$ S. porteri.

One slide "Sinophloeus / Porteri / Brèthes. type! antenne. Cautín, Chile. Porter leg. XI-1921" handwr. by Brèthes; "N $\mathrm{N}^{\circ} 24$ ", handwr., in slide coll. under S20.

Note: Only a slide with an antenna, spec. not found.

\section{CURCULIONIDAE}

atrox [Arodenius] Heller 1921: 32-33, f. 10. Mendoza Prov.: Potrerillos, leg. Carette.

Spec. missing, only the pin, "Arodenius / atrox / Heller" handwr. by Bruch, red frame. Ex CBC.

breyeri (sub Breyeri) [Relistrodes] Brèthes 1910: 210. Misiones Prov., one spec. ex ABC, in MACN.

breyeri [Anchodemus]: Kuschel 1950a: 14.

breyeri [Lixellus]: Burke 1963: 169.

breyeri [Listronotus]: Morrone, Coscarón \& O’Brien 1995: 111.

Note: Holot. not found.

bruchi [Cylydrorhinus] Kuschel 1958a: 248-249, f. 20. Neuquén Prov., holot. $\delta^{\wedge}$, allot. + , parat. in GKC, parat. in MACN, ZMHB.

Note: Parat. not found.

fulvipes [Neogeobyrsa] Brèthes 1910: 214-215. Bolivia.

uniformis [Ochetina] Pascoe 1881: Kuschel 1950b: 113. = Neogeobyrsa fulvipes.

Note: Typi not found.

peruvianus [Mimographus] Brèthes 1920: 3940. Perú: Arequipa.

leporinus [Amphideritus] (Erichson 1847): Wibmer \& O’Brien 1986: 55. Nov combin., nov. synon.

Note: Typi not found.

quadrispinosus (sub4-spinosus) [Probastactes] Brèthes 1910: 209. Bolivia, ex ABC, in MACN.

ferrugatus [Dysphiles] Pascoe 1886: Kuschel 1955: 302. $=$ P. quadrispinosus. ferrugatus [Tetraphysus]: Wibmer \& O'Brien 1986: 86.

Note: Typi not found.

robigo [Polydrusus] Kuschel 1950c: 184-185, f. 1b, photo 3. Chile: Nuble Prov.: Cordillera Chillán; Bio-Bio Prov.: Antuco Vulcan, Laguna Verde $1500 \mathrm{~m}$; Trapa-Trapa $1650 \mathrm{~m}$; Copahue Vulcan, Termas, Malleco Prov.:: Pemehue, Malleco Pond. Holot. (from Chillán), parat. in MNNC, and in several repositories, including MACN, MLP.

roseus [Polydrusus] (Blanchard 1851): Kuschel 1955: $280=$ P. robigo.

Notes: Parat. not found. One parat. is at MLP (Morrone \& Loiacono 1994: 8).

vianai [Acrostomus] Kuschel 1958a: 233-234, f. 3, 4. Chile: Aysén Prov.: Balmaceda 20.II.1957 on Azorella. Argentina: Chubut Prov., W of Lago Blanco, in front of Balmaceda 13.II.1956; Neuquen Prov. Holot. ${ }^{\wedge}$, allot. $\circ$, parat. in GK; parat. in several repositories, including MACN. Notes: Parat. not found.

\section{SPECIMENS LABELED AS TYPES OF NAMES NOT FOUND IN THE BIBLIOGRAPHY AND PROBABLY NOT PUBLISHED}

\section{ANTHRIBIDAE}

gravicornis [Gymnognathus] Jordan

One spec. on a card, "Jatahy / Prov. Goyas. Brésil / Dec. 97 - Janv. 98" print. on green paper; "Cotypus" handwr. by Bruch on pale green paper; "Gymnognathus / gravicornis / Jord. 1931 [handwr. by Bruch] / C. Bruch determ. [print.]" on white paper; " 42877 " handwr. (register book refers to a Hesperiid butterfly); "Gymnognathus / gravicornis / 1937 / Paratypus Jord." handwr. "MACN-En / 10668" print., reverse.

\section{SCOLYTIDAE}

cribricollis [Acorthylus] Brèthes

A slide, "Acorthylus cribricollis Brèthes. type! Bs.As. 11-V-1909. J.B. leg." handwr. on whitish paper. In slide coll. under A3. Ex JB.

\section{porteri [Ernoporus] Brèthes}

A slide with legs and other unidentified parts, "Ernoporus / Porteri Brèthes / type! / Chili. Porter leg. / Quillota" handwr. by Brèthes. Sub E 10. "MACN-En 10672" print. Ex JB. 
strigifrons [Eccoptogaster] Hagedorn

One spec. pinned, abdomen broken, "Rep. Argentina / Prov. Buenos Aires / 190_/ C. Bruch" print., black frame;"Typus" print. on pale green paper; "Eccoptogaster / strigifrons / Haged. tipo" handwr. by Bruch, red frame. "MACN-En / 10627" print., reverse. Ex CBC.

Note: Seemingly not published. Recorded as "in litteris", from Buenos Aires, by Bruch (1915: 428).

\section{CURCULIONIDAE}

aeneosuturalis [Naupactus] Hustache

One spec. pinned, "Misiones" print.; "Typus" print. on pale green paper; "Naupactus / aeneosuturalis / Hust." handwr. by Bruch, red frame; "Naupactus aeneosuturalis *" handwr. by Hustache?, cut. "MACN-En / 9428" print., inverse. $E x \mathbf{C B C}$.

Note: Not recorded by Wibmer \& O'Brien (1986, 1989b).

affinis [Mimographus] Hustache

One spec. on a card, "Rep. Argentina / Pr. Santiago del Estero / 190_ / C. Bruch" print., black frame; "Typus" print. on pale green paper; "Mimographus / affinis / Hust." handwr. by Bruch, red frame; "Mimograp / affinis m." handwr. by Hustache, cut. "MACN-En / 10079" print., reverse. Ex CBC.

Note: Not recorded by Wibmer \& O’Brien (1986, 1989b).

albiventris [Antesis] Hustache

One spec. on a card, "Alta Gracia-La Granja / Sierras de Córdoba / C. Bruch leg." print.; "Typus" handwr. by Bruch on pale green paper; "Antesis / albiventris / Hust." handwr. by Bruch, green frame; "267" handwr. on pink paper. "MACN-En / 9589" print., reverse. Ex CBC.

Note: Not recorded by Wibmer \& O’Brien (1986, 1989b).

alboguttatus [Conothorax] Hustache

Two spec. pinned, "Bompland. Jörgensen" print.; "Typus" ["Cotypus"] print. on pale green paper, one of them also: "Conothorax / alboguttatus Hust." handwr. by Bruch, red frame; "Conothorax / alboguttatus *" handwr. by Hustache?, cut. To the other one we add "Conothorax / alboguttatus / Hustache" handwr. on red paper. "MACN-En / 9995 [9996]" print., reverse. Ex $\mathbf{C B C}$.

Notes: Probably not published fide Wibmer \&
O'Brien (1986: 26). Recorded from Argentina by Bondar (1949: 179).

albomarginalis [Pororhynchus] Hustache

Two spec. on cards, on separate pins, "Rep. Argentina / Pr. Santiago d. Estero / 190_ / C. Bruch" print., black frame; "Typus" print. on pale green paper. One of them also "Foto" print. on pale green paper; "Pororhynchus / albomarginalis / Hust." handwr. by Bruch, without frame; "Pororhynchus albomarginalis" handwr. by Hustache?, cut. To the other we add "Pororhynchus / albomarginalis / Hustache" handwr. on red paper. "MACN-En / 9707 [9708]" print., reverse. $E x \mathbf{C B C}$.

Note: Not recorded by Wibmer \& O'Brien (1986, 1989b).

argentinensis [Coelosternus] Hustache

Seven spec. on cards, on four pins $(2+2+2+1)$, "Alta Gracia. La Granja / Sierras de Córdoba / C. Bruch leg." print.; "Typus" [on pins 1, 2 and 4] "Cotypus" [on pin 3] print. [handwr. by Bruch on pin 3] on pale green paper. The 1st pin also "27" handwr. on pink paper; "Coelosternus / argentinensis / Hust." handwr. by Bruch, red frame; "Coelosternus / argentinensis" handwr. by Hustache? cut. The 3rd pin: also"19292" handwr. (in register book same data); "Coelosternus / argentinensis / Hust." handwr by Bruch, green frame. The 4th pin: also "Foto" print. on pale green paper. To the 2nd \& 4th pins we add "Coelosternus / argentinensis / Hustache / Syntypi? [Syntypus?]" handwr. on red paper. "MACN-En / 10128 [10129 through 10134]" print, reverse. $E x$ CBC.

Note: Not recorded by Wibmer \& O'Brien (1986, 1989).

argentinensis [Lixus] Hustache

Two spec. pinned, "Rep. Argentina / Prov. Buenos Aires / 4.I.1909 [9.1897] / C. Bruch" print., dates handwr., black frames; "Typus" ["Cotypus"] print. on pale green paper, the "typus" also "Lixus argentinensis Hust." handwr. by Bruch, red frame; "argentinensis n. sp." handwr. by Hustache?, cut; " 183 " handwr. on pink paper. To the "cotypus" we add "Lixus argentinensis Hustache" handwr. on red paper. "MACN-En / 9993 [9994]" print., reverse. Ex CBC.

Note: Not recorded by Wibmer \& O'Brien (1986, 1989b).

astans [Caneorhinus] Kuschel

One spec. pinned, "Sta. Cruz. / L. Viedma 
23.2.48 / Wittmer" handwr.; "Paratipo" print. on yellowish orange paper, "Caneorhinus / astans n. sp. [handwr.] / Kuschel det. 1955 [print.]". on white paper. "MACN-En / 10256" print., reverse. Ex CBC.

Note: Not recorded by Wibmer \& O’Brien (1986, 1989b).

auricollis [Phyrdenus] Hustache

One spec. on a card, "Rep. Argentina / Gob. Formosa / 190 / C. Bruch" print., black frame; "Typus" print. on pale green paper; " 6 " handwr. on pink paper; "Phyrdenus / auricollis / Hust." handwr. by Bruch, red frame; "Phyrdenus / auricollis m'" handwr. by Hustache?, cut. "MACNEn / 10093" print., reverse. Ex CBC.

Note: Probably a nomen nudum, original publication not found; cited from Argentina by Bosq (1943: 56), fide Wibmer \& O’Brien 1986: 27.

boliviensis [Heterosternus] Hustache

One spec. on a pointed card, "Cuatro Ojos. Bolivia" print.; "Typus" handwr. by Bruch on pale green paper; "Heterosternus / boliviensis/ Hust." handwr. by Bruch, without

frame; "Heterosternus / boliviensis m." handwr. by Hustache?, cut. "MACN-En / 9622" print., reverse. $E x \mathbf{C B C}$.

Note: Not recorded by Wibmer \& O’Brien (1986, 1989b).

boliviensis [Metoponeurys] Hustache

One spec. on a card, "Corrientes / San Roque / J. Bosq II.1920" handwr. by Bruch; One spec. on a card, "Rep. Argentina / Prov. Corrientes / 190 / C. Bruch" print., black frame. Both: "Cotypus" print. on pale green paper; "Metoponeurys / boliviensis Hustache" handwr. by Bruch, red frame. "MACN-En / 10080 [10081]" print., reverse. Ex CBC.

Notes: Probably a nomen nudum, original publication not found fide Wibmer \& O'Brien (1986: 27). Cited by Bosq (1943: 54) from Argentina. This species was described by Hustache (1947b: 117) as Pantomorus conirostris, type locality Corrientes Province: San Roque, and there is coincidence with the date of collecting. The current valid name is Aramigus conirostris (Hustache). See the revision of Aramigus (Lanteri \& Díaz 1994).

bruchi $[$ Eurylobus] Hustache

One spec. pinned, "Misiones" print. on whitish paper; "Typus" print. on pale green paper; "Eurylobus / Bruchi Hust." handwr. by Bruch, red frame; "Eurylobus Bruchi" handwr. by Hustache?, cut. "MACN-En / 10164" print., reverse. $E x$ CBC.

Note: Not recorded by Wibmer \& O'Brien (1986, 1989b).

bruchi $[$ Lixus $]$ Hustache

Four spec. pinned, "Hualfin Catam. / Weiser" handwr. by Bruch; "Typus" [on two], "Cotypus" [on two] print. on pale green paper; three of them also "Lixus / Bruchi / Hust." handwr. by Bruch, red frame [without frame on two]; one of these three "Lixus Bruchi" handwr. by Hustache?, cut; "182" handwr. on pink paper; another one of these three "Foto" print. on pale green paper; "Col. / C. Bruch" print., black frame. We add "Lixus / bruchi / Hustache" handwr. on red paper. "MACN-En / 9997 [9998]" print., reverse. Ex CBC.

Note: Not recorded by Wibmer \& O'Brien (1986, 1989b).

\section{bruchi $[$ Wagneriella] Hustache}

One spec. on a card, "Rep. Argentina / Prov. Tucuman / II.1906 / C. Bruch" print., date handwr., black frame; "Typus" print. on pale green paper; "Wagneriella / Bruchi / Hust." handwr. by Bruch, red frame; "Wagneriella / Bruchi * olim" handwr. by Hustache?, cut. "MACN-En / 9989" print., reverse. $E x$ CBC.

Notes: Not recorded by Wibmer \& O'Brien (1986, 1989b). Hustache (1923) published the description of two species of Wagneriella, W. lineata and W. albula, later synonymized by Lanteri (1982) as they correspond to female and male of the same species, with great sexual dimorphism. The spec. at MACN is a male. The author cited type material from Bolivia and from Santiago del Estero Prov. (Argentina) but not from Tucumán.

cinereosquamis [Heilipus] Heller

One spec. pinned, "Rep. Argentina / Prov. Catamarca / II.1910 / C. Bruch" print., date handwr., black frame; "Typus" print. on pale green paper; "Heilipus cinereo-squamis Heller i. litt." handwr. by Bruch, red frame; "lipus cinereosquamis mihi" handwr. by Heller, cut, with remainings of "K.M. Heller" print. "MACN-En / 10001" print., reverse. Ex CBC.

Note: Not recorded by Wibmer \& O'Brien (1986, 1989b).

curtipennis [Naupactus] Hustache

One spec. on a card, "Rep. Argentina / Gob. Chaco / 189_ / C. Bruch" print., black frame; 
"Typus" print. on pale green paper; "Naupactus / curtipennis / Hust." handwr. by Bruch, red frame; "Pantomorus / carinirostris / Hustache / det. Lanteri 90", handwr. on white paper. "MACN-En / 9433" print., reverse. Ex CBC.

Notes: Not published, fide Lanteri in litteris 15.V.1991. Not recorded by Wibmer \& O'Brien (1986, 1989b). The spec. was identified in 1990 by Lanteri as Pantomorus carinirostris Hustache 1947.

cyrillae [Scalaventer] Howden

Two spec. on pointed cards, on separate pins, "Jamaica. 4000 / Hardwar Gap VIII-25-1966 / A.T. Howden" print.; "On Cyrilla racemiflora" print.;"Paratype, Scalaventer / cyrillae A.T. Howden" print. on yellow paper. "MACN-En / 10381 [10382]" print., reverse.

Note: Not recorded by Wibmer \& O’Brien (1986, $1989 b)$, not even the genus.

dentinus [Heilipus] Hustache

One spec. pinned, "Rep. Argentina / Prov. Cordoba / 6.II.1901 / C. Bruch" print., date handwr., black frame; "Typus" print. on pale green paper; "Hilipus / dentinus / Hust." handwr. by Bruch, red frame; "dentinus* Faust in litt. m." handwr. by Hustache?, cut; "dentinus Faust i.c. [handwr.] / Determ. K.M. Heller [print.]" on white paper, cut. "MACN-En / 10002" print., reverse. Ex CBC

Note: Not recorded by Wibmer \& O’Brien (1986, 1989b).

elongatulus [Naupactus] Hustache

One spec. pinned, "Rep. Argentina / Prov. Salta / 190 / C. Bruch" print., black frame; "Typus" print. on pale green paper; "Naupactus / elongatulus / Hust." handwr. by Bruch, red frame; "Naupactus / elongatulus" handwr. by Hustache?, cut. One spec. pinned, "Rep. Argentina / Gob. Chaco / 190_ / C. Bruch" print., black frame; "Foto", "Typus" print. on pale green paper; we add "Naupactus / elongatulus / Hustache" handwr. on red paper. "MACN-En / 9434 [9435]" print., reverse. Ex CBC.

Note: Not recorded by Wibmer \& O’Brien (1986, 1989b).

elongatus $[$ Analcis $]$ Hustache

Five spec. on cards, on three pins $(1+2+2)$. The 1st pin: "Rep. Argentina / Prov. Santa Fe / V.1913 / C. Bruch" print., date handwr., black frame; "Typus" print. on pale green paper; " 8 " handwr. on pink paper; "Analcis / elongatus /
Hust." handwr. by Bruch, red frame; "Analcis / elongatus" handwr. by Hustache?, cut. The 2nd \& 3rd pins "Rep. Argentina / Prov.Buenos Aires / 6.I.1905 [190_] / C. Bruch" print., date handwr., black frame; "Cotypus" print. on pale green paper, one of them also "Analcis / elongatus / Hustache" handwr. by Bruch, without frame; to the other ones we add "Analcis / elongatus / Hustache" handwr. on red paper. "MACN-En / 9578 [9579 through 9582]" print., reverse. Ex CBC.

Note: Not recorded by Wibmer \& O'Brien (1986, 1989b).

elongatus [Rhyssomatus] Hustache

Two spec. on cards, on one pin, "Rep. Argentina / Prov. Buenos Aires / 190 / C. Bruch" print., black frame; "Typus" print. on pale green paper; "10" handwr. on pink paper; "Rhyssomatus / elongatus / Hustch." handwr. by Bruch, red frame; "Rhyssomatus / elongatus m.*" handwr. by Hustache?, cut. "MACN-En / 10139 [10140]" print., reverse. Ex CBC.

Notes: Probably a nomen nudum, description not found, fide Wibmer \& O'Brien (1986: 27). Cited by Viana (1937: 107) from Martín García Island.

fulvoaureus [Naupactus] Hustache

One spec. pinned, "Rep. Argentina / Gob. Misiones /190_ / C. Bruch" print., black frame; "Typus" print. on pale green paper; "Naupactus / fulvoaureus / Hust." handwr. by Bruch, red frame; "Naupactus / fulvoaureus" handwr. by Hustache?, cut. "MACN-En / 9436" print., reverse. $E x$ CBC.

Notes: Probably a nomen nudum, description not found, fide Wibmer \& O'Brien (1986: 27). Cited from Argentina by Bosq (1943: 55).This species was described as Naupactus imbellis Hustache 1947: 89; this author stated that the name imbellis corresponds to $N$. fulvoaureus in litteris.

\section{globulipennis [Cyphus] Hustache}

Two spec. pinned, "Rep. Argentina / Prov. La Rioja / 1.1904 / C. Bruch" print., date handwr., black frame; "Typus" ["Cotypus"] print. on pale green paper; one of them also "Cyphus / globulipennis / Hust." handwr. by Bruch, red frame; "Cyphus / globulipennis *" handwr. by Hustache?, cut; the other one also "Heller vid." handwr.; we add "Cyphus / globulipennis / Hustache" handwr. on red paper. One spec. pinned, "Rep. Argentina / Pr. Santiago d. Estero / 190 / C. Bruch" print., black frame; "Cotypus" print. on pale green paper; "Cyphus / globulipen- 
nis / Hust." handwr. by Bruch, red frame; "ovalipennis Hust. [handwr. with pencil] / Kuschel det. 1957 [print.]". "MACN-En / 10086 [10087, 10088]" print., reverse. Ex CBC.

Note: Not recorded by Wibmer \& O'Brien (1986, 1989b). This species was described by Hustache (1938) as Neocyphus ovalipennis. The current combination is Cyrtomon ovalipennis (Hustache), see Lanteri (1990b).

\section{interruptus [Cophes] Brèthes}

One spec. on a card, head missing, remounted, it has a pinhole, "Beni. Bolivia" handwr. on green paper, reverse white; "Repreparo / M. Viana / I-1960" handwr.; "Cophes / interruptus Brèthes" handwr. by Brèthes on whitish paper. "MACNEn / 10255" print., reverse. Ex JB.

Note: Not recorded by Wibmer \& O'Brien (1986, 1989b).

ligneolus [Platyomus] Heller

One spec. pinned, "Bompland / Jörgensen" print.; "Det. K.K. Heller" print.; "Cotypus" print. on pale green paper; "Platyomus / ligneolus / Heller i. litt." handwr. by Bruch, red frame; "perlepidus Boh. [handwr. with pencil] / Kuschel det. 1957 [print.]". "MACN-En / 9987” print., reverse. $E x \mathbf{C B C}$.

Note: Not recorded by Wibmer \& O'Brien (1986, 1989b).

lizeri $[$ Lixus] Hustache

One spec. pinned, "Rep. Argentina / Prov. Santa $\mathrm{Fe} /$ V.1913 / C. Bruch" print., date handwr., black frame; "Rosario / Hubrich leg." handwr. by Bruch; "184" handwr. on pink paper; "Cotypus" print. on pale green paper; "Lixus / Lizeri / Hust." handwr. by Bruch, red frame; "Lizeri m." handwr. by Hustache?, cut. "MACN-En / 9992" print., reverse. Ex CBC.

Note: Not recorded by Wibmer \& O'Brien (1986, 1989b).

longehirtus [Naupactus] Hustache One spec. pinned, "Rep. Argentina / Prov. Mendoza / 190_ / C. Bruch" print., black frame; "Typus" print. on pale green paper; "Naupactus / longehirtus / Hust." handwr. by Bruch, red frame; "Naupactus / longehirtus" handwr. by Hustache?, cut; "Pantomorus ruizi (Brèthes) / Det. A.A. Lanteri 1988" print. on white paper. "MACN-En / 9437" print., reverse. Ex CBC.

Notes: Not recorded by Wibmer \& O’Brien (1986, 1989b). The spec was dentified and labeled in 1988 as Pantomorus ruizi (Brèthes) by Lanteri. It was described by Brèthes (1925) as Mimographus ruizi. The current name is Pantomorus ruizi (Brèthes). It is a variable species with a quite extensive synonymy (Morrone \& Lanteri 1991).

\section{marmoratus [Heilipus] Hustache}

One spec. pinned, "Sgo. del Estero / Rio Salado / Wagner col." print.; "Cotypus" print. on pale green paper; "Heilipus / marmoratus / Hustch." handwr. by Bruch, red frame. "MACN-En / 10003" print., reverse. Ex CBC.

Note: Not recorded by Wibmer \& O'Brien (1986, 1989b).

minutus [Heterosternus] Hustache

One spec. on a card, "Corrientes I.1921. Colec. De Carlo" handwr.; "Heterosternus / minutus / Hust." handwr., by Bruch, without frame; "Heterosternus / minutus m." handwr. by Hustache?, cut. One spec. on a card, "Santa Fé" handwr. by Bruch. One spec.

on a card, "San Roque. Corr. Bosq" handwr. by Bruch. All three: "Typus" handwr. by Bruch on pale green paper. To the 2nd \& 3rd ones we add "Heterosternus / minutus / Hustache" handwr. on red paper. "MACN-En / 9619 [9620, 9621]" print., reverse. Ex CBC.

Notes: Probably a nomen nudum, description not found, fide Wibmer \& O'Brien (1986: 27). Cited by Viana (1937: 107) from Martín García Island.

\section{minutus [Menetypus] Hustache}

One spec. on a card, "Rep. Argentina / Prov. Tucuman / II.1906 / C. Bruch" print, date handwr., black frame; "Typus" print. on pale green paper; "Menetypus / minutus / Hust." handwr. by Bruch, red frame; "Menetypus / minutus m." handwr. by Hustache?, cut. "MACN-En / 9984" print., reverse, $E x$ CBC.

Note: Not recorded by Wibmer \& O'Brien (1986, 1989b).

missionensis [Chalcodermus] Hustache One spec. on a card, "Rep. Argentina / Prov. Santa Fe / 190 / C. Bruch" print., black frame; "Rosario / Hubrich" handwr. by Bruch; "Typus" print. on pale green paper; "177" handwr. on pink paper;"Chalcodermus / missionensis / Hust." handwr. by Bruch, red frame; "Chalcodemus / missionensis" handwr. by Hustache?, cut. "MACN-En / 10142" print., reverse. Ex CBC. Note: Not recorded by Wibmer \& O'Brien (1986, 1989b).

missionis [Oxycorynus] Kuschel 
Two spec., $\hat{\gamma}$, + , on cards, on separate pins, "Loreto. exp. St. / Missiones Arg. / Dr. A. Ogloblin" print.; "Col. / C. Bruch" print., black frame; "Holotype $\widehat{\overbrace{}}$ [Paratype $\odot]$ / Oxycorynus / missionis [handwr.] / Kuschel '92 [print.]" on red [blue] paper; "Oxycorynus / missionis / Kuschel [handwr.] / det. G. Kuschel / 1992 [print.]" on white paper. "MACN-En / 9981 [9982]" print., reverse. Ex CBC.

Note: Not recorded by Wibmer \& O’Brien (1986, 1989b).

\section{niger [Opseotapinotus] Bruch}

One spec. on a card, "Rep. Argentina / Prov. Buenos Aires / 190_ / C. Bruch" print., black frame; "San Blas" handwr. by Bruch; "Typus" print. on pale green paper; "Opseotapinotus / niger / Bruch [handwr. by Bruch] / C. Bruch determ. [print.]" on white paper, black frame. "MACN-En / 10067" print., reverse. Ex CBC.

Note: Not recorded by Wibmer \& O’Brien (1986, 1989b).

ovalipennis [Naupactus] Hustache

ruizi [Pantomorus] (Brèthes): Morrone \& Lanteri 1991: 18-22, pl. 1-4. Nov. comb.; = subvittatus Fairmaire \& Germain 1861 non Boheman 1840; = subvittatulus Emden 1936: 23 nomen novum; = ovalipennis Hustache 1943 nomen nudum, syn. nov.; = Asynonychus variabilis and var. intermedius Hustache 1947b: 139.

One spec. pinned, "Rep. Argentina / Gob. Rio Negro/ 190_ / C. Bruch" print., black frame; "Typus" print. on pale green paper; "Naupactus / ovalipennis / Hust." handwr. by Bruch, red frame; "Naupactus / ovalipennis *" handwr. by Hustache?, cut. One spec. pinned, "Rep. Argentina / Prov. Buenos Aires / 190 / C. Bruch" print., black frame; "San Blas" handwr. by Bruch; "Foto", "Typus" print. on pale green paper. One spec., pinned "Rio Colorado" handwr. by Bruch; "Typus" print. on pale green paper. One spec., pinned "Rio Colorado" handwr. by Bruch; "Cotypus" print. on pale green paper; "Naupactus / ovalipennis / Hust." handwr. by Bruch, without frame. One spec. pinned, "Rep. Argentina / Prov. Buenos Aires / 190_/ C. Bruch" print., black frame; "Cotypus" print. on pale green paper; "Naupactus / ovalipennis / Hust." handwr. by Bruch, without frame. To the 2nd \& 3rd ones we add "Naupactus / ovalipennis / Hustache" handwr. on red paper. "MACN-En / 9438 [9439, 9440, 9441, 9442]" print., reverse. Ex CBC.

Notes: Not recorded by Wibmer \& O'Brien (1986, $1989 b$ ); recorded as a synonym (despite it is a no- men nudum) of Pantomorus ruizi (Brèthes) by Morrone \& Lanteri (1991: 18). Naupactus longehirtus Hustache is another nomen nudum for Pantomorus ruizi.

pictipennis [Mimographus] Hustache One spec. on a card, "Rep. Argentina / Prov. Buenos Aires / 190 / C. Bruch" print., black frame; "Typus" print. on pale green paper; "Mimographus / pictipennis / Hust." handwr. by Bruch, red frame; "Mimographus / pictipennis *" handwr. by Hustache?, cut. "MACN-En 10078" print., reverse. Ex CBC.

Note: Not recorded by Wibmer \& O'Brien (1986, 1989b). It was described by Hustache (1947b: 49) as Pantomorus pictipennis, a junior synonym (color variety) of Pantomorus taeniatulus Berg 1881. Currently Atrichonotus taeniatulus (Berg) (Lanteri \& O’Brien 1990).

posticeguttatus [Centrinaspis] Hustache Three spec. on cards, on one pin, "Rep. Argentina / Prov. Buenos Aires / 10.X.1906 / C. Bruch" print., date handwr., black frame; "Typus" handwr. by Bruch on pale green paper; "260" handwr. on pink paper;"Centrinaspis / foveatus / Hust." handwr. by Bruch, green frame; "Centrinaspis / posticeguttatus / Hust. [handwr. by Bruch] / C. Bruch determ. [print.]" on white paper. "MACN-En / 9662 [9663, 9664]" print., reverse. Ex CBC.

Notes: Probably a nomen nudum, description not found, fide Wibmer \& O'Brien (1986: 26). Recorded from Paraguay by Jacob (1937: 154).

quadrivirgulatus [Conotrachelus] Hustache One spec. on a card, "Rep. Argentina / Prov. Mendoza / 190_ / C. Bruch" print., black frame; "Typus" print. on pale green paper; " $4 . a$ " handwr. on pink paper; "Conotrachelus / quadrivirgulatus / Hustch." handwr. by Bruch, red frame; "Conotrachelus / bisignatus Boh." handwr. by Bruch, red frame. "MACN-En / 10089" print., reverse. Ex CBC.

Note: Not recorded by Wibmer \& O'Brien (1986, 1989b).

rugipennis [Naupactus] Hustache One spec. pinned, "Rep. Argentina / Prov. Mendoza / I.1900 / C. Bruch" print, date handwr., black frame; "Foto", "Typus" print. on pale green paper; "Naupactus / rugipennis / Hust." handwr. by Bruch, red frame; "Naupactus / rugipennis" handwr. by Hustache?, cut. "MACN-En / 9443" print., reverse. Ex CBC. 
Note: Not recorded by Wibmer \& O’Brien (1986, 1989b). The species was later described as Naupactus rugosus Hustache 1947b: 93 whose original publication says that there are types from Mendoza (Cacheuta 21-IV-1907) and Catamarca (3-XI-1910) at Hustache coll., and MNHN.

saladensis [Heilipus] Hustache

One spec. pinned, "Paraguay / Sa. Trinidad / X.1913" print., date handwr.; "Cotypus" print. on pale green paper; "Heilipus / saladensis / Hust." handwr. by Bruch, red frame; "Heilipus / saladensis" handwr. by Hustache?, cut. "MACNEn / 10007" print., reverse. Ex CBC.

Note: Not recorded by Wibmer \& O’Brien (1986, 1989b).

seriegranulatus [Heilipus] Hustache

One spec. pinned, "Tandil" handwr. by Bruch; "Typus" print. on pale green paper; "Hilipus / seriegranulatus / Hust." handwr. by Bruch, red frame; "seriegranulatus * m." handwr. by Hustache?, cut. "MACN-En / 10006" print., reverse. Ex CBC.

Note: Not recorded by Wibmer \& O’Brien (1986, 1989b).

sulfureolateralis [Naupactus] Hustache

One spec. pinned, "Rep Argentina / Pr. Santiago d. Estero / 190_ / C. Bruch" print., black frame; "Typus" print. on pale green paper; "Naupactus / sulfureolateralis / Hust." handwr. by Bruch, red frame; "Naupactus / sulfureolateralis" handwr. by Hustache?, cut. "MACN-En / 9444" print., reverse. $E x \mathbf{C B C}$.

Notes: Not recorded by Wibmer \& O'Brien (1986, 1989b). The species is sometimes misidentified as N. sulfurifer Pascoe.

sylvaticus [Heilipus] Hustache

One spec. pinned, "Sgo. del Estero / Rio Salado /Wagner Col." print.; "Cotypus" print. on pale green paper; "Heilipus / sylvaticus / Hustch." handwr. by Bruch, red frame. "MACN-En / 10005" print., reverse. Ex CBC.

Note: Not recorded by Wibmer \& O’Brien (1986, 1989b).

sylvicola [Conotrachelus] Hustache

One spec. on a card, "Rep. Argentina / Prov. Buenos Aires / 25.IX.1904 / C. Bruch" print., date handwr., black frame; "Typus" print. on pale green paper; "Conotrachelus / sylvicola / Hustch." handwr. by Bruch, red frame; "Conotrachelus / sylvicola m." handwr., cut. "MACN-En / 10090" print., reverse. Ex CBC.
Note: Not recorded by Wibmer \& O'Brien (1986, 1989b). Champion (1904: 408) described another species from Panamá as Conotrachelus silvicola.

vulgaris [Heilipus] Hustache

One spec. pinned, "Misiones. Bompland" print.; "Typus" print. on pale green paper; "Hilipus vulgaris Hust." handwr. by Bruch, red frame; "vulgaris m." handwr. by Hustache?, cut. "MACN-En / 10004" print., reverse. Ex CBC.

Note: Not recorded by Wibmer \& O'Brien (1986, 1989b).

\section{wagneri [Tiphaura] Hustache}

One spec. pinned, "Rep. Argentina / A. Breyer" print., greasy (despite having been washed with bencene, the original color of the stripe was not made clearly distinguishable, but seemingly is indicative of La Rioja Prov.); "Typus" print. on pale green paper; "216" handwr. on pink paper; "Tiphaura / Wagneri" handwr., cut. One spec. pinned, "Alta Gracia. La Granja / Sierras de Córdoba 17.1.927 [?] / C. Bruch leg." print., date handwr., hardly legible; "Cotypus" print. on pale green paper; "Col. / C. Bruch" print., black frame. We add "Tiphaura / wagneri Hustache" handwr. on red paper. "MACN-En / 9709 [9710]" print., reverse. Ex CBC.

Note: Not recorded by Wibmer \& O'Brien (1986, 1989b).

willineri [Tachygonus] Viana

One spec. on a card, head and prothorax missing, " $>$ ” print.; "Chapare (Yungas). 1.49. Suilar" print. on gray paper; "Prep. $\mathrm{n}^{\circ} 3173$ " handwr.; "Tachygonus / willineri Viana / Holotipo $\delta$ [handwr.] / Museo Argentino de Ciencias Naturales [print.] [obverse] / M. Viana X-1950 [reverse]" on white paper, black frame. "MACN-En / 10406" print., reverse. One slide "Genital $\delta$ del Holotipo. Bolivia. Chapare.Yungas. I-1949. Leg. Suilar. N ${ }^{\circ} 3173$ "; "Tachygonus willineri Viana. Genital ô del Holotipo. Det. M. Viana. X-1950 [handwr.] / Museo Arg. C. Naturales. Sección Entomología [print.]" on white paper. In slide coll. $\mathrm{n}^{\circ} \mathrm{T} 100$.

Note: Not recorded by Wibmer \& O’Brien (1986, 1989b).

\section{SPECIMENS OF DUBIOUS TYPE STATUS, BECAUSE THE INFORMATION ON THE LABELS DOES NOT AGREE WITH THAT OF THE ORIGINAL PUBLICATION.}

\section{CURCULIONIDAE}


aeneotinctus [Linomadarus] Hustache 1940b: 281-282. Bolivia: Yungas: Nor-Yungas, Caranavi V.1931, leg. Denier. Tucumán, ex Bruch.

discalis [Linomadarus] Casey 1922: Kuschel 1983: $39=$ L. aeneotinctus.

One spec. on a card, "Misiones" print.; "Typus" handwr. by Bruch on pale green paper; "Linomadarus / aeneotinctus / Hust." handwr. by Bruch, green frame; "252" handwr. on pink paper. "MACN-En / 4599" print., reverse. Ex CBC. Notes: Misiones was not mentioned in the description. The spec. is labeled only by Bruch, not by Hustache. One synt. is recorded at the main list, above.

affinis [Geraeus] Hustache 1924: 99. Bolivia: Cuatro Ojos. XI.1917, type. Two further spec. perfectly identical. May be a meridional form of lineellus Leconte $=$ dolus Schaufuss.

affinis [Centrinaspis]: Hustache 1938a: 101. quadrivittatus [Linogeraeus] (Fabricius 1801): Kuschel 1983: 39. = affinis (Hustache), = australis (Casey), = coroicana (Hustache 1950), = flaveolus (Boheman 1844), = peruviana (Hustache 1950).

One spec. on a card, "Rep. Argentina / Prov. Tucuman / 190_ / C. Bruch" print., black frame; One spec. on a card, "Trinidad / B.W. Indies" print. ; "Centrinaspis / affinis m." handwr. by Hustache?, cut. Both: "Typus" handwr. by Bruch on pale green paper; "Centrinaspis / affinis / Hust." handwr. by Bruch, green frame [without frame]. We add "Geraeus / affinis / Hustache 1924" handwr. on red paper. "MACN-En / 9651 [9652]" print., reverse. Ex CBC.

Note: Neither Tucumán nor Trinidad were mentioned in the original description.

affinis [Neochetina] Hustache 1926: 222-223. Buenos Aires Prov., $\hat{o}$, o f from Bruch.

One spec. on a card, "Rep. Argentina / Gob. Misiones / 190_ / C. Bruch" print., black frame; "Typus" print. on pale green paper; "Neochetina / affinis / Hust." handwr. by Bruch, red frame; "145" handwr. on pink paper. "MACN-En / 9510" print., reverse. Ex CBC.

Notes: Misiones was not mentioned in the original publication. The spec. is labeled only by Bruch, not by Hustache.

alba [Opseobaris] Hustache 1951b: 9. Corrientes Prov.: San Roque, from Bosq; [Santiago del Estero Prov.]: Rio Salado.

alba [Opseobaris] Bondar 1942: Kuschel 1955: $274=$ O. alba Hustache.
Two spec. on cards, on one pin, "Rep. Argentina / Prov. Cordoba / 190_/ C. Bruch" print., black frame; "Cotypus" handwr. by Bruch on pale green paper; "19276" handwr. (in register book same data); "Strangylotes / albus / Hust." handwr. by Bruch, green frame. "MACN-En 10940 [10041]". Ex CBC.

Notes: Córdoba was not mentioned in the original description. Three synt. from Santiago del Estero and Corrientes Prov. are recorded in the main list, above. Ex CBC.

albofasciatus [Palistes] Hustache 1951b: 3132. "Masda III.1921 Weiser", from Bruch n 124. One synt.? on a card, "Fte. Quemado / Catam. Wolters" handwr. by Bruch; "Typus" handwr, by Bruch on pale green paper; "Palistes / albofasciatus / Hust." handwr. by Bruch, green frame. "MACN-En / 9590" print, reverse. Ex CBC.

Note: The type condition is dubious, as the collecting locality differs from the published one; the spec. is labeled only by Bruch, not by Hustache.

albolateralis [Pororrhynchus] Hustache 1924: 159. Bolivia:Yacuiva VIII.1917, tipo.

Seven spec. [parat.?] on cards, on three pins $(2+2+3)$. The 1st pin "Pto. Quemado / Catam. Wolters" handwr. by Bruch; "Typus" print. on pale green paper; "Pororrhynchus / albolateralis / Hust." handwr. by Bruch, red frame; "Pororrhynchus / albolateralis" handwr. by Hustache?, cut; " 201 " handwr. on pink paper. The 2nd \& 3rd pins: "Chiquimil / Catam. Wolters" handwr. by Bruch; "Cotypus" print. [handwr. by Bruch] on pale green paper; the 3rd pin also "19279" handwr., red frame (in register book same data); "Pororrhynchus / albolateralis / Hust." handwr. by Bruch, green frame. To the 2nd pin we add "Pororhynchus / albolateralis / Hustache 1924" handwr. on red paper. "MACNEn / 10172 [10173 through 10178]" print., reverse. $E x$ CBC.

Note: Only Bolivia was mentioned; the "Cotypus" labels suggest they were intended as parat., but the labels are written only by Bruch. The type condition is dubious.

albus [Menetypus] Hustache 1923: 287-288. Chaco de Santiago del Estero Prov., type in HUS; Bolivia: Villa Montes XI.1917. Brasil: Rio de Janeiro State: Mendès, $92 \mathrm{~km}$ from Rio de Janeiro, in HUS.

albus [Pandeleteius]: Wibmer \& O'Brien 1986: 69.

One spec. on a card, "Rep. Argentina / Prov. Tucuman / II.1906 / C. Bruch" print., date hand- 
wr., black frame; "Typus" print. on pale green paper; "Menetypus / albus / Hust." handwr. by Bruch, red frame: "Menetypus / albus" handwr. by Hustache?, cut. "MACN-En / 9988" print., reverse. $E x$ CBC.

Note: Tucumán was not mentioned in the description.

angulithorax [Naupactus] Hustache 1947b: 64-65. Tucumán Prov. §ิ V.1907, ex Bruch ${ }^{\circ}$ 49; San Juan Prov.: Carpintería ㅇ I.1907, from Bosq.

Two spec. pinned, "Rep. Argentina / Prov. Salta / 190_ / C. Bruch" print., black frame; "Heller vid." handwr.; "Typus" print. on pale green paper; "Naupactus / angulithorax / Hust." handwr. by Bruch, red frame; "Naupactus / angulithorax *" handwr. by Hustache?, cut. "MACN-En / 9429" print., reverse. Ex CBC.

Note: Salta was not mentioned in the description.

argentinensis [Hyphantus] Hustache 1926: 156, pl. I: f. 1. Misiones Prov.: Alto Paraná: San Ignacio, leg. Wagner; Misiones, from Bruch; Buenos Aires, from Bruch.

One synt. on a card, "Rep. Argentina / Pr. Santiago d. Estero /190_ / C. Bruch" print., black frame; "Foto", "Typus" print. on pale green paper. We add "Hyphantus / argentinensis / Hustache 1926" handwr. on red paper. "MACNEn 10015” print., reverse. Ex CBC.

Note: Santiago del Estero was not mentioned in the description. Two synt. from Buenos Aires Prov. are recorded in the main list, above.

argentinica [Tonesia] Hustache 1951b:18. Misiones Prov. VIII.1904, from Bruch $\mathrm{n}^{\circ} 125$; Santa Fe Prov.: Fives Lille, leg. Weiser, from Bruch $\mathrm{n}^{\circ} 225$. "= Lyterius argentinensis $m$. in litt."

Two spec. on cards, on one pin, "Rep. Argentina / Gob. Chaco / 2.1898 / C. Bruch" print., date handwr., black frame; "Typus" handwr. by Bruch on pale green paper; "Tonesia / argentinensis / Hust." handwr. by Bruch, green frame; "125" handwr. on pink paper. "MACN-En / 9593 [9594]" print., reverse. Ex CBC.

Note: Chaco was not mentioned in the description. Four synt. from Misiones and Santa Fe are recorded in the main list, above.

argentinicus [Cholus] Heller 1906: 16-17. Chaco Prov. XII.1897 from Bruch, in SMTD sub 17993; = Richteri Faust in coll.

One spec. pinned, "Rep. Argentina / Gob.
Misiones / 12.1897 / C. Bruch" print., date handwr., black frame; "K.M. Heller" print.; "Typus" print. on pale green paper; "Cholus argentinicus / Heller. Tipo" handwr. by Bruch, red frame; "Ptinus / hirtithorax Pic / var. désiré" handwr., cut [rotulation error?] "MACN-En 10160" print., reverse. Ex $\mathbf{C B C}$.

Notes: Misiones was not mentioned in the original description. A puzzling case, seemingly due to multiple rotulation errors. A synt. from Chaco Prov. is recorded under MACN-En / 10161 in the main list, above.

argentinicus [Hilipus, sic, pro Heilipus] Heller 1921: 33-34, f. 11. Córdoba Prov. 14.II.1901; Catamarca and Tucumán Prov., from Bruch. argentinicus [Heilipodus]: Kuschel 1955: 295.

Two spec. pinned, "Rep. Argentina / Prov. Mendoza / 190_ / C. Bruch" print., black frame; "Typus" print. on pale green paper, one of them "Hilipus / argentinicus / Heller" handwr. by Bruch, red frame; to the other one we add "Hilipus / argentinicus / Heller 1921 / Syntypus?" handwr. on red paper. "MACN-En / 9690 [9692]" print., reverse. Ex CBC.

Notes: Mendoza was not mentioned in the original description. A synt. from Catamarca is recorded at the main list, above.

aureomicans [Geraeus] Hustache 1924: 100101. Corrientes Prov.: San Roque XI.1920, one spec. from Bosq, in HUS. Bolivia: Lagunillas XI.1917, one spec.

Five spec. on cards, on three pins $(3+1+1)$. The 1st pin: "Rep. Argentina / Prov. Tucuman / IV.1897 / C. Bruch" print., date handwr., black frame; "Typus" handwr. by Bruch on pale green paper; "Brachygeraeus / aureomicans / Hust." handwr. by Bruch, green frame; " 263 " handwr. on pink paper. The 2nd pin: "Rep. Argentina / Prov. Tucuman / 15.XII.1900 / C. Bruch" print., date handwr., black frame; "Typus" handwr. by Bruch on pale green paper. The 3rd pin: "Tucuman / 15.XII.1900 / C.B." handwr. by Bruch; "Typus" handwr. by Bruch on pale green paper; "Brachygeraeus / aureomicans / Hust." handwr. by Bruch, without frame; "Brachygereus (sic) / aureomicans m." handwr. by Hustache?, cut; "263" handwr. on pink paper. To all three pins we add "Geraeus / aureomicans / Hustache 1924" handwr. on red paper. "MACN-En / 9653 [through 9657]" print., reverse. Ex CBC.

Note: Tucumán was not mentioned in the original description. Only a spec. from Corrientes and one from Bolivia were mentioned. 
boliviensis [Listroderes] Hustache 1924: 160. Chaco de Santiago del Estero Prov.: Rio Salado, febrero, type in HUS. Bolivia: Izozo VIII.1917; Cuatro Ojos IX.1917.

boliviensis [Ctenomyophila]: Hustache 1926: 179, 185. Type from Chaco de Santiago; La Rioja Prov. ex Bruch (sic); Bolivia: Izozo, etc. (sic).

boliviensis [Hormops]: Wibmer \& O'Brien 1986: 121.

Three spec. pinned, "Rep. Argentina / A. Breyer" print., a color stripe indicative of La Rioja Prov.; "Typus" ["Cotypus", "Cotypus"] print. on pale green paper. One of them also "Ctenomyophila / boliviensis / Hust." handwr. by Bruch, red frame; "enomyophil / Boliviensis m." handwr. by Hustache?, cut. To all three we add "Listroderes / boliviensis / Hustache 1924 / Syntypus?" handwr. on red paper. "MACN-En / 9449 [9450, 9451]" print., reverse. Ex CBC.

Note: The type condition is puzzling: La Rioja was not mentioned in the original description, but it was included by Hustache (1926) from that province as from Bruch (not from Breyer).

bosqi (sub Bosqui) [Conotrachelus] Hustache 1924: 186. Buenos Aires, several spec., type in HUS from Bosq. Bolivia: Trinidad X.1917, one spec.

bosqi (sub Bosqi) [Conotrachelus]: Hustache 1926: 186. In a key; Buenos Aires from Bosq \& Lizer y Trelles, type in HUS; Buenos Aires Prov. from Bruch; Corrientes Prov.: San Roque from Bosq; Jujuy Prov. from Bruch. Bolivia: Trinidad. bosqi [Pheloconus]: Wibmer \& O'Brien 1986: 171. C. Bosqui Hustache 1924 is a lapsus, corrected to Bosqi by Hustache (1926).

Two spec. on cards, on separate pins, "Rep. Argentina / Prov. Jujuy / 190 / C. Bruch" print., black frame; "Cotypus" print. [handwr. by Bruch] on pale green paper; "Conotrachelus / Bosqi Hust." handwr. by Bruch, red [green] frame; the 1st one also "12" handwr. on pink paper, "luxatus à decrire", "onotrac / Bosqi" handwr., both cut; the 2nd one also "19271" handwr. (in register book same data). Ex CBC.

Notes: The type condition of these spec. is puzzling: Jujuy was not mentioned in the original description, and was included in 1926. Six synt. from Buenos Aires Prov. are recorded in the main list, above.

brachyderoides [Adioristus] Hustache 1926: 188. Tucumán Prov.: Famaillá, leg. Weiser one spec.

brachyderoides [Scotoeborus]: Kuschel 1949: 31. brachyderoides [Cylydrorhinus]: Wibmer \& O'Brien 1986: 103.

One spec. pinned, "Rep. Argentina / Prov. Tucuman / 27.II.1906 / C. Bruch" print., date handwr., black frame; "Foto", "Typus" print. on pale green paper; "Adioristus / brachyderoides / Hust." handwr. by Bruch, red frame; "Listroderes / brachyderoides *" handwr. by Hustache?, cut. “MACN-En / 9563" print., reverse, Ex CBC.

Notes: The type condition is puzzling, as only one spec. from Tucumán Prov.: Famaillá was originally mentioned, recorded as holot. at the main list, above.

bruchi (sub Bruchi) [Coeliodes, sub Coeliodes] Hustache 1926: 257, pl. IV: f. 3. Tucumán Prov. 10.VII.1900 from Bruch.

bruchi (sub Bruchi) [Hypocoeliodes]: Hustache 1947a: 444 .

One spec. on a card, "Alta Gracia. La Granja / Sierras de Córdoba / C. Bruch" print., "Typus" print. on pale green paper; we add "Coeliodes / bruchi / Hustache 1926" handwr. on red paper. Ex CBC.

Note: Córdoba was not mentioned in the original description.

bruchi (sub Bruchi) [Lichnus] Hustache 1951b: 2-3. Buenos Aires Prov. 25.X.1904, Bruch n ${ }^{\circ} 122$, = Prolichnus Bruchi in litteris.

bipunctatus [Lichnus] (Blanchard 1843): Kuschel 1955: 274. = L. bruchi.

bipunctatus [Lichnus] (Blanchard 1843): Wibmer \& O'Brien 1986: 340. = L. bruchi Hustache, $=$ L. bruchi var. rufipennis.

One synt.? on a card, "Rep. Argentina / Prov. Buenos Aires / 1.1897 / C. Bruch" print., date handwr., black frame; "Typus" handwr. by Bruch on pale green paper; "Prolichnus / Bruchi / Hust." handwr. by Bruch, green frame. We add "Lichnus / Bruchi / Hustache 1951 / Syntypus?" handwr. on red paper. Ex CBC.

Note: The collecting date differs from that of the original description, rending the type condition doubtful. The spec. is labeled only by Bruch, not by Hustache.

brunneopilosus [Baris] Hustache 1924: 120121. Bolivia: Yacuiva XII.1917, type; Santa Cruz IX.1917.

brunneopilosa [Rhytidobaris]: Hustache 1938b: 29.

balteata [Methyorrhina] (Casey 1922): Kuschel 1983: 40. = R. brunneopilosa

One spec. on a card, "Rep. Argentina / Prov. 
Tucuman /10.II.1907 / C.Bruch" print. date handwr., black frame; "Typus" handwr. by Bruch on pale green paper; "Rhytidobaris / brunneopilosa / Hust." handwr. by Bruch, without frame; "Rhytidobaris / brunneopilosa m." handwr. by Hustache?, cut; "264a" handwr. on pink paper. We add "Baris / brunneopilosus / Hustache 1924 / Syntypus?" handwr. on red paper. "MACN-En / 10019" print., reverse. Ex CBC.

Note: Tucumán was not mentioned in the original description.

brunneus [Pantomorus] Hustache 1947b: 123124. Catamarca Prov.: Caspinchanga (sic, pro Caspinchango) 7.III.1921, from Bruch $\mathrm{n}^{\circ} 43$, type in HUS.

One spec. on a card, "Bañado /Tucumán V.921" handwr. by Bruch; "Typus" print. on pale green paper; "Mimographus / brunneus / Hust." handwr. by Bruch, red frame; "Mimographus / brunneus*" handwr. by Hustache?, cut; "Pantomorus / brunneus / Hustache / det. Lanteri 90" handwr on white paper. "MACN-En / 10073" print., reverse. Ex CBC.

Note: Tucumán was not mentioned in the original description.

ciliata [Baris] Hustache 1951a: 71. Paraguay: Asunción, types; Hohenau leg. Jacob. Uruguay: Montevideo leg. Lecocq 1937; San José: Sierra Mahoma 28.XII.1936; Rio Negro: San Javier leg. Denier 18.XI.1930.

beckeri [Baris] Bondar 1949: Kuschel 1983: 35. $=$ B. ciliata.

One spec. on a pointed card, "Lages (Brasilien) / H. Fruhstorfer leg. I-III-87. vend." print.; "Typus" handwr. by Bruch on pale green paper; "Baris / ciliata / Hust." handwr. by Bruch, without frame; "Baris / ciliata m." handwr. by Hustache?, cut. "MACN-En / 10562" print. reverse. $E x$ CBC.

Note: Brasil was not mentioned in the original description.

curta [Ctenomyophila] Hustache 1926: 179, 184. Córdoba Prov.: Alta Gracia XII.1920, one spec.; Jujuy, one spec.

[curtus] [Hormops]: Kuschel 1964: 423.

curtus [Hormops]: Wibmer \& O'Brien 1986: 121.

One spec. on a card, "Fuerte Quemado / Catam. Wolters" handwr. by Bruch ; "Typus" print. on pale green paper; "Ctenomyophila / curta / Hust." handwr. by Bruch, red frame; " 226 " handwr. on pink paper. "MACN-En / 9464" print., reverse. Ex CBC.
Note: Catamarca was not mentioned in the original description.

curvirostris [Parasomenes] Hustache 1939: 115. In a key. Misiones Prov., from Bruch.

One spec. pinned, "Rep. Argentina / Prov. Buenos Aires / 190_ / C. Bruch" print., black frame; "Typus" handwr. by Bruch on pale green paper; "Parasomenes / curvirostris / Hust." handwr. by Bruch, without frame; "Parasomenes / curvirostris m." handwr. by Hustache?, cut. "MACN-En / 9658" print., reverse. Ex CBC.

Note: Buenos Aires was not mentioned in the original publication.

elegantulus [Platyomus] Hustache 1923: 290-291. Chaco de Santiago [del Estero], type in HUS. Tucumán: Colmenar, in HUS. Bolivia: Lagunillas XI.1917, one spec.

One spec. on a card, "Chiquimil / Catam. Wolters" handwr. by Bruch; "Cotypus" handwr. by Bruch on pale green paper; "Platyomus / elegantulus / Hust." handwr. by Bruch, green frame; "19291" handwr. (in register book "Alta Gracia (Córdoba)", registration error?). "MACNEn / 10018" print., reverse. Ex CBC.

Note: Catamarca was not mentioned in the original publication. One synt.? from Tucumán is recorded at the main list, above.

fuscum [Prionobrachium] Hustache 1924: 175-176. Chaco de Santiago del Estero Prov.: Rio Salado, type in HUS. Bolivia: Izozo VIII.1917; Cuatro Ojos IX:1917. Brasil: Minas Gerais State: Matsinhos, from Gounelle, in HUS.

One spec. on a card, "Rep. Argentina / Prov. Buenos Aires / 190_ / C. Bruch" print., black frame; "Typus" print. on pale green paper; "171" handwr. on pink paper; "Prionobrachium / fuscum / Hust." handwr. by Bruch, red frame; "Prionobrachium / fuscum m." handwr. by Hustache?, cut; "171" handwr. on pink paper. One spec. on a card, "Rep. Argentina / Prov. / Tucuman 190_/ C. Bruch" print., black frame; "Typus", "Foto" print. on pale green paper; we add "Prionobrachium / fuscum / Hustache 1924" handwr. on red paper. "MACN-En / 10137 [10138]" print., reverse. Ex CBC.

Note: Neither Buenos Aires nor Tucumán were mentioned in the original publication.

griseomaculatus [Archopactus cyphoides var.] Hustache 1923: 280. Chaco de Santiago [del Estero] Prov.: Rio Salado, in HUS.

griseomaculatus [Naupactus cyphoides var.]: Dalla Torre, Emden \& Emden 1936: 18. 
cyphoides [Naupactus] Heller 1921: Ward, O’Brien, O'Brien, Foster \& Huddleston 1977: 24. = griseomaculatus.

cyphoides [Naupactus] Heller 1921: Wibmer \& O'Brien 1986: 59. = griseomaculatus, = prasinus Hustache 1947 = viridimarginalis Hustache 1947.

One spec. pinned, "Rep. Argentina / Prov. Catamarca / 21.1.1920 / C. Bruch” print., date handwr., black frame; "Cotypus" print. on pale green paper; "Archopactus

(Naupactus) / griseomaculatus / Hust." handwr. by Bruch, red frame; "griseomaculatus / var. m." handwr. by Hustache?, cut. "MACN-En /10056" print., reverse. Ex CBC.

Notes: Catamarca was not mentioned in the original publication. One synt. from Santiago del Estero Prov. is recorded in the main list, above.

niger var. [Chalcodermus] Hustache 1924: 189190. Bolivia; Santa Cruz IX.1917, two spec., probably 9 .

One spec. on a card, "Alta Gracia. La Granja / Sierras de Córdoba / II.922. C. Bruch leg." print., date handwr.; "Typus" print. on pale green paper; " 176 " handwr. on pink paper; "Chalcodermus / niger / Hust. var." handwr. by Bruch, red frame; "alcodern / niger m. var." handwr. by Hustache?, cut. "MACN-En / 10141" print., reverse. Ex CBC.

Notes: Córdoba was not originally mentioned. The word "var." on the labels suggests that an unnamed variety was meant.

nitens [Alhypera] Hustache 1926: 173-174. Chaco Prov., leg. Bruch X.1895.

breyeri [Argentinorhynchus] Brèthes 1910: Kuschel 1950: 112. = A. nitens Hustache.

One spec. on a card, "Rep. Argentina / Gob. Misiones / 12.1896 / C. Bruch" print., date handwr., black frame; "Cotypus" print. on pale green paper. We add "Alhypera / nitens / Hustache 1926" handwr. on red paper. "MACN-En / 9525" print., reverse. Ex CBC.

Notes: Córdoba was not originally mentioned. A synt. from Chaco Prov. is recorded in the main list, above.

persimilis [Brachygeraeus] Hustache 1950b: 3-4. "Gran Chaco"; [Buenos Aires Prov.]: Tandil, from Bruch n 262.

[persimilis] [Eucalus]: Bondar 1950: 472.

[persimilis] [Linogeraeus]: Kuschel 1983: 38.

Four spec. on two double cards $(2+2)$ on one pin, "Rep. Argentina / Prov. Tucuman / 6.III.1900
/ C. Bruch" print., date handwr., black frame; "Typus" handwr. by Bruch on pale green paper; "Brachygeraeus / persimilis / Hust." handwr. by Bruch, green frame. "MACN-En / 9665 [9666, 9667, 9668]” print., reverse. Ex CBC.

Note: Tucumán was not originally mentioned.

pilosipes [Rhyssomatus] Heller 1921: 35. Córdoba Prov., ô, o l leg. Bruch; Mendoza Prov.: Santa Rosa, leg. Jensen-Haarup.

One spec. pinned, "Rep. Argentina / Prov. Tucuman / 15.I.1904 / C. Bruch" print., date handwr., black frame; "Typus" print. on pale green paper. We add "Rhyssomatus / pilosipes / Heller 1921". "MACN-En / 10155" print., reverse. $E x \mathbf{C B C}$.

Note: Tucumán was not mentioned. A synt. from Córdoba is recorded at the main list, above.

planicollis [Platyomus] Hustache 1926: 165166, pl. I : f. 4. Catamarca Prov. leg. Bruch 21.I.1910.

One spec. pinned, "Sgo. del Estero / Rio Salado / Wagner col.," print.; "Foto", "Typus" print. on pale green paper; "Platyomus / planicollis / Hust." handwr. by Bruch, red frame. One spec. pinned, "Rep. Argentina / Prov. Corrientes / 190 / C. Bruch" print., black frame; "Typus" print. on pale green paper. We add "Platyomus / planicollis / Hustache 1926". "MACN-En / 9985 [9986]" print., reverse. Ex CBC.

Note: Neither Santiago del Estero nor Corrientes Prov. were mentioned in the original description.

postmaculatus [Geraeus] Hustache 1924: 99 100. French Guyana: Nouveau Chantier (type in HUS), Roches de Kourou (id.). Argentina: Buenos Aires, one spec. from Lizer y Trelles, in HUS. Bolivia: Cuatro Ojos IX.1917, one spec. posticemaculatus [Centrinaspis]: Hustache 1938a: 105.

postmaculatus [Linogeraeus]: Kuschel 1983: 39.

One spec. on a card, "Rep. Argentina / Prov. Buenos Aires / 10.X.1906 / C. Bruch" print.date handwr., black frame; "Typus" handwr. by Bruch on pale green paper; "271" handwr. on pink paper; "Centrinaspis / postmaculatus / Hust." handwr. by Bruch, green frame. We add "Geraeus / postmaculatus / Hustache 1924 / Syntypus?" handwr. on red paper. "MACN-En / 9661" print., reverse. Ex CBC.

Note: The type condition is doubtful, as it is said that the spec. from Buenos Aires is in HUS. The spec. is labeled only by Bruch, not by Hustache. 
punctatellas (sic, propunctatella) [Liobaridia] Hustache 1951a: 10 (in a key; not in text; punctatellas is a lapsus pro punctatella). Formosa Prov.

punctatella [Liobaridia]: Wibmer \& O'Brien 1986: 294.

One spec. pinned, "Estancia la Noria / Rio San Javier / Santa Fe. Argentina / G.E. Bryant. 23.XII.1911" print., date handwr., a green horizontal stripe ; "G. Bryant / Coll. 1919-147" print.; "Typus" handwr. by Bruch on pale green paper; "Heterosternus / punctatellus / Hust." handwr. by Bruch, without frame; "Heterosternus / punctatellus m." handwr. by Hustache?, cut. We add "Liobaridia / punctatella / Hustache 1951" handwr. on red paper. "MACN-En / 9618" print., reverse. $E x$ CBC.

Note: Sante Fe was not mentioned in the original description.

rubromaculatus [Chalcodermus] Hustache 1924: 267-268. Brasil: Jatahy, type in HUS. [Bolivia:] Cuatro Ojos IX.1917.

Two spec. on cards, on separate pins, "Rep. Argentina / Prov. Buenos Aires / 190_ / C. Bruch" print., black frame; "Typus" print. on pale green paper; "172" handwr. on pink paper. One of them also "Chalcodermus / rubromaculatus / Hust." handwr. by Bruch, red frame; "Chalcodermus / rubromaculatus" handwr. bu Hustache?, cut, remainings of "m.". The other one also "Foto" print. on pale green paper. We add "Chalcodermus / rubromaculatus / Hustache 1924" handwr. on red paper. "MACN-En / 10035 [10036]" print., reverse. Ex CBC.

Note: Buenos Aires Prov. was not mentioned in the original description.

seminebulosus [Conotrachelus] Brèthes 1910: 219-220. Bolivia

histrio [Conotrachelus] Boheman 1859: Hustache 1926: 254. = C. seminebulosus.

coelebs [Conotrachelus] Boheman 1837: Wibmer \& O’Brien 1986: $157=$ C. histrio.

One spec. on a card, remounted, "Misiones" handwr. on green paper, reverse white; "Repreparo / M. Viana / I-1960" handwr.; "10240" handwr.; "Conotrachelus / seminebulosus / Brèthes" handwr. by Brèthes on whitish paper. "MACNEn / 10362" print., reverse. Ex JB.

Note: Misiones was not mentioned in the original description. A synt.? without locality label is recorded in the main list, above.

sharpi [Pseudopantomorus] Heller 1921: 22-23, f. 1. Tucumán Prov., from Bruch. Paraguay, in coll. Faust.

sharpi [Parapantomorus]: Dalla Torre, Emden \& Emden 1936: 25.

fluctuosus [Parapantomorus] (Boheman 1840): Kuschel 1955: 280. Nov. combin., = P. sharpi Heller.

One spec. on a card, "Rep. Argentina / Gob. Misiones / 190_ / C. Bruch" print., black frame. One spec. on a card, "Rep. Argentina / Gob. Chaco / 190 / C. Bruch" print., black frame. Both: "Typus" print. on pale green paper. We add "Pseudopantomorus / sharpi / Heller 1921" handwr. on red paper. "MACN-En / 10070 [10071]" print. reverse. Ex CBC.

Notes: Misiones and Chaco were not mentioned in the original publication. A synt. from Tucumán is recorded at the main list, above.

subbimaculatus [Pantomorus] Hustache 1947b: 129-130. Argentina [probably error for Uruguay]: Cerro, from Tremoleras, in HUS. Corrientes Prov.: San Roque leg. Bosq II.1920, Entre Ríos Prov.; Córdoba Prov.: Calamuchita leg. Viana.

marginalis [Naupactus] Boheman 1833: Kuschel 1955: 279.

cinerosus [Naupactus] Boheman 1833: Wibmer \& O'Brien 1986: 59. = marginalis Boheman, = parsevali (Costa Lima), = inimicus (Marshall), = roseomicans (Hustache), = subbimaculatus (Hustache), = var. ursius (Hustache).

Four spec. on cards, on one pin, "Prov. de Buenos Aires / J. Bosq col." print.; "Paratype" handwr. on pale green paper; "37767" handwr. (in register book same data); "Pantoplanes / subbimaculatus / Hust." handwr. by Bruch, black frame. "MACN-En / 10074 [10075, 10076, 10077]" print., reverse. Ex CBC.

Notes: Buenos Aires was not mentioned in the original publication. The spec. is labeled only by Bruch, not by Hustache.

tucumanica [Centrinaspis] Hustache 1950b: 19. Tucumán Prov., from Bruch $n^{\circ} 259$.

tucumanicus [Geraeus]: Kuschel 1983: 37.

One spec. on a card, "Alta Gracia. La Granja / Sierras de Córdoba / 24.IX.24. C. Bruch leg." print., date handwr.; "Typus" handwr. by Bruch on pale green paper; "259" handwr. on pink paper; "Centrinaspis / tucumanensis (sic) / Hust." handwr. by Bruch, green frame. We add "Centrinaspis / tucumanica / Hustache 1950" handwr. on red paper. "MACN-En / 9660" print., reverse. Ex $\mathbf{C B C}$. 
Note: Córdoba was not mentioned in the original publication. The spec. is labeled only by Bruch, not by Hustache.

variegatus [Euxenus] Hustache 1924: 271-272. Chaco de Santiago del Estero Prov.: Rio Salado, type in HUS. Bolivia: Santa Cruz IX.1917, one spec.

cubae [Faustinus] (Boheman 1844): Monte 1944: 295, f. 5. = tabaci (Marshall 1925), = variegatus (Hustache 1924).

Two spec. on cards, on separate pins, "Rep. Argentina / Prov. Buenos Aires / 20.I.1904 [XI.1897] / C. Bruch" print., dates handwr., black frames; "Typus" print. [handwr. by Bruch] on pale green paper; "Euxenus / variegatus / Hust." handwr. by Bruch, red [green] frame. The 1st one also " 157 " handwr. on pink paper; "Euxenus / variegat" handwr. by Hustache?, cut. The 2nd one also "19274" handwr. (in register same data). "MACN-En / 10091 [10092]" print., reverse. Ex CBC.

Note: Buenos Aires was not mentioned in the original publication.

vicina [Liobaridia] Hustache 1951b: 10, 11. Tucumán Prov., from Bruch $n^{\circ} 253$; Salta Prov. Paraguay: Hohenau.

One spec. on a card, "La Ciénaga (Belén) / Catamarca 192_ / Weiser Wolters leg." print.; "Fototypus" handwr. by Bruch on pale green paper. "MACN-En / 9631" print., reverse. Ex CBC. Notes: Catamarca was not mentioned in the original publication. Three synt. from Salta and Tucumán Prov. are recorded in the main list, above.

vicinus [Listroderes] Hustache 1929: 199, 200, pl. II: f. 1. Catamarca Prov.: Fuerte Quemado, from Bruch.

difficilis [Listroderes] Germain 1895: Name resurrected by G. Kuschel in Wibmer \& O’Brien 1986: 113.

Four spec. on cards, on separate pins. The 1st, 2nd, \& 4th spec. "Fives Lille / S. Fé Weiser" print. The 1st, 2nd, \& 3rd spec. "Typus" print. on pale green paper. The 1st, 3rd, \& 4th spec. "Listroderes / vicinus / Hust." handwr. by Bruch, on red [red, green] paper. The 1st spec. also "istr / vicinus m." handwr. by Hustache?, cut. The 2nd spec. also "Foto" print. on pale green paper. The 3rd spec. also "Listroderes / vicinus m." handwr. by Hustache?, cut; "112" handwr. on pink paper; "Molino Puesto / Catam. Weiser" handwr. by Bruch. The 4th spec. also "Cotypus" handwr. by Bruch on pale green paper; "19285" handwr. (in register book same data); "148" handwr. on pink paper. "MACN-En / 10165 [10166, 10167, 10168]" print., reverse. Ex CBC

Note: Only the 3rd spec. was collected in Catamarca Prov., but in a different locality. The whole series is puzzling, and probably none should be accepted as a type.

viduatus [Brachygeraeus] Hustache 1950b: 6 . Brasil: Jatahy.

viduatus [Linogeraeus]: Kuschel 1983: 44.

One spec. on a card, "Rep. Argentina / Prov. Tucuman / 3.1897 / C. Bruch" print., date handwr., black frame; "Typus" handwr. by Bruch on pale green paper; "261" handwr. on pink paper; "Brachygeraeus / viduatus / Hust." handwr. by Bruch, green frame. "MACN-En / 9659" print., reverse. Ex CBC.

Note: Tucumán was not mentioned in the original publication.

villosulus [Adioristus] Hustache 1926: 186, 189. Jujuy Prov.: Santa Catalina, from Bruch.

villosulus [Scotoeborus]: Kuschel 1949: 34-35, pl. II: f. 7. = A. griseus Germain 1911 nomen nudum.

villosulus [Cylydrorhinus]: Wibmer \& O'Brien 1986: 107. = griseus (Germain) nom. $n u d .$, = villosulus var. unicolor Hustache.

One spec. on a card, "Laguna Blanca / Catam. Weiser" handwr. by Bruch ; "Typus" print. on pale green paper; "Adioristus / villosulus / Hust." handwr. by Bruch, red frame; "Adioristu / villosulus" handwr. by Hustache?, cut. "MACN-En / 9507" print., reverse. Ex CBC.

Note: Catamarca was not mentioned in the original publication. Two synt. from Jujuy Prov. are recorded in the main list, above.

\section{ACKNOWLEDGEMENTS}

We express our appreciation to the specialists that have assisted us with bibliography referred to Scolytidae, Platypodidae, Anthribidae and Brentidae, Dr. Robert Anderson from the Canadian Museum of Nature and Dr. José Ricardo Mermudes from the University of Rio de Janeiro, Brasil. For help with management after Aurora of the data base in the Entomology Division of the Museum, we are sincerely indebted to Natalia Cuadra. Last, but not least, to Dr. Cristina L. Scioscia for a very conscious and precise revision of the last draft of this paper. We also thank the Consejo Nacional de Investigaciones Científicas y Técnicas (CONICET) for its continuous financial support. 


\section{BIBLIOGRAPHY}

Alonso-Zarazaga, M.A. \& Lyal, C.H.C. 1999. A world catalog of families and genera of Curculionoidea (Insecta : Coleoptera) (excluding Scolytidae and Platypodidae). Entomopraxis SCP, Barcelona, Spain, $315 p$.

Béguin-Billecocq, L. 1909. Apions nouveaux de la République Argentine (Col. Curculionidae). Annales de la Société Entomologique de France 78: 449-464.

Béguin-Billecocq, L. 1911. Contribution à la faune des coléoptères de l'Amérique du Sud. Diagnoses d'espèces nouvelles d'Apionidae (Col. Curculionidae). Bulletin de la Société Entomologique de France 1911: 131-133.

Blackwelder, R.E. 1947. Checklist of the coleopterous insects of Mexico, Central America, the West Indies, and South America. Part 5. United States National Museum Bulletin 185 (5): 765-921.

Boheman, C.H. 1837. In Schönherr, C.J. Genera et Species Curculionidum 4 (1): 1-600.

Boheman, C.H. 1859 Coleoptera. Species novas descripsit. Kongliga Svenska Fregatten Eugenies Resa Omkring Jorden... Zoologi I. Insecta 113-218.

Bondar, G. 1941a. Notas entomologicas da Baía. VII. Revista de Entomologia, Rio de Janeiro 12 (1/2): 268-303, f. 1-18.

Bondar, G. 1941b. Notas entomologicas da Baía. VIII. Revista de Entomologia, Rio de Janeiro 12 (3): 427-470, f. 1-31.

Bondar, G. 1942a. Notas entomologicas da Baía. IX. Revista de Entomologia, Rio de Janeiro 13 (1/2): 1-39, f. 1-7. [Hustache \& Bondar in...].

Bondar, G. 1942b. Notas entomologicas da Baía. X. Revista de Entomologia, Rio de Janeiro 13 (3): 225-274.

Bondar, G. 1943. Notas entomologicas da Baía. XI. Revista de Entomologia, Rio de Janeiro 14 (1/2): 33-84.

Bondar, G. 1949. Notas entomologicas da Baía. XXI. Revista de Entomologia, Rio de Janeiro 20 (1-3): 173-228, f. 1-15.

Bondar, G. 1950. Notas entomologicas da Baía. XXII. Revista de Entomologia, Rio de Janeiro 21 (3): 449-480.

Bosq, J. 1943. Segunda lista de coleópteros de la República Argentina, dañinos a la agricultura. Ingeniería Agronómica 4 (18-22): 7-80.

Brèthes, J. 1909. Dos nuevos Platypus (Col.) argentinos. Anales del Museo Nacional, Buenos Aires 17 [3 3 serie 10): 225-227, f. 1-3.

Brèthes, J. 1910. Coleópteros argentinos y bolivianos. Anales de la Sociedad Científica Argentina 69: 205-227.

Brèthes, J. 1913a. Description d'un Pandeleteius (Coléoptères Curculionides) nouveau de Buenos Aires. Boletín de la Sociedad Physis, Buenos Aires 1 (4): 192-193.

Brèthes, J. 1913b. Description d'un nouveau curculionide du Chili. Revista Chilena de Historia Natural 17 (1/2): 39-40, f. 3 [Boletín del Museo Nacional,
Chile 5 (1): 138-139, f. 3] [On both: "Abril 1913" on the cover].

Brèthes, J. 1919a. Quelques insectes de Manaos, avec description d'un Platypus (Col.) et d'un Neomesostenus (Hym.) nouveaux. Revista Chilena de Historia Natural 23 (1/2): 26-28.

Brèthes, J. 1919b. Deux coléoptères chiliens nouveaux. Anales de Zoología Aplicada (Agricola, Médica, Veterinaria), Santiago, Chile 6 (1): 26-29, f. 3, 4.

Brèthes, J. 1920. Insectes du Pérou. Anales de la Sociedad Científica Argentina 89: 27-54.

Brèthes, J. 1921. Notas coleopterológicas. Revista de la Facultad de Agronomía, La Plata 14 (3) (3a época): 163-169, f. 1-4.

Brèthes, J. 1922. Descripción de varios coleópteros de Buenos Aires. Anales de la Sociedad Científica Argentina 94: 263-305.

Brèthes, J. 1923. Description d'un nouveau genre et une nouvelle espèce d'Ipidae du Chili. Revista Chilena de Historia Natural 25, 1921: 433-435, f. 37., Brèthes, J. 1925. Coléoptères et Diptères chiliens. Revista Chilena de Historia Natural 29: 198-208.

Brèthes, J. 1926. Descripción de un gorgojo que ataca la zanahoria: "Aulametopiellus dauci", n. gen. n. sp. Physis, Buenos Aires 8 (30): 414-416.

Bright, D.E. \& Skidmore, R.E. 1997. A Catalog of Scolytidae and Platypodidae (Coleoptera), Supplement 1 (1990-1994). NRC Research Press, Ottawa., Canada.

Bright, D.E. \& Skidmore, R.E. 2002. A catalog of Scolytidae and Platypodidae (Coleoptera). Supplement 2 (1995-1999). NRC Research Press, Ottawa, Canada 523 p.

Bruch, C. 1912. Oxycorynus Helleri un nuvo gorgojo argentino. Anales del Museo Nacional de Historia Natural, Buenos Aires 23: 265-267, $1 \mathrm{f}$.

Bruch, C. 1914. Un nuevo ípido (escolítido) de Chile (Phloeotribus Porteri n. spec.). Anales de Zoología Aplicada. Santiago, Chile 1: 25-27, pl. II: f. A-E.

Bruch, C. 1915. Catálogo sistemático de los coleópteros de la República Argentina. Revista del Museo de La Plata 19: 401-441.

Bruch, C. 1916. Un nuevo gorgojo del "Prosopanche" (Oxycorynus parvulus Bruch). Revista del Museo de La Plata 23: 231-233, 4 f.

Bruch, C. 1940. Misceláneas Entomológicas III. Notas del Museo de La Plata, Zool. 5 (35): 111-122.

Buchanan, L.L. 1939. The species of Pantomorus of America North of Mexico. United States Department of Agriculture, Miscellaneous Publications (341): 1-39 p., $5 \mathrm{f}$.

Buchanan, L.L. 1941. In Cortés, P.R.: Acerca del género Pantomorus Schoenh. (Col. Curculionidae) en Chile. Agricultura Técnica, Chile 1 (1): 61-63.

Buchanan, L.L. 1942. Four new species of white fringed beetles (subgenus Graphognathus) from the southwestern part of the United States (Coleoptera: Curculionidae). Bulletin of the Brooklyn Entomological Society 87 (3): 107-110.

Buchanan, L.L. 1947. A correction and two new races in Graphognathus (white fringed beetles) (Coleoptera: Curculionidae). Journal of the 
Washington Academy of Sciences 37 (1): 19-22, f.

Burke H.R. 1963. New species of Texas weevils, with notes on others (Coleoptera, Curculionidae). Southwestern Naturalist 8 (3): 162-172.

Burmeister, H. 1879. Briefliche Mittheilungen. 4. Die patagonischen Arten der Gattung Cylindrorrhinus. Stettiner Entomologische Zeitung 40: 203-209.

Casey, T.L. 1922. Studies in the rhynchophorous subfamily Barinae of the Brazilian fauna. Memoirs on the Coleoptera 10: 1-520.

Champion, G.C. 1904. Insecta (Curculionidae: Curculioninae). Biologia Centrali Americana, Coleoptera 4 (4): 313-440.

Clark, W. E. 1978. The weevil genus Sibinia Germar: natural history, taxonomy, phylogeny and zoogeography, with revision of the New World species (Coleoptera: Curculionidae). Quaestiones Entomologicae 14 (2): 91-387.

Clark, W.E., Burke, H. \& Mc Kay, F. 2007. The weevil genus Achia Champion (Coleoptera: Curculionidae): new species associated with Urvillea (Sapindaceae) and new Serjania host plant records for A. ancile Burke and A. affinis Hustache. The Coleopterists Bulletin 61(4): 542-550.

Csiki, E. 1934. Curculionidae: Cleoninae. Coleopterorum Catalogus 28 (134): 1-151.

Csiki, E. 1936. Curculionidae: Rhynchophorinae. Coleopterorum Catalogus 30 (149): 3-104.

Dalla Torre, K.W.von, Emden, M. van \& Emden, F. van 1936. Curculionidae: Brachyderinae I. Coleopterorum Catalogus 27 (147): 1-132.

del Río, M.G., Lanteri, A.A. \& Suárez, S.M. 2005. Types of Scolytidae and Platypodidae (Coleoptera: Curculionidae) housed at the Museo de La Plata entomological collection. Revista del Museo de La Plata 46: 1-11.

Emden, F. I. van 1952. On the taxonomy of the Rhynchophora larvae: Adelognatha and Alophinae (Insecta: Coleoptera). Proceedings of the Zoological Society of London 122 (3): 651-795, illus.

Emden, M. van \& Een, F. van 1939. Curculionidae: Brachyderinae III. Coleopterorum Catalogus 27 (164): 197-327.

Günther, K. \& Zumpt, P. 1933. Curculionidae: Tanymecinae. Coleopterorum Catalogus 27 (131): 1-131.

Haedo Rossi, J.A. 1959. Nuevas entidades de "Brenthidae" para la fauna de la República de Bolivia (Brenthidae, Coleoptera). Acta Zoologica Lilloana 17: 95-118, pl. I-II.

Haedo Rossi, J.A. \& Viana, M. 1956. Un nuevo género y especie de Anthribidae neotrópico (Coleop.). Revista de la Sociedad Entomológica Argentina 19 (1/2): 21-26, f. 1, 2.

Hagedorn, M. 1909. Diagnosen bisher unbeschriebener Borkenkäfer (Col.). Zweite Serie, erste Hälfte. Deutsche Entomologische Zeitschrift 1909: 733746, f. II: 30-38, 40, 41.

Hagedorn, M. 1910. Diagnosen bisher unbeschriebener Borkenkäfer (Col,). Zweite Serie, zweite Hälfte. Deutsche Entomologische Zeitschrift 1910: 1-13, f. $39,42-47$.

Heller, K.M. 1906. Neue Rüsselkäfer aus Central- und
Südamerika. Stettiner Entomologische Zeitung 67 (1): 3-50, pl. I: f. 1-12.

Heller, K.M. 1911. Übersicht der Oxycorinus-Arten. Wiener Entomologische Zeitung 30 (1): 5-8, f. 1, 2.

Heller, K.M. 1920. Ein neuer, im Neste der Kammratte (Ctenomys) lebender Rüsselkäfer aus Argentinien. Entomologische Blätter 16 (10/12): 239-241, $1 \mathrm{f}$.

Heller, K.M. 1921. Nuevos curculiónidos de la Argentina. Anales de la Socidad Científica Argentina 91: 1935, f. 1-12.

Howden, A. 1976. Pandeleteius from Venezuela and Colombia (Curculionidae: Brachyderinae: Tanymecini). Memoirs of the American Entomological Institute (24): i, 1-310, f.

Howden, A. 1982. Revision of the New World genus Hadromeropsis Pierce (Coleoptera: Curculionidae: Tanymecini). Contributions of the American Entomological Institute 19 (6): i-iii, 1-180.

Hustache, A. 1923/1924. Curculionides de l'exploration Lizer - Delétang au Chaco Bolivien. Anales de la Sociedad Científica Argentina 96, 1923: 279-300; Suite 1. 97, 1924: 156-191, 267-284; Suite 2. 98, 1924: 91-124.

Hustache, A. 1926. Contribution à l'étude des Curculionides de la République Argentine (première note). Anales del Museo Nacional de Historia Natural, Buenos Aires 34 (Entomol. 140): 155-261, pl. I-V.

Hustache, A. 1929. Nouveaux curculionides de l'Amérique du Sud. Revista de la Sociedad Entomológica Argentina 2 (10): 227-232.

Hustache, A. 1932. Un Apion nouveau et quelques remarques au sujet de divers Curculionides [Col.]. Bulletin de la Société Entomologique de France 37: 136-137

Hustache, A. 1936a. Curculionidae: Cryptorhynchinae. Coleopterorum Catalogus 39 (151): 1-317.

Hustache, A. 1936b. Nouveaux Sphénophorides SudAméricains (Curculionidae). Bulletin et Annales de la Société Entomologique de Belgique 76: 87-114.

Hustache, A. 1938a Compsus sud-américains (Col. Curculionidae). Bulletin et Annales de la Société Entomologique de Belgique 78: 67 -118.

Hustache, A. 1938b. Curculionides de l'Argentine et des régions limitrophes. Revista de la Sociedad Entomológica Argentina 10 (1): 3-17.

Hustache, A. 1938c. Curculionides nouveaux de l'Amérique méridionale, que se trouvent dans le Deutsches Entomologisches Institut. 1. - 3. Arbeiten über Morphologische und Taxonomische Entomologie 5: 174-184, 265-288, 334-348.

Hustache, A. 1938d. Curculionidae: Barinae. Coleopterorum Catalogus 30 (163): 1-219.

Hustache, A. 1939. Curculionides nouveaux de l'Argentine et autres régions Sud-Américaines. Anales de la Sociedad Científica Argentina 128: 38-46 [sic pro 64], 99-124, f. 1-5.

Hustache, A. 1940a. Curculionides nouveaux de l'Argentine et autres régions Sud-Américaines (Deuxième note). Anales de la Sociedad Científica Argentina 129: 112-144.

Hustache, A. 1940b. Curculionides nouveaux de l'Argentine et autres pays limitrophes de la co- 
llection P. Denier (Première note). Anales de la Sociedad Científica Argentina 129: 272-284.

Hustache, A. 1947a. Nouvelle contribution à l'étude des Ceutorrhynchininae (Col. Curc.). Revista de Entomologia, Rio de Janeiro 17 (3): 444-451.

Hustache, A. 1947b. Naupactini de l'Argentine et des régions limitrophes (Col. Curculion.). Revista de la Sociedad Entomológica Argentina 13: 3-146.

Hustache, A. 1950a. Nouveaux Baridinae sud américains. Première partie. Ambatini, Peridinetini, Pantotelini, Cyrionychina et Optanini. Boletim do Museu Nacional, Rio de Janeiro, nova série Zool. (95), 1949: 1-55.

Hustache, A. 1950b. Nouveaux Baridinae sud américains. Troisième partie. Centrinina. Boletim do Museu Nacional, Rio de Janeiro, nova série Zool. (97), 1949: 1-143.

Hustache, A. 1951a. Nouveaux Baridinae sud américains. Deuxième partie. Barina. Boletim do Museu Nacional, Rio de Janeiro, nova série Zool. (96): $1-78$.

Hustache, A. 1951b. Nouveaux Barinae sud-américains, 4ème partie. Madopterina - Lytherina -Leptoschoina-Eutoxina-Madarina-Barymerina. Boletim do Museu Nacional, Rio de Janeiro, nova série Zool. (102): 1-78.

Hustache, A. \& Bondar, G. 1942 in Bondar, G. Notas entomologicas da Baía. IX. Revista de Entomologia, Rio de Janeiro 13 (1/2): 1-39.

Hustache, A. \& Bruch, C. 1936. Descripción y notas biológicas de un curculiónido mirmecófilo (Col. Curcul.). Revista de Entomologia, Rio de Janeiro 6 (3/4): 332-338, f. 1-12, pl. 3: f. 1-5.

Jacob, H. 1937. Die Curculioniden der Kolonie Hohenau (Beobachtungen und Sammelergebnisse). Entomologische Jahrbücher 46: 150-158.

Jordan, K. 1904. American Anthribidae. Novitates Zoologicae 11: 242-309.

Kissinger, D.G. 2005. A new species of Coelocephalapion Wagner (Coleoptera: Curculionoidea: Apionidae: Apioninae) from Argentina and Chile associated with the genus Prosopis L. (Fabaceae). The Coleopterists Bulletin 59 (4): 493-500.

Kuschel, G. 1943. Un "gorgojo acuático del arroz" argentino, Lissorhoptrus bosqi n. sp. (Col. Curculionidae). Notas del Museo de La Plata, Zool. 8 (71): 305-315, f. 1, 1 pl.

Kuschel, G. 1945a. Aportes entomológicos I. (Curculionidae). Anales de la Sociedad Científica Argentina 139: 120-136.

Kuschel, G. 1945b. Aportes entomológicos (II). (Coleop. Curculionidae). Los Cratosomus argentinos. Revista de la Sociedad Entomológica Argentina 12 (5): 261-381, f. 1-12, photos 1-12 [on pl.XXXIXXXIII].

Kuschel, G. 1949. Los "Curculionidae" del extremo norte de Chile (Coleoptera, Curcul. op. $6^{\circ}$ )..Acta Zoologica Lilloana 8: 5-54, f. 1-8, pl. I-III.

Kuschel, G. 1950a. Nuevas sinonimias, revalidaciones y combinaciones ( $9^{\circ}$ aporte a Col. Curculionidae). Agricultura Técnica, Chile 10 (1): 10-21.

Kuschel, G. 1950b. I. Nuevos Curculionidae de Bolivia y Perú. II. Notas a algunas especies de Brèthes (Ap.
7 de Col. Curcul.). Revista del Museo de La Plata Zool. 6 (38): 69-116, f. 1-15, pl. I-III.

Kuschel, G. 1950c. Nuevos Brachyderinae y Magdalinae chilenos (Coleoptera Curculionidae) (Aporte $5^{\circ}$ ). Arthropoda, Buenos Aires 1 (2/4): 181-195, f. 1-5, photos 1-9.

Kuschel, G. 1952a. Revisión de Lissorhoptrus Leconte y géneros vecinos de América (Ap. 11 de Coleoptera Curculionidae). Revista Chilena de Entomología 1, 1951: 23-74, f. 1-46.

Kuschel, G. 1952b. Los Curculionidae de la cordillera chileno-argentina (1 ${ }^{\mathrm{a}}$ parte) (Aporte $13 \mathrm{de}$ Coleoptera Curculionidae). Revista Chilena de Entomología 2: 229-279.

Kuschel, G. 1952c. Cylindrorhininae aus dem Britischen Museum (Col. Curculionidae. 8. Beitrag). Annals and Magazine of Natural History, $12^{\text {th }}$ Ser., 5 (50): 121-137, f. 1-5.

Kuschel, G. 1955. Nuevas sinonimias y anotaciones sobre Curculionoidea (Coleoptera). Revista Chilena de Entomología 4: 261-312.

Kuschel, G. 1958a. Nuevos Cylydrorhininae de la Patagonia (Col. Curculionoidea. Aporte 18). Investigaciones Zoológicas Chilenas 4: 231-252, f. $1-23$.

Kuschel, G. 1958b. Neotropische Rüsselkäfer aus dem Museum G. Frey (Col. Curculionidae). Entomologische Arbeiten aus dem Museum G. Frey 9 (3): 750-798, f. 1-71.

Kuschel, G. 1959a. Beiträge zur Kenntnis der Curculioniden von Venezuela und Trinidad-Insel (1. Lieferung). Entomologische Arbeiten aus dem Museum G. Frey 10 (2): 478-514, f. 1-59.

Kuschel, G. 1959b. Nemonychidae, Belidae y Oxycorinidae de la fauna chilena, con algunas consideraciones biogeográficas (Coleoptera Curculionoidea, aporte 28). Investigaciones Zoológicas Chilenas 5: 229-271, f. 1-96.

Kuschel, G. 1964. Insects of Campbell Island. Coleoptera: Curculionidae of the subantarctic islands of New Zealand. Pacific Insects Monographs 7: 416-493.

Kuschel, G. 1971. Entomology of the Aucklands and other islands south of New Zealand: Coleoptera: Curculionidae. Pacific Insects Monographs 27: 225-259, f.

Kuschel, G. 1983. New synonymies and combinations of Baridinae from the Neotropic and Nearctic Regions (Coleoptera: Curculionidae). The Coleopterists Bulletin 37 (1): 34-44.

Lanteri, A.A. 1982. Estudio taxonómico del género Wagneriella Hustache (Coleoptera: Curculionidae). Revista de la Sociedad Entomológica Argentina 41 (1-4): 61-64.

Lanteri, A.A. 1990a. Revisión sistemática del género Priocyphus Hustache 1939 y creación de los géneros Priocyphopsis y Lamprocyphopsis (Coleoptera, Curculionidae). Revista Brasileira de Entomologia 34 (2): 403-422, f. 1-89, tab. 1.

Lanteri, A.A. 1990b. Revisión sistemática del género Cyrtomon Schönherr (Coleoptera: Curculionidae). Revista Brasileira de Entomologia 34 (2): 387-402.

Lanteri, A.A. 1995. Systematic revision of Ericydeus 
Pascoe (Coleoptera: Curculionidae). Entomologica scandinavica 26 (4): 393-424.

Lanteri, A.A. \& Díaz, N.B. 1994. Systematic study and cladistic analysis of the genus Aramigus Horn (Coleoptera: Curculionidae). Transactions of the American Entomological Society 120 (2): 113-144.

Lanteri, A.A. \& Loiácono, M.S. 1990. Systematic study of the Pantomorus viridisquamosus species group (Coleoptera: Curculionidae). Insecta Mundi 4 (1/4): 1-10.

Lanteri, A.A. \& Marvaldi, A.E. 1995. Graphognathus Buchanan, a new synonym of Naupactus Dejean, and systematics of the $N$. leucoloma species group (Coleoptera: Curculionidae). The Coleopterists Bulletin 49 (3): 206-228.

Lanteri, A.A. \& O’Brien, C.W. 1990. Taxonomic revision and cladistic analysis of Atrichonotus Buchanan (Coleoptera: Curculionidae). Transactions of the American Entomological Society 116 (3): 697-725.

Lanteri, A.A. , Suárez, S.M. \& del Río, M.G. 2003. Types of Curculionidae (Coleoptera: Curculionoidea) housed at the Museo de La Plata entomological collection. Revista de la Sociedad Entomológica Argentina 62 (3-4): 35-48.

Lawrence, J.F. \& Newton, A.F. 1995. Families and subfamilies of Coleoptera (with selected genera, notes, references and data on family-group names). In: Papaluk, J. \& Šlipiński, S.A. (ed.) Biology, Phylogeny, and Classification of Coleoptera. Papers Celebrating the $80^{\text {th }}$ Birthday of Roy A. Crowson 2: 779-1092.

Mantilleri, A. \& Sforzi, A. 2006. Review of the Neotropical species of the genus Stereodermus Lacordaire, 1866 (Coleoptera: Brentidae, Stereodermini). Zootaxa 1207: 1-64.

Marshall, G.[A.K.] 1933. New Neotropical Curculionidae (Col.). Stylops 2 (3): 59-69, f.

Marshall, G.[A.K.] 1946. Taxonomic notes on Curculionidae (Col.). Annals and Magazine of Natural History $11^{\text {th }}$ ser.,13: 93-98.

Marvaldi A.E., Sequeira A.S., O’Brien C.W. \& Farrell B.D. 2002. Molecular and morphological phylogenetics of weevils (Coleoptera, Curculionidea): Do niche shifts accompany diversification? Systematic Biology 1: 761-785.

Mermudes, J.R.M. 2002. Systaltocerus platyrhinus Labram \& Imhoff, 1840: redescrição e considerações sobre a sinonímia com Homalorhamphus vestitus Haedo Rossi \& Viana, 1957 (Coleoptera, Anthribidae, Anthribinae). Revista Brasileira de Entomologia 46 (4): 579-590, f. 1-60.

Mermudes, J.R.M. \& Napp, D.S. 2004. Duas novas espécies de Ptychoderes Schoenherr (Coleoptera, Anthribidae, Anthribinae, Ptychoderini). Revista Brasileira de Entomologia 48 (1): 27-30.

Monte, O. 1944. Notas sôbre curculionídeos. Revista Brasileira de Biologia 4 (3): 289-296, f.

Morrone, J.J. 1994a. Systematics, Cladistics and Biogeography of the Andean genera Macrostyphlus, Adioristidius, Puranius, and Amathynetoides new genus (Coleoptera: Curculionidae). American Museum Novitates (3104): 1-63.

Morrone, J.J. 1994b. Systematics of the Patagonian genus Acrostomus Kuschel (Coleoptera: Curculionidae). Annals of the Entomological Society of America 87 (4): 403-411.

Morrone, J.J. 1995. Nomenclatural changes in Patagonian Belidae and Curculionidae (Coleoptera: Curculionoidea). Neotropica, La Plata 40, 1994 (103/104): 95.

Morrone, J.J. \& Anderson, R. 1995. The Falklandius genus group: Cladistic analysis with description of new taxa (Coleoptera: Curculionidae: Rhytirrhinini). American Museum Novitates (3121): 1-14.

Morrone, J.J. \& Lanteri, A.A. 1991. Ubicación sistemática y variación intraespecífica de Pantomorus rui$z i$ (Brèthes) (Coleoptera: Curculionidae). Revista de la Sociedad Entomológica Argentina 49, 1990: 17-26, pl.1-4.

Morrone, J.J. \& Loiacono, M. 1992. Revision of the genus Rhigopsidius Heller (Insecta: Coleoptera: Curculionidae: Rhytirrhininae). Entomologische Abhandlungen, Staatliches Museum für Tierkunde in Dresden 54 (7): 129-139.

Morrone, J.J. \& Loiacono, M. 1994. Los ejemplares tipo de Curculionidae sensu lato (Coleoptera: Curculionoidea) depositados en la colección del Museo de La Plata. Revista del Museo de La Plata, Serie Técnica y Didáctica 15: 1-41.

Morrone, J.J. Marvaldi, A.E. \& O’Brien, C.W. 1995. Lixellus Leconte, a new synonym of Listronotus Jekel (Coleoptera: Curculionidae). Entomological News 106 (3): 108-112.

Neave, S.A. 1939/1955. Nomenclator Zoologicus. A list of the names of genera and subgenera from the tenth edition of Linnaeus 1758 to the end of 1935, vol. 1, 2, 1939, vol. 3, 4, 1940, vol. 5, 1950.

O'Brien, C.W. 1976. A taxonomic revision of the New World subaquatic genus Neochetina (Coleoptera: Curculionidae: Bagoini). Annals of the Entomological Society of America 69 (2): 165-174.

O'Brien, C.W.1979. Hyperodes new synonym of Listronotus, with a checklist of Latin American species (Cylindrorhininae: Curculionidae: Coleoptera). Southwestern Entomologist 4 (4): 265-268.

O'Brien, C.W.1984. Revision of the Neotropical weevil genus Philonis (Cryptorhynchinae: Curculionidae: Coleoptera). Southwestern Entomologist 9 (2): 232-239, f. 1-8.

O'Brien, C.W.\& Wibmer, G.J. 1982. Annotated checklist of the weevils (Curculionidae sensu lato) of North America, Central America, and the West Indies. Memoirs of the American Entomological Institute (34): IX + 382 p.

O’Brien, C.W.\& Wibmer, G.J. 1984. Annotated checklist of the weevils (Curculionidae sensu lato) of North America, Central America, and the West Indies. Supplenent 1. Southwestern Entomologist 9 (3): 286-307.

Papp, C.S. 1978. Catalog of the genus Conotrachelus with references to the literature and deposition of the type material (Coleoptera: Curculionidae). Calif. Dep. Food \& Agric., Div. Plant. Ind., Lab. Serv., Entomol. 1978: 1-83.

Rheinheimer, J. 2004. Illustrierter Katalog und Bibliographie der Anthribidae der Welt (Insecta: 
Coleoptera). Mittelungen des Entomologischen Vereins, Stuttgart 39: 3-242.

Rodríguez, D. 2007-2012. Aurora. Aplicación para el manejo de datos de colecciones biológicas. Museo Argentino de Ciencias Naturales "Bernardino Rivadavia", Consejo Nacional de Investigaciones Científicas y Técnicas, CONICET.

Schedl, K.E. 1939. Fauna argentinensis. III. 70ª comunicación a la morfología y sistemática de Scolytidae (Col.). Notas del Museo de La Plata, Zool. 4 (28): 407-412, f. 1-5.

Schenkling,S. \& Marshall, G.A.K. 1931. Curculionidae: Eremninae, Leptopinae, Tanyrrynchinae, Cylindrorrhininae, Thecesterninae (Suppl.), Rhytirrhininae (Suppl.), Rhyparosominae (Suppl.). Coleopterorum Catalogus 28 (114): 1-162.

Sforzi, A. \& Bartolozzi, L. 2004 (eds.). Brentidae of the World (Coleoptera: Curculionoidea). Museo Regionale di Scienze Naturali- Regione Piamonte, Torino. Monographie XXXIX.

Valentine, B.D. 1960. The genera of the weevil family Anthribidae North of Mexico (Coleoptera). Transactions of the Entomological Society of America 86 (1): 41-85.

Vaurie, P. 1953. The Gregorio Bondar types of South American weevils (Coleoptera, Curculionidae). American Museum Novitates (1615): 1-39.

Vaurie, P. 1970. Weevils of the tribe Sipalini (Coleoptera, Curculionidae, Rhynchophorinae). Part 2. The genera Mesocordylus and Orthognathus. American Museum Novitates (2441): 1-78, f. 1-77.

Vaurie, P.1978. Revision of the genus Sphenophorus in South America. American Museum Novitates (2656): 1-30, f. 1-23. tab. 1.

Viana, M.J. 1937. Lista de insectos de la isla Martín García. Revista de la Sociedad Entomológica Argentina 9: 101-109.

Viana, M.J. 1949. Nuevas especies argentinas de Tachygonus y catálogo de la subfamilia Tachygoninae (Coleop., Curculionidae). Revista del Instituto Nacional de Investigación de las Ciencias Naturales, Ciencias Zoológicas 1 (3): 67-93, f. 1-4, pl. I: f. $1-4$

Viana, M.J. 1951. Nuevas especies de cuculiónidos argentinos. Subfamilia Amalactinae (Coleop. Curculionidae). Comunicaciones del Instituto Nacional de Investigación de las Ciencias Naturales, Ciencias Zoológicas 2 (3): 29-45, f. 1-7, pl. I-III.

Viana, M.J. 1954. Nuevas especies de Tachygoninae neotropicales (Coleop. Curcul. Tachygoninae). Comunicaciones del Instituto Nacional de Investigación de las Ciencias Naturales, Ciencias Zoológicas 2 (11): 151-177, f. 1-14.

Viana, M.J. 1957. Dos especies nuevas de Entimus Germar (Coleop. Curculionidae, Leptopinae). Boletín del Museo Argentino de Ciencias Naturales (11): $13 \mathrm{p}$.

Viana, M.J. 1960. El género Atenistes Pascoe, en la RepúblicaArgentina, con una especie nueva (Coleop. Curculionidae, Myrmecinae). Actas y Trabajos del $1^{\circ}$ Congreso Sudamericano de Zoología, La Plata 1959 3: 245-258, f. 1-6.

Viana, M.J. 1968. Entimus excelsus sp. nov., de Brasil
(Coleopt., Curculionidae, Leptopinae) Neotropica, La Plata 24 (45): 103-106, f. 1-3. Serie Extra, Museo Argentino de Ciencias Naturales (58): 103106, f. 1-3.

Voss, E. 1954. Curculionidae (Col.). 100. Beitrag zur Kenntnis der Curculioniden. In Titschack (Ed.). Beiträge zur Fauna Perus 4:193-376, 1 tab.

Voss, E. 1957. Archimetrioxena electrica Voss und ihre rezenten Formenkreisen (Col. Curc.). 142. Beitrag zur Kenntnis der Curculioniden. Deutsche Entomologische Zeitschrift Neue Folge 4 (1/2): 95102, f. 1-8.

Wagner, H. 1912. Beitrag zur Kenntnis der ApionFauna Central und Südamerikas. Beschreibungen neuer Arten, nebst synonymischen Bemerkungen. Mémoires de la Société Entomologique de Belgique 19, 1911: 1-32, pl. I-III.

Ward, C.R., O’Brien, C.W., O'Brien, L., Foster, D.E. \& Huddleston, E.W. 1977. Annotated checklist of new world insects associated with Prosopis (Mesquite). Technical Bulletin, Texas Technical University (1557): $67 \mathrm{p}$.

Warner, R.E. 1975. New synonyms, key, and distribution of Graphognathus, white-fringed beetles (Coleoptera: Curculionidae) in North America. United States Department of Agriculture, Coop. Econ. Ins. Report 25 (44): 855-860.

Wibmer, G.J. 1989. Revision of the weevil genus Tyloderma Say (Col.: Curculionidae) in Mexico, Central America, South America, and the West Indies. Evolutionary Monographs (11): 1-118, f. $1-143$.

Wibmer, G.J. \& O'Brien, C.W. 1986. Annotated checklist of the weevils (Curculionidae sensu lato) of South America (Coleoptera: Curculionoidea). Memoirs of the American Entomological Institute (39): I-XVI + 1-563.

Wibmer, G.J \& O'Brien, C.W. 1989a. Revision of the Neotropical genus Neohydronomus Hustache (Coleoptera: Curculionidae). The Coleopterists Bulletin 43 (3): 291-304.

Wibmer, G.J. \& O'Brien, C.W. 1989b Additions and corrections to annotated checklist of the weevils of North America, Central America, and the West Indies, and of South America. Southwestern Entomologist Suppl. 13: 1-49.

Wolfrum, P. 1931. Neue Anthribiden. 4. Beitrag zur Kenntnis der Anthribiden. Entomologische Blätter 27: 70-76.

Wood, S.L. 1993. Revision of the genera of Platypodidae (Coleoptera). Great Basin Naturalist 53: 259-281.

Wood, S.L \& Bright, D.E. 1992. A catalog of Scolytidae and Platypodidae (Coleoptera). Part 2: Taxonomic Index Volumes A \& B. Great Basin Naturalist, Memoirs 13: $1553 \mathrm{p}$.

Zimmerman, E.C. 1994. Australian weevils. I. Orthoceri. Anthribidae to Attelabidae. The primitive weevils. CSIRO, Australia.

Recibido: 27-X-2012 Aceptado: 6-VIII-2013 